\title{
Decoding the Pre-Eruptive Magnetic Field Configurations of Coronal Mass Ejections
}

\author{
S. Patsourakos ${ }^{1} \cdot$ A. Vourlidas ${ }^{2} \cdot$ T.
} Török ${ }^{3}$. B. Kliem ${ }^{4}$. S. K. Antiochos ${ }^{5}$. V. Archontis ${ }^{6}$. G. Aulanier ${ }^{7}$. X. Cheng ${ }^{8}$. G. Chintzoglou ${ }^{9}$. M.K. Georgoulis ${ }^{10}$. L.M. Green ${ }^{11}$. J. E. Leake ${ }^{5}$. R. Moore ${ }^{12}$. A. Nindos ${ }^{1}$. P. Syntelis ${ }^{6}$. S. L. Yardley ${ }^{6}$. V. Yurchyshyn ${ }^{13} \cdot$ J. Zhang ${ }^{14}$

Received: date / Accepted: date

\begin{abstract}
A clear understanding of the nature of the pre-eruptive magnetic field configurations of Coronal Mass Ejections (CMEs) is required for understanding and eventually predicting solar eruptions. Only two, but seemingly disparate, magnetic configurations are considered viable; namely, sheared magnetic arcades (SMA) and magnetic flux ropes (MFR). They can form via three physical mechanisms (flux emergence, flux cancellation, helicity condensation). Whether the CME culprit is an SMA or an MFR, however, has been strongly debated for thirty years. We formed an International Space Science Institute (ISSI) team to address and resolve this issue and report the outcome here. We review the status of the field across modeling and observations, identify the open and closed issues, compile lists of SMA and MFR observables to be tested against observations and outline research activities to close the gaps in our current understanding. We propose that the combination of multi-viewpoint multi-thermal coronal observations and multiheight vector magnetic field measurements is the optimal approach for resolving

\footnotetext{
${ }^{1}$ Department of Physics, University of Ioaninna, Ioaninna, Greece

2 Johns Hopkins University Applied Physics Laboratory, Laurel, MD, USA

. ${ }^{3}$ Predictive Science Inc., 9990 Mesa Rim Road, Suite 170, San Diego, CA 92121, USA

. 4 Institute of Physics and Astronomy, University of Potsdam, 14476 Potsdam, Germany

. ${ }^{5}$ Goddard Space Flight Center, Greenbelt, MD, USA

6 School of Mathematics and Statistics, University of St. Andrews, St. Andrews, UK

. 7 LESIA, Observatoire de Paris, Université PSL, CNRS, Sorbonne Université, Université de Paris, 5 place Jules Janssen, 92195 Meudon, France

. 8 School of Astronomy and Space Science, Nanjing University, Nanjing 210093, People's Republic of China

. 9 Lockheed Martin Solar and Astrophysics Lab, Palo Alto, California, USA

. 10 Research Center Astronomy and Applied Mathematics, Academy of Athens, Athens, Greece

${ }^{11}$ University College London, Mullard Space Science Laboratory, Holmbury St. Mary, Dorking, Surrey RH5 6NT, UK

. ${ }^{2}$ NASA/Marshall Space Flight Center, Huntsville, Alabama, USA

. ${ }^{13}$ Big Bear Solar Observatory, New Jersey Institute of Technology, Big Bear City, California, USA
}

${ }^{14}$ Department of Physics and Astronomy, George Mason University, Fairfax, VA, USA
\end{abstract}


the issue conclusively. We demonstrate the approach using MHD simulations and synthetic coronal images.

Our key conclusion is that the differentiation of pre-eruptive configurations in terms of SMAs and MFRs seems artificial. Both observations and modeling can be made consistent if the pre-eruptive configuration exists in a hybrid state that is continuously evolving from an SMA to an MFR. Thus, the 'dominant' nature of a given configuration will largely depend on its evolutionary stage (SMA-like early-on, MFR-like near the eruption).

Keywords Plasmas - Sun: activity - Sun: corona - Sun: magnetic fields - Sun: Coronal Mass Ejections · Sun: Space Weather

\section{Introduction}

Coronal Mass Ejections (CMEs) are large-scale expulsions of magnetized coronal plasma into the heliosphere. They represent a key energy release process in the solar corona, and a major driver of space weather. Despite the observations and cataloguing of the properties of thousands of events in the last 40 years, the formation and nature of their pre-eruptive magnetic configurations are still eluding us. Which magnetic configurations are most prone to destabilize and form a CME and how they arise remain hotly debated questions.

The pre-eruptive magnetic configuration of CMEs has been modeled either as a sheared magnetic arcade (SMA) or as a magnetic flux rope (MFR). In some theoretical/numerical models, the pre-eruptive configuration is an MFR (e.g., Chen, 1989, Amari et al, 2000, Török and Kliem, 2005) and in some the pre-eruptive configuration is a SMA (Moore and Roumeliotis, 1992, Antiochos et al, 1999).

Both of these structures are capable of containing dipped, sheared field lines above a polarity inversion line (PIL), and so are candidates for the magnetic structure of a filament channel (defined in Section 1.1). Strong PILs are characterized by the concentration of most of the shear (i.e., non-potentiality) of the magnetic field, and hence free magnetic energy therein.

An important point is that all models predict that the CME will contain an MFR after eruption. Indeed, MFR-like structures are often detected in CME EUV (e.g. Dere et al, 1997, Zhang et al, 2012, Vourlidas, 2014) and coronagraphic observations, (e.g., Vourlidas et al, 2013, 2017) and in in-situ measurements (e.g., Burlaga et al, 1981; Nieves-Chinchilla et al, 2018). Since the ejected structure is an MFR, white-light coronagraphic or in-situ observations should not generally be expected to provide a direct way to determine whether the pre-eruptive configuration was an SMA or an MFR.

Although there seem to exist only two possible pre-eruptive magnetic geometries (SMA and MFR), several physical mechanisms (e.g., shearing, flux emergence, flux cancellation, helicity condensation) can give rise to either of them. There is a vast literature on the subject but there is no consensus on where and when given mechanisms may be relevant, if they need to operate alone or in tandem, and whether they are also the cause or the trigger of the subsequent eruption. Obtaining a clearer picture of what is the pre-eruptive configuration and how it forms will have important implications for the physical understanding of CMEs and the origin and evolution of CME-prolific active regions (ARs). Our improved 
understanding will help us in turn to better evaluate the eruptivity of a given $\mathrm{AR}$ and hence improve our predictive and forecasting abilities for space weather purposes (e.g., Vourlidas et al, 2019).

Despite significant advances in modeling, theory, and observational capabilities, the resolution of these issues is hampered by a number of factors: (i) limitations of observations (e.g., lack of routine magnetic-field observations above the photosphere, line-of-sight confusion in imaging), (ii) limitations of models (e.g., idealized boundary conditions, high numerical diffusion in the MHD codes), (iii) inconsistent application of terms and definitions (defining an MFR in the observations or the onset time of an eruption, for example) to observations leading to ambiguous conclusions, and perhaps most importantly (iv), MFRs and SMAs can be nearly indistinguishable in either field line plots or observations (e.g., filament threads).

Addressing these issues was the motivation behind the formation of an ISSI team tasked with 'decoding the pre-eruptive configuration of CMEs'. The team met twice with three objectives: (1) debate the formation and configuration of filament channels, which comprise the observational manifestations of MFRs and SMAs; (2) identify the open and closed issues; and (3) propose a path forward, for both modeling and observations, to resolve these issues. The results of our discussions form the core of this manuscript. We do not attempt to provide a comprehensive review of CME initiation and formation processes since there exist several recent excellent reviews on the matter (Chen, 2011; Cheng et al, 2017; Manchester et al, 2017, Green et al, 2018; Archontis and Syntelis, 2019; Georgoulis et al, 2019, Liu, 2020). We focus on three key questions: (1) what constitutes an MFR or SMA?; (2) how can MFRs/SMAs be identified in the solar atmosphere?; and (3) how do they form? We compile a set of recommendations for solving these outstanding issues in the future, whether via modeling or improved observational data.

This paper is organized as follows. First, we provide definitions for the most important terms, such as MFR, SMA, Filament Channel, etc., to provide a clear baseline for the discussion. In Section 2 we review the three dominant mechanisms for filament channel formation: flux emergence, flux cancellation, and helicity condensation. In Section 3 , we discuss the modeling expectations and observational signatures for SMAs and MFRs. In Section 4. we summarize our findings and provide a table containing MFR/SMA observables that could be checked against observations. In Section 5 , we present recommendations for resolving the nature of the pre-eruptive configuration and its formation and in the Appendix $\mathrm{A}$ we demonstrate an appropriate methodology using MHD simulations and synthetic coronal images.

\subsection{Basic Definitions}

This section contains definitions for the key terms discussed throughout the manuscript. The definitions are guided by practical considerations, i.e, to help interpret observations and models.

Pre-Eruptive Condition: This is the key term for our discussion, yet it is hard to define precisely. Eruptions occur over a wide range of time scales and exhibit a variety of signatures at the surface and in the lower atmosphere. For example, some eruptions precede, others follow, the soft X-ray (SXR) flare onset 


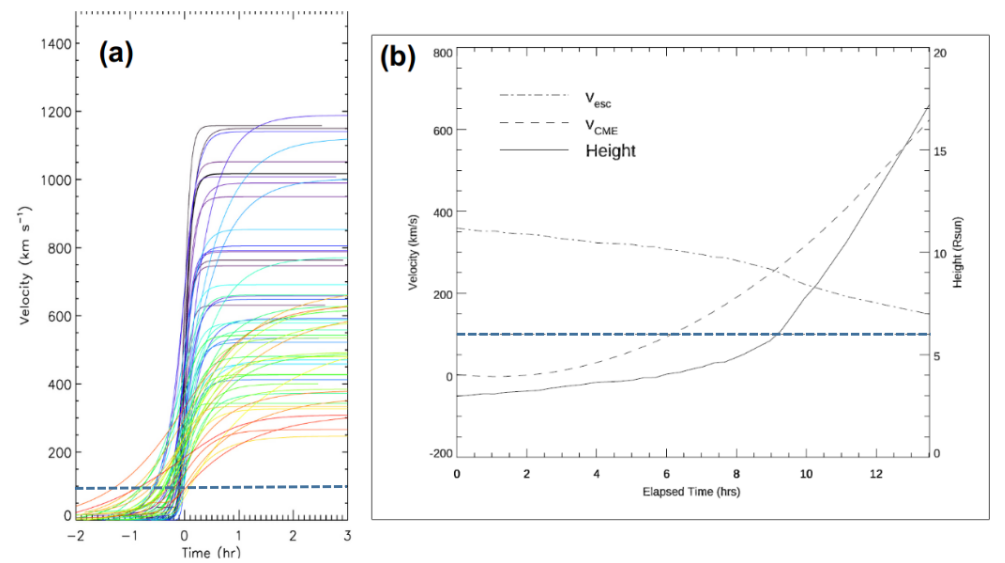

Fig. 1 Panel (a): superposed epoch analysis of the velocity-time profiles of 42 CMEs associated with eruptive flares. The key time of the profiles (i.e., time $=0$ ) corresponds to the time of maximum acceleration for each CME. Modified from Zhu et al (2020) CAAS. Reproduced with permission. Panel (b): velocity-time profile of a streamer blowout CME (dashes). Adapted from Vourlidas et al (2002). In both panels, the horizontal dashed blue lines correspond to a speed of $100 \mathrm{~km} / \mathrm{s}$

and some are not accompanied by flares at all. The timing of, or rather the determination of whether an eruption is under way, is crucial in assessing the physical processes responsible for it (ideal or non-ideal, for example). Since there is no widely-accepted measure or definition of the start of an eruption, we put forth one as follows. It is logical to expect that a CME will occur when the speed of the rising structure exceeds a considerable fraction of the local Alfvén speed. At that point, the rising structure can no longer be considered in a state of a quasi-static rise caused by, for instance, slow photospheric motions. This quantity is, however, very difficult to assess observationally or via simulations since the coronal magnetic environment is insufficiently known. Instead, we take a more practical, and conservative, approach and consider a high enough speed to ensure that a CME will occur across the range of CME source regions. We posit that $100 \mathrm{~km} \mathrm{~s}^{-1}$ is a practical limit based on observations of both flare-related and streamer-blowout CMEs (e.g., Figure 1). Hence, we propose the following definition for the preeruptive condition: The pre-eruption phase ends when the speed of the rising (and eventually, released) magnetic structure exceeds $100 \mathrm{~km} \mathrm{~s}^{-1}$.

Polarity Inversion Line (PIL): The line separating areas of opposite magnetic polarity on the Sun.

Filament channel (FC): Filament channels are the upper atmospheric counterparts of PILs. They correspond to regions where the magnetic field is largely aligned with the photospheric PIL. FCs are identified in the chromosphere by the orientation of chromospheric fibrils. When partially filled with cool and dense plasma, FCs manifest themselves as filaments or prominences in chromospheric 

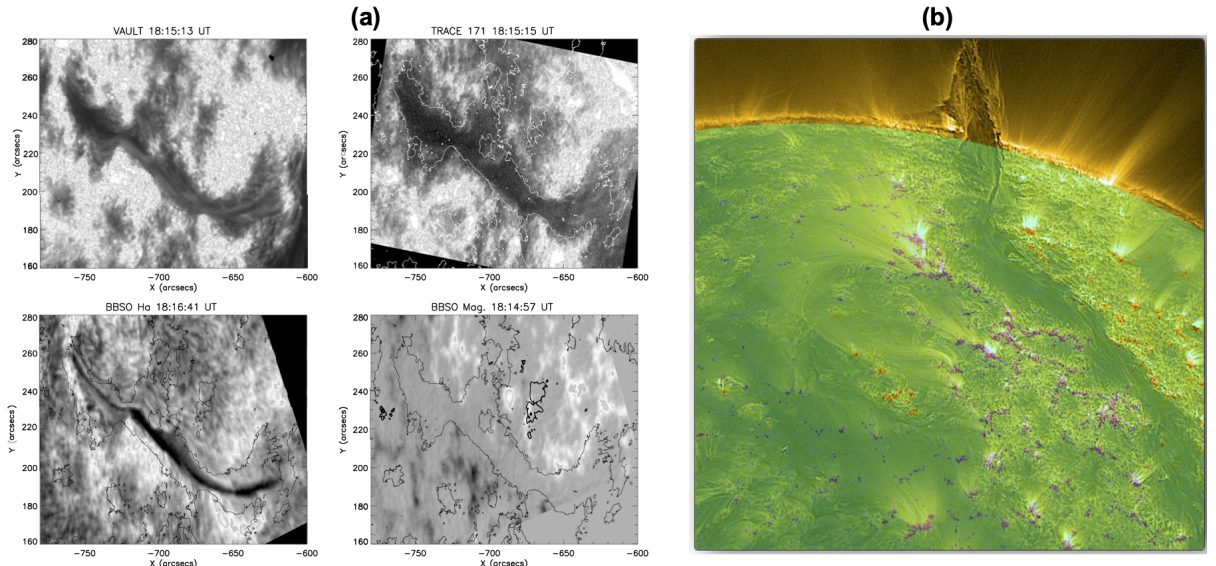

Fig. 2 Panel (a): A filament channel with prominence material observed in Lyman- $\alpha$ (top left), $171 \AA$ (top right), $\mathrm{H} \alpha$ (bottom left) and photospheric magnetic field (bottom right). The contours delineate the extent of the Lyman- $\alpha$ prominence. From Vourlidas et al (2010). C)2009, Springer Nature. Panel (b): Composite of AIA $171 \AA$ (gold) and HMI magnetogram (green overlay with positive flux in red and negative flux in blue) of a spectacular filament channel containing a filament/prominence.

and coronal observations e.g, see Figure 2 .

Sheared Magnetic Arcade (SMA): A set of field lines (or flux bundles) that cross a PIL with an orientation deviating from the local normal to the PIL (see Section 3.1. An SMA does not have an axis field line about which its flux, or an inner part of its flux, twists from end to end but it can nevertheless contain a small amount of twisted flux. In a strongly sheared arcade, the orientation of the field lines closely follows the PIL. In an SMA, the sheared flux is much larger than the twisted flux. SMA models range from simple, pseudo-2.5D cases that contain only arched field lines of the same orientation (Figure 3(a)) to more complex, fully 3D, cases that contain S-shaped and dipped field lines (Figure 3(b)). In this review, we mainly discuss these more realistic, complex SMAs.

Magnetic Flux Rope (MFR): A twisted flux tube where the majority of the interior field lines wind about a common axial field line along the length of the tube. This is an expanded definition of the textbook MFR (Priest, 2014) to account for the complexities encountered in current models and their comparisons with observations, which we discuss in detail in the rest of the paper. MFRs are characterized by the presence of a magnetic axis, a current channel, and twist extending over the full length of the magnetic axis (Figure 3(c)). We classify MFRs based on the twist number $N$ (their end-to-end number of turns) as: 1) weakly twisted $(N<1) ; 2)$ moderately twisted $(N \approx(1-2))$; 3$)$ highly twisted $(N>2)$. MFRs do not necessarily possess uniform twist, or twist peaking in the vicinity of the axis (Figure 2(c)) - field lines near the axis can have minimal twist (Figure $2(\mathrm{~d})$ ). However the defining MFR characteristic is that the twisted flux is much larger than the sheared flux. The axis of MFRs relevant to eruptions follows the PIL relatively closely. For an arched MFR to possess dips, one must additionally 
(a)

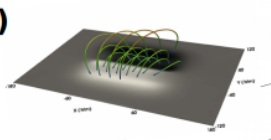

(c)

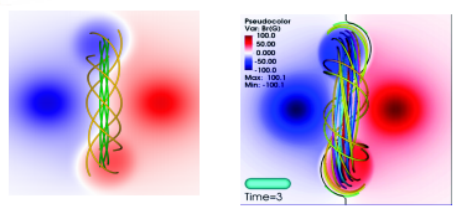

(b)

(d)

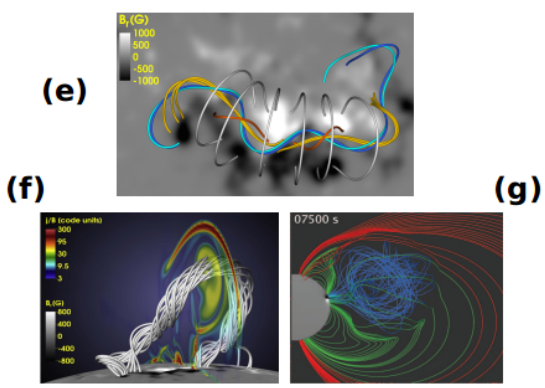

Fig. 3 Pre-eruptive (panels a-e) and eruptive configurations (panels f-g) from MHD simulations. Magnetic fields lines are shown in all panels. Panel (a): 2.5D SMA. Modified from Zhou et al (2018). Panel (b): 3D SMA. Modified from DeVore and Antiochos (2000). Panel (c): MFR with twisted field lines concentrated in the core of the configuration. Modified from Titov et al (2014). Panel (d): MFR with twisted field lines concentrated in the periphery of the configuration (i.e., hollow-core configuration). Modified from Titov et al (2014). Panel (e): hybrid configuration. Modified from Török et al (2018a). Panel (f): eruptive MFR from a simulation employing a pre-eruptive MFR that has evolved from the [hybrid] configuration shown in panel (e) . Modified from Török et al (2018a). Panel (g): eruptive MFR from a simulation employing a pre-eruptive SMA. Modified from Lynch et al (2008). All figures are (CAAS and reproduced with permission.

require at least $\sim 1$ end-to-end turn (see Section 3.2 for details). Examples of eruptive MFRs resulting from pre-eruptive MFRs or SMAs are given in panels (f) and (g) of Figure 3 respectively.

SMA-MFR Hybrid: A magnetic configuration that contains both sheared and twisted flux in non-negligible, and possibly evolving, proportions (Figure 3(e)). Hybrids can arise in FCs by magnetic reconnection in the center of an SMA (Section 22 or between an SMA and its overlying potential loops or by the diversion of some of the current-carrying flux to localized polarities along the PIL (Section 3.1).

All three magnetic field configurations above are current-carrying structures loaded with free magnetic energy and magnetic helicity. Hence, they could all lead, under specific conditions, to eruptions. In the rest of the paper, we discuss these conditions, caveats and observational and modelling challenges. 


\section{Filament Channel Formation}

Filament channels are the cornerstones for understanding solar eruptive activity. FCs are sheared, and hence are reservoirs of magnetic free energy, which is required to fuel eruptive phenomena. CMEs occur only above PILs that are traced by an FC (all CME theories and models require the presence of an FC above a PIL). Hence, understanding FC formation equates to understanding the pre-eruptive configuration in the corona. FC properties are reviewed in several papers (e.g., Martin, 1998, Mackay, 2015).

The literature abounds with modeling efforts to explain the formation of the magnetic configuration of a filament channel (for a comprehensive review, see Mackay et al, 2010). The models can be differentiated between those employing surface effects (differential rotation, meridional flows, shear and converging flows, helicity condensation), and those employing sub-surface effects (the emergence of MFRs). In particular, FC could be formed by the following three mechanisms: (a) flux emergence, (b) flux cancellation, and (c) helicity condensation. Note, that frequently these mechanisms do not operate in isolation. Sections 2.1, 2.2 and 2.3 discuss these mechanisms and the corresponding observations in detail.

\subsection{Flux Emergence}

\subsubsection{Introduction}

A number of observational studies have shown that the formation of FCs is related, implicitly or explicitly, to the process of magnetic flux emergence from the solar interior into the solar atmosphere. There are studies suggesting that during flux emergence, a (twisted) flux tube, which rises from the solar interior, can emerge as a whole above the photosphere (e.g. Lites, 2005, Okamoto et al, 2008, Lites et al, 2010, Xu et al, 2012 Kuckein et al, 2012). This bodily emergence may result in a pre-eruptive MFR configuration. Other studies have reported that pre-eruptive structures can form along the strong PILs of ARs during flux emergence. For instance, the gradual build-up of free energy injected by photospheric motions can lead to shearing of the emerged magnetic field, forming an SMA. Numerical simulations of magnetic flux emergence indicate that such an SMA may eventually evolve into an MFR, which could erupt towards the outer solar atmosphere (e.g. Manchester et al, 2004, Archontis and Török, 2008, Archontis et al, 2009, Fan, 2009b Archontis and Hood, 2012 |Moreno-Insertis and Galsgaard, 2013; Leake et al, 2013, 2014; Syntelis et al, 2017, 2019b). Moreover, studies have reported the formation of FCs at the periphery of ARs or between neighbouring ARs (e.g. Gaizauskas et al, 1997; Wang and Muglach, 2007).

Here, we should highlight that the scope of this review is not an extended presentation of the various aspects of magnetic flux emergence, as a key process towards understanding the nature of solar magnetic activity. There exist several comprehensive reviews on this subject (e.g. Moreno-Insertis, 2007; Archontis, 2008, Fan, 2009a , Nordlund et al, 2009, Archontis, 2012; Hood et al, 2012, Stein, 2012, Cheung and Isobe, 2014; Toriumi, 2014, Archontis and Syntelis, 2019, Leenaarts, 2020). Thus, in the following sections, we mainly focus on the pre-eruptive struc- 
tures that form as a result of flux emergence, from a modelling (section 2.1.2) and an observational perspective (section 2.1.3).

\subsubsection{Flux Emergence Modeling}

Numerical experiments of magnetic flux emergence can be classified in two very broad categories, based on the nature of the background atmosphere that is included in the simulations. In the first category, the numerical domain contains the upper part of the solar atmosphere (e.g., the solar corona), and the magnetic flux is injected through the lower boundary of the domain, typically in the form of an MFR (e.g. Fan and Gibson, 2004). Such boundary-driven models are useful to study the stability and/or the eruptive behavior of coronal structures, but they do not capture the emergence process itself. In the second category, the magnetic field emerges from the solar interior and expands into a highly stratified atmosphere above the photosphere, driving the atmospheric dynamics self-consistently (e.g. Manchester, 2001, Fan, 2001, Archontis et al, 2004). A typical (idealized) numerical setup for the experiments in the second category consists of a convectively stable solar interior, a photospheric/chromospheric region of constant temperature and decreasing density, a region where the temperature increases rapidly with height, mimicking the temperature gradient of the transition region, and an isothermal corona. In this review, we will mainly focus on the models of the second category, as they are more suitable for studying the formation and evolution of pre-eruptive magnetic structures, at various atmospheric heights.

The most common initial condition for the sub-photospheric magnetic field is a twisted flux tube placed in the upper part of the solar interior, adopting the configuration of a straight horizontal tube or of a torus-shaped tube. Then, the emergence is initiated by imposing a density deficit along the tube, which makes part of the tube magnetically buoyant (e.g. Fan, 2001), or by imposing a velocity perturbation (e.g. Magara and Longcope, 2001, 2003) along a segment of the tube, which leads to the development of a rising loop with an $\Omega$-like shape (e.g. Archontis et al, 2004, Manchester et al, 2004). Toroidal flux tubes are typically used to mimic the top part of a sub-photospheric $\Omega$-shaped emerging loop (e.g. Hood et al, 2009, Cheung et al, 2010).

A considerable number of flux-emergence simulations have been using a subphotospheric horizontal magnetic flux sheet as initial condition. The interplay between the effect of convective motions on the magnetic field and the effect of the distorted field on the motion leads to the development of a series of small-scale interconnected $\Omega$-shaped loops (a 'sea-serpent' configuration Pariat et al, 2004)) that may eventually emerge through the photosphere (e.g. Isobe et al, 2007; Archontis and Hood, 2009, Toriumi and Yokoyama, 2010, Stein et al, 2011, Stein and Nordlund, 2012). These simulations have been used successfully to study small-scale dynamic phenomena such as Ellerman bombs and UV bursts (e.g., Danilovic et al, 2017, Hansteen et al, 2017, 2019) and the formation of complex bipolar regions and pores (e.g. Stein and Nordlund, 2012). However, these numerical experiments have not (yet) been able to produce large-scale pre-eruptive configurations or eruptions.

We note that a significant number of flux-emergence simulations incorporated additional physics, such as convective motions, radiative heating and cooling, heat conduction, ambipolar diffusion, ion-neutral interactions, and non-equilibrium ionization (e.g., Leake and Arber, 2006; Stein and Nordlund, 2006; Cameron et al, 
2007, Martínez-Sykora et al, 2008, Isobe et al, 2008, Cheung et al, 2010; Fang et al, 2010; Rempel and Cheung, 2014; Chen et al, 2017; Hansteen et al, 2017; MorenoInsertis et al, 2018, Nóbrega-Siverio et al, 2018, Cheung et al, 2019, Toriumi and Hotta, 2019). These simulations are necessary for studying the thermodynamical aspects of phenomena related to flux emergence and the atmospheric response to the dynamic emergence of solar magnetic fields. However, the vast majority of these experiments have not yet included the solar corona to an extent that is required to model pre-eruptive configurations.

In the following, we will review the formation and evolution of the most common pre-eruptive configurations that are found in flux-emergence simulations.

\section{Partial and bodily emergence of MFRs}

Numerical simulations have shown that the rise of an initially horizontal subphotospheric flux tube is usually not followed by the bodily emergence of the tube into the higher atmosphere (e.g., Fan, 2001, Magara and Longcope, 2003, Manchester et al, 2004). When the top part of a flux tube starts to emerge into the atmosphere, it expands rapidly due to the pressure difference between itself and the solar atmosphere. During this expansion, a region with low density and pressure is formed at the centre of the emerging region, at photospheric heights. Plasma then drains along the field lines, moving from the top part of the flux tube towards this low-pressure region (e.g. Manchester et al, 2004) and, eventually, it accumulates in the dips of the twisted field lines. The dips become heavier and, thus, the axis of the tube cannot emerge fully into the corona. Rather, it reaches only a few pressure scale heights above the photosphere. Full (bodily) emergence can occur only if the tube is buoyant enough to reach the top of the low pressure region, before the drained plasma accumulates at its dips.

Magara and Longcope (2003) demonstrated how the curvature of different field lines within a horizontal flux tube, which emerged from just below the photosphere $(-2.1 \mathrm{Mm})$, affected the draining of plasma. Murray et al (2006) performed a parametric study in a similar setup, and varied the initial twist (affecting plasma draining) and the initial magnetic field strength (affecting the buoyancy) of the sub-photospheric flux tube. More draining and more buoyancy assisted the axis of the tube to move higher inside the photosphere. MacTaggart and Hood (2009) studied a case where the middle part of the tube was emerging and the flanks were submerging. This led to more efficient draining along the flanks of the tube but it didn't trigger bodily emergence.

In all the above-mentioned studies, the initial location of the emerging flux tube was near the photosphere. Syntelis et al (2019a) imposed horizontal flux tubes deeper in the solar interior $(-18 \mathrm{Mm})$, to allow the tubes to develop a more strongly curved shape as they emerge towards the photosphere. They performed a large parametric study, varying the magnetic field strength, radius, twist, and length of the buoyant segment of the tube (a proxy for the curvature). They found that the axis of the flux tube always remained below the photosphere. In addition, they showed that it is non-trivial to predict the combined effects of these parameters during the emergence of the field. For instance, a non-intuitive result was that high-strength (weak-strength) flux tubes may fail (succeed) to emerge into the atmosphere, depending on their geometrical properties.

On the other hand, Hood et al (2009) studied the emergence of a weakly twisted, toroidal flux tube. They found that the geometrical shape of the tube 
(higher curvature), and the smaller number of dips along the twisted field lines, triggered sufficient plasma draining and buoyancy, which helped the tube to emerge bodily into the corona, given that the initial field strength in the tube was chosen sufficiently strong. MacTaggart and Hood (2009) systematically varied the field strength and found cases where the axis of the tube (i) could not break through the photosphere, (ii) emerged but stayed within the photospheric layer, and (iii) reached coronal heights and continued rising (i.e., emerged bodily). The bodily emerged MFRs in these simulations appear to be weakly twisted (about one turn or less), but the dependence of the MFR twist on the initial field strength or twist of the sub-photospheric flux tube has not yet been studied systematically. Both Hood et al (2009) and MacTaggart and Hood (2009) reported that a second MFR formed via magnetic reconnection (see below for a detailed description of this process) underneath the bodily emerged MFR. If, on the other hand, the original axis did not emerge into the corona, the second MFR was seen to form above it (see Figure 4).

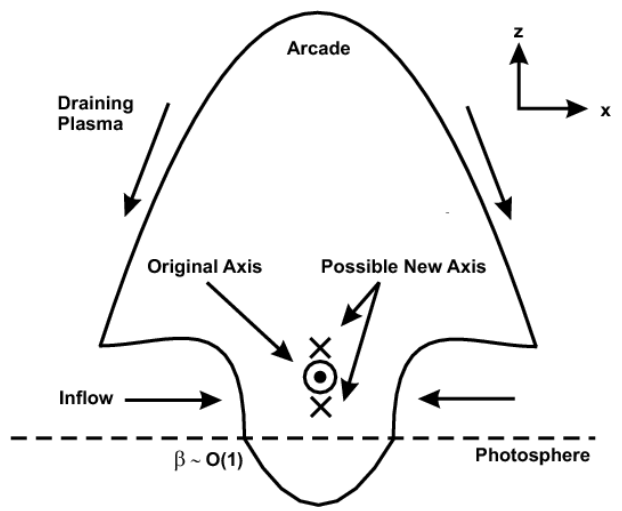

Fig. 4 Creation of converging flows during the partial emergence of a sub-surface MFR and subsequent reorganization of the coronal field, which produces a new MFR. Reproduced with permission from A\&A, CESO. From MacTaggart and Hood (2009).

The numerical experiments summarized in this section suggest that whether a sub-photospheric twisted flux tube emerges bodily, partially, or not at all, depends mainly on the properties of the rising magnetic field and its geometric configuration. It appears that bodily emergence requires rather specific conditions, namely a toroidal geometry and a relatively large field strength. We discuss observational evidence for bodily emergence in Section 2.1.3. Next, we focus on the formation of MFRs via magnetic reconnection, as seen in flux-emergence simulations.

\section{MFR formation via reconnection}

The emergence of a single sub-photospheric flux tube typically leads to the formation of a bipolar region, whose polarities separate over time. Photospheric motions commonly found in the vicinity of the region's PIL include: (i) shearing along the PIL, resulting from the Lorentz force developed at the photosphere due to the expansion of the emerging field (e.g., Fan, 2001; Manchester, 2001), (ii) rotation of the polarities driven by the propagation of a torsional Alfvén wave 
into the atmosphere (e.g., Longcope and Klapper, 1997, Fan, 2009b; Leake et al, 2013 Sturrock et al, 2015, Sturrock and Hood, 2016), and (iii) downflow of plasma towards the low-pressure region above the PIL as discussed above (e.g., Manchester et al, 2004). The combination of these motions induces converging flows towards the PIL (e.g., Archontis and Hood, 2010, Syntelis et al, 2017), which ultimately lead to the formation of an MFR.

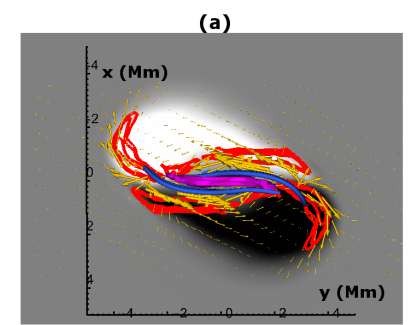

(c)

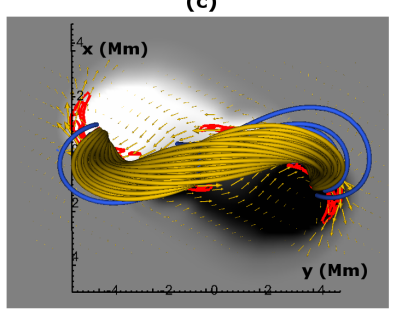

(e)

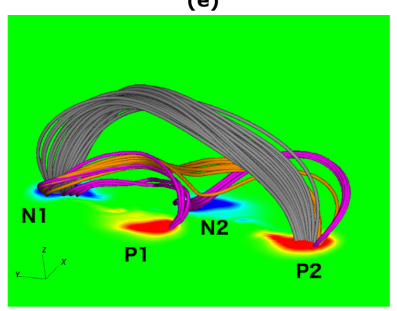

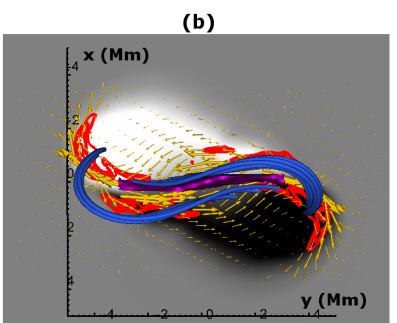

(d)

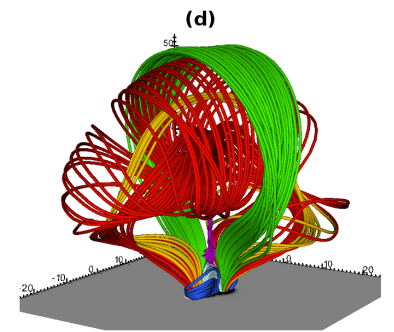

(f)

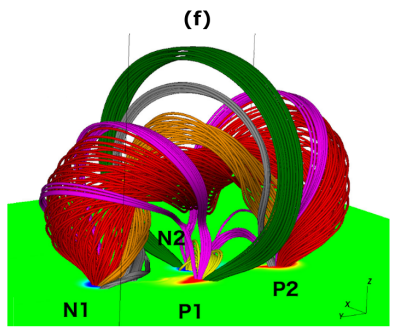

Fig. 5 MFR formation by reconnection in bipolar and quadropolar regions. (a, b) Top view on field lines that become increasingly sheared and adopt a double-J-like shape (blue lines). The horizontal slice shows the distribution of $B_{z}$ (black and white) at the photosphere. Overplotted are yellow arrows showing the photospheric velocity field scaled by magnitude, red contours showing the photospheric vorticity and a purple isosurface showing $|\mathbf{J}| /|\mathbf{B}|=0.3$ (c) Field lines of the sigmoidal MFR formed by reconnected J-like field lines (orange). (d) The field line topology during the eruption of the MFR. The red lines show tether-cut field lines due to reconnection, the green lines show the remaining envelope field which has not yet reconnected and the cyan lines show the post-reconnection arcade. The purple isosurface shows the flare CS. (e) The topology of the field lines in a quadrupolar region. Pink lines show the field lines of the two interacting magnetic lobes. Orange lines have been traced from the close vicinity of the core of the MFR. Field lines in grey color show the "envelope" field resulting from reconnection between the two magnetic lobes. (f) The field line topology during the rise of the MFR, which is eventually confined by the envelope field. The red lines are field lines, which have been reconnected during the interaction of the two magnetic lobes and the formation and rise of the MFR. The green and grey field lines lines belong to the envelope field above the MFR. The low-lying yellow field lines are reconnected field lines, which form a system of post-reconnection arcade loops that connect the inner polarities (P1, N2).(from Syntelis et al (2017) and Syntelis et al (2019c) ). CAAS. Reproduced with permission. 
This is illustrated in Figure 5. The described motions inject free magnetic energy into the system and lead to the gradual formation of an SMA above the PIL. Eventually, a strong, vertical current layer is formed within the SMA (Figure 5 a), the sheared field lines adopt a double-J shape (Figure $5 \mathrm{~b}$ ), and the current layer's strength increases. Field lines that belong to the SMA reconnect above the PIL and form a (new) MFR with a sigmoidal shape (Figure 5k) at low atmospheric heights. Spectroscopic observations by CDS,EIS and IRIS, taken at the footprints of forming MFRs, showed significant line-shifts and line-broadenings, implying the occurrence of reconnection in the low atmosphere (Foley et al, 2001, Harra et al, 2013. Cheng et al, 2015).

The newly formed MFRs are typically weakly twisted (approximately one turn, although the exact number of turns has not been measured in most studies) and can become eruptive (Figure 5d). Overall, a large number of numerical experiments (e.g. Manchester et al, 2004 Archontis and Török, 2008, Archontis et al, 2009, Fan, 2009b Archontis and Hood, 2012, Leake et al, 2013, Moreno-Insertis and Galsgaard, 2013 Archontis et al, 2014, Fang et al, 2014, Leake et al, 2014; Lee et al, 2015: Syntelis et al, 2017, Toriumi and Takasao, 2017, Syntelis et al, 2019b c) have shown that post-emergence MFRs can form through reconnection of sheared magnetic field lines across a current layer above the PIL of an emerging AR, in the same manner as suggested for the formation of large-scale (quiescent) FCs suggested by van Ballegooijen and Martens (1989); see also Section 2.2

Flux-emergence models have been used to study also the dynamic evolution at the PILs between colliding/interacting bipoles in quadrupolar ARs (e.g. Fang and Fan, 2015, Takasao et al, 2015, Toriumi and Takasao, 2017; Cheung et al, 2019, Syntelis et al, 2019c). We note that the nature of shear and its role for the formation and eruption of MFRs has been investigated also in observational studies, for cases of two colliding emerging bipoles (Chintzoglou et al, 2019). Here we focus on the formation of MFRs in quadrupolar ARs in flux-emergence simulations.

The most common initial set up of the numerical simulations on this subject include the emergence of either: (i) a single $\Omega$-loop flux tube with low twist (e.g. Murray et al, 2006, Archontis et al, 2013, Syntelis et al, 2015); (ii) a single flux tube emerging at two different locations along its length ("double- $\Omega$ " loop), so that two nearby bipoles appear at the photosphere (e.g. Fang and Fan, 2015, Lee et al, 2015, Toriumi and Takasao, 2017); (iii) two different $\Omega$-loop flux tubes emerging nearby (e.g. Toriumi et al, 2014 Toriumi and Takasao, 2017); (iv) a single kink unstable flux tube (such flux tubes, depending on the twist, can form from bipolar to more complex multipolar configurations) (e.g. Takasao et al, 2015, Toriumi and Takasao, 2017, Knizhnik et al, 2018). In the above cases, a PIL is formed between the inner-most polarities of the quadrupole, above which a strong current layer can build up and a MFR can be formed. Such MFRs can potentially erupt.

Syntelis et al (2019c) reported on a model of recurrent confined eruptions in a quadrupolar region. Initially, prior to the formation of the MFR, the two magnetic lobes above the two emerged bipoles were not interacting. As the bipoles emerged and moved, the inner polarities of the quadrupole approached each other, and a current sheet (CS) formed above the PIL between them, extending between the two magnetic lobes. The strength and size of this CS progressively increased over time. Reconnection between the two magnetic lobes through that CS formed a magnetic "envelope" above the quadrupolar region and a weakly-twisted (approximately one turn) post-emergence low-lying MFR (Figure 5p). This pre-eruptive MFR did 
not result from the internal reconnection of a SMA. Rather, the MFR formed directly. The eruption of the MFR was triggered by reconnection internally within the quadrupolar region, occurring both below and above the MFR. During the confined eruption, the weakly twisted low-lying MFR became a larger and more twisted confined coronal MFR, similar to confined-flare-to-flux-rope observations (e.g. Patsourakos et al, 2013). The eruptivity of the latter MFR was not studied.

Post-emergence MFRs can form in a recurrent manner as long as the photospheric motions are present and magnetic energy is injected into the system (e.g. Moreno-Insertis and Galsgaard, 2013; Archontis et al, 2014; Syntelis et al, 2017, $2019 \mathrm{~b}$ c). When the driving motions are no longer able to build enough free energy, the recurrent formation of post-emergence MFRs ceases. It is important to note here that in flux-emergence models, the formation of MFRs and subsequent eruptions do not occur only during the flux-emergence phase (i.e., while the photospheric flux still increases). They can also occur when the photospheric flux has saturated, but the photospheric motions are still present (e.g, the vertical dashed lines in Figure 20 in the Appendix).

As we have mentioned previously in this section, the pre-eruptive MFRs are typically weakly twisted. However, we should highlight that the number of turns of the field lines resulting from the reconnection between two flux systems depends on the specifics of the reconnection region. For instance, Wright (2019) showed that: a) the relative orientation of the footpoints of the two pre-reconnection flux systems and b) their magnetic helicity content (for a discussion on magnetic helicity see Section 2.3), are crucial to determine the resulting twist after they reconnect to each other. These two factors affect how self-helicity is partitioned between the resulting post-reconnection systems. Wright (2019) discussed cases where the twist of the resulting two flux systems can increase, decrease or remain the same after their interaction. Priest and Longcope (2020) further studied how self-helicity is partitioned between reconnecting systems, by examining twist during the reconnection of flux sheets, sheaths and tubes, and discussed how the twist of the erupting MFR increases during the eruption, leaving behind an untwisted arcade. Multiple reconnection events between different flux systems can furthermore increase/decrease the twist of a pre-eruptive MFR. This can occur when the post-reconnected field lines reconnect again with field from the same pre-reconnected systems, or when they reconnect with other flux systems. Similarly, during an eruption, the MFR twist typically increases due to multiple reconnection events in the flare CS below the core of the erupting MFR (e.g., red lines in Fig. 5 d and Gibson and Fan (2006b); Syntelis et al (2017); Inoue et al (2018); Syntelis et al (2019b)).

MFRs could also form at the periphery (or in between) ARs as was recently suggested by Török et al (2018b). They modeled the emergence of an MFR close to a pre-existing bipolar AR. The orientation of the MFR was chosen such that a quadrupolar configuration with a current layer between the pre-existing and newly emerged flux systems resulted, similar to the configurations just described. A pair of so-called "conjoined flux ropes" (CFRs; e.g., Wyper and Pontin, 2014b a t Titov et al, 2017; Fu et al, 2017) was created by the tearing instability in the current layer and advected to the lower atmosphere, leading to the formation of two MFRs of opposite axial-field direction (i.e., opposite helicity sign) that are located endto-end above the "external" PIL section between the new and pre-existing flux (Figure 6). Note that the CFRs form in addition to the main MFR that bodily emerges or forms above the "internal" PIL of the emerging region, as described 


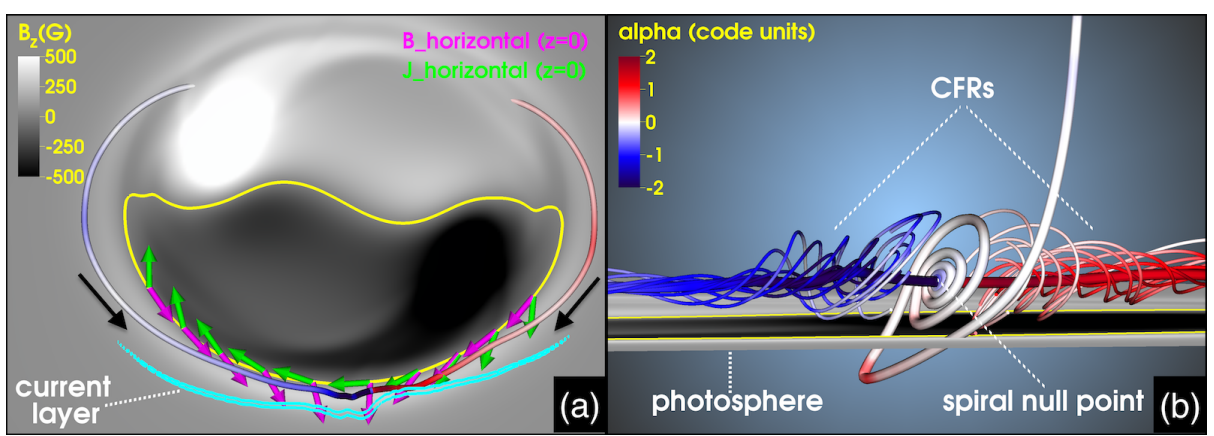

Fig. 6 MHD simulation of FC formation at the periphery of an emerging AR. (a) Top view on the AR, which emerges into a positive background field, close to a pre-existing AR (not shown). The yellow line marks the PIL, arrows show the horizontal magnetic field (magenta) and current density (green) along the external PIL section. The magnetic field switches sign along and above this section (indicated by black arrows), leading to the formation of CFRs (i.e., two adjacent MFRs of opposite helicity sign) via reconnection across the current layer (indicated in cyan). (b) Oblique view on the MFRs, colored by $\alpha=(\mathbf{j} \cdot \mathbf{B}) / B^{2}$. The location of the spiral null point between the two MFRs is illustrated by a thick field line.

above. Note also that the presence of a pre-existing bipole is not required for this mechanism to work, as the current layer forms between the inner polarities. That is, the mechanism can work also when an AR emerges within, or close to, a single-polarity region, i.e., a coronal hole.

\subsubsection{Flux emergence observations}

We start with observations of FC formation within the cores of emerging flux regions interpreted in favor of bodily emerging MFRs. Lites et al (1995) studied the emergence and evolution of a small $\delta$-spot AR, where a low-lying filament was seen in $\mathrm{H} \alpha$ above the PIL. They found that a "magnetically closed structure" remained in the corona even after the disappearance of the $\delta$-spot AR. They attributed this to an MFR in equilibrium with the ambient coronal field, after it had emerged through the photosphere. Later works revisited the emerging MFR scenario by examining the vector magnetic field in locations where filaments form in plages, at the photosphere (Lites, 2005, Okamoto et al, 2008; Lites et al, 2010) and by also including chromospheric vector magnetograms (Kuckein et al, 2012| |Xu et al, 2012). The photospheric vector field in these works is found to be of a "concaveup" geometry suggesting a magnetic structure dipping at the PIL, and also that of an "inverse" horizontal field (with respect to that of a potential field, which is called "normal") crossing the PIL. To our knowledge, these works are the only references suggesting full (i.e., bodily) MFR emergence, making it a rather scarce observation.

However, several important observational limitations (e.g., incomplete spectral and temporal coverage or confusion from pre-existing structures and the lack of multi-height vector magnetic field measurements), do not allow to unambiguously interpret observations of flux emergence. Therefore, their interpretation in terms of bodily emerging MFRs could not be unique. 
For example, several analyses take place while a filament already exists in these locations (Okamoto et al, 2008; Kuckein et al, 2012), casting doubt that these are studies of true FC formation. In addition, MHD modeling of dynamic emergence of a twisted cylinder into an overlying arcade by Vargas Domínguez et al (2012) showed the same photospheric signatures as observed by Okamoto et al (2008). However, this was not a case of a bodily-emerging flux rope into the corona, since the axis of the emerging cylinder reached only one photospheric scale-height above the photosphere. This result reveals that the concave upward geometry is not an exclusive indication of a bodily emerging MFR. The $\mathrm{Xu}$ et al (2012) study was based on the analysis of a single chromospheric magnetogram (in HeI $10830 \AA$ ) and comparison to $\mathrm{H} \alpha$. They found that the chromospheric magnetic field exhibited the normal configuration while the photospheric magnetic field was concave upwards and these results were interpreted in terms of bodily emergence of a flux rope that is producing a filament. The authors acknowledge that their interpretation is not unambiguous given that the observed differences in HeI 10830 $\AA$ and $\mathrm{H} \alpha$ may be interpreted in terms of optical-depth effects. The same issue affects the Kuckein et al (2012) results since they used the same lines. Mackay et al (2010) analyzed the same sequence as Okamoto et al (2008) but interpret the signatures in the Ca II H line being due to a rising SMA rather than an MFR. Lites et al (2010) discuss the possibility of surface flows creating their observed short-lived filament channel via cancellation, but they reject it based on the lack of systematic photospheric flows that could produce significant shear and convergence along the channel. However, their flow field was determined from the photospheric granulation instead of tracking magnetic elements in magnetograms. Therefore, MFR formation by flux cancellation (see next section) remains a candidate.

We now pass into a discussion of observations of FC formation in the periphery of ARs, rather in their cores, including quiet Sun (QS) filaments. AR periphery is indeed where most filament channels form. Observations, (e.g., Gaizauskas et al, 1997, Wang and Muglach, 2007), suggest that such filament channels form during mainly interactions of emerging bipoles with existing ones. The bipoles converge and flux cancellation and reconnection take place. These scant observations, along with the considerations of the solar-cycle characteristics of filament channels within and outside ARs, with only the latter showing solar-cycle dependence, led Mackay et al (2010) to conclude that emerging flux ropes are not relevant for the formation of filament channels outside ARs, and "surface" phenomena, i.e., converge and cancellation are more pertinent. We finally note here, that the observational inferences of bodily emerging flux ropes within AR cores discussed in the previous paragraphs, may not be attainable for the case of filament formation outside ARs, given the weaker (horizontal) magnetic fields in these areas.

Flux emergence in multipolar ARs can eventually lead to the formation of MFRs (Chintzoglou et al, 2019). These authors reported observations of multiple bipoles, emerging either simultaneously or sequentially, in two emerging ARs. The collision between oppositely signed nonconjugated polarities (i.e., polarities not belonging to the same bipole) of the different emerging bipoles within the same AR gave rise to shear and flux cancellation, hence this process was called collisional shearing. Photospheric flux cancellation and reconnection above the photosphere in the PIL(s) undergoing collisional shearing progressively converted SMAs into pre-eruptive MFRs and produced intense flare clusters for the duration of collision. In the same study, a data-driven evolutionary magneto-frictional model (e.g., 
Fisher et al, 2015) was applied to time-series of photospheric vector magnetic field and Doppler measurements of the two analyzed ARs, and it was able to capture and reproduce the various stages of collisional shearing, including the progressive conversion of SMAs to pre-eruptive MFRs. In addition, using Doppler observations it was determined that the active part of the AR PIL showed downflows, further supporting that the pre-eruptive MFRs did not emerge bodily and were produced by strong cancellation.

\subsubsection{Outstanding Issues}

We conclude this section with a list of pending issues with regards to flux emergence simulations and observations:

- improve the physical realism of flux emergence simulations;

- lack of systematic studies of full and partial flux-tube emergence ;

- lack of systematic calculations of twist in flux emergence simulations;

- properties and eruptivity of bodily emerging MFRs has not been studied yet;

- lack of extensive surveys looking for bodily emerging MFRs ;

- lack of extensive surveys of FC formation at AR peripheries.

A detailed roadmap towards addressing these issues is given in Section 5 .

\subsection{Flux Cancellation}

\subsubsection{Introduction}

Magnetic flux cancellation, whereby small-scale opposite magnetic polarities converge, collide and then subsequently disappear (Martin et al, 1985) is a process ubiquitous all over the solar photosphere (Livi et al, 1985). Flux cancellation takes place along the PIL (Babcock and Babcock, 1955) that separates negative and positive photospheric polarities. In particular, cancellation can occur along the internal PIL of an AR, at an external PIL formed at the AR periphery, or in the QS. According to the classical picture of magnetic flux cancellation described in van Ballegooijen and Martens (1989) it invariably leads to the formation of an MFR. A different model for magnetic cancellation is discussed in Section 2.3, and when combined with helicity condensation, this type of cancellation leads to an SMA.

\subsubsection{Flux cancellation modeling}

Flux cancellation due to magnetic reconnection at the surface is typically modeled by modifying the lower boundary of a simulation domain that represents the photosphere. For example, in the work of Amari et al (1999); Linker et al (2001); Lionello et al (2001), a potential-field extrapolation of either an idealized or observed AR magnetic field is used as the initial condition. This field is then energized by surface flows, such as shearing or twisting, followed by diffusion of the normal magnetic field on the surface, which is achieved by imposing an appropriate tangential electric field. The ensuing evolution produces a coronal MFR with dipped field lines that could support filament material. In the model of DeVore and Antiochos (2000), no specific surface flux cancellation is specified. Instead, the expansion due to footpoint motions which increase the pressure of the sheared 
magnetic field in the corona leads to magnetic reconnection, similar to the theoretical mechanism of van Ballegooijen and Martens (1989). Finally, in the models of Yeates et al (2008) and van Ballegooijen et al (2000), diffusion of the magnetic field at the coronal base (with the simultaneous absence of magnetic diffusion in the corona) concentrates magnetic field just above PILs, which becomes the axial field of the MFR formed by the subsequent reconnection.

The complex physical mechanisms associated with flux emergence are reviewed in Section 2.1. During the partial emergence of magnetic flux (where mass-laden portions of magnetic flux tubes remain rooted at the surface), the Lorentz force associated with expanding field lines that are able to drain material produces shear flows at and above the PIL (Manchester et al, 2004). In addition, because of the rapid horizontal expansion of the magnetic field at the $\beta=1$ surface (see Figure 4), convergent flows can also be created by the self-consistent evolution of the magnetic field in the low atmosphere, leading to magnetic reconnection at those locations (MacTaggart and Hood, 2009). Furthermore, the emergence of concaveup field lines (U-loops) can occur when the magnetic field becomes significantly distorted during its emergence in the turbulent convection zone (Magara, 2011). Therefore, flux emergence can create the observational signatures of shearing and converging flows, and of flux cancellation.

Recently, the ability to include into the simulations the physical mechanisms of both flux emergence and flux cancellation due to both magnetic-field evolution and magneto-convection has been developed. By mimicking the radiative losses at the surface and including a thermal injection of energy at the base of the simulation domain, MHD simulations spanning a shallow convection zone to lower corona can now include reasonably realistic convective motions. Fang et al (2012) address the emergence of MFRs modified by the turbulent convection zone. Within the emerging structure, converging motions at the PIL, driven by a combination of magnetic-field evolution and granular motion, cause flux cancellation at the photosphere, which, along with tether-cutting reconnection in the corona, continues to build up sheared field lines in the corona. This type of study, performed on realistic solar AR timescales may help to understand what causes the flux cancellation observed prior to internal filament eruptions.

Convective motions at the surface may also aid in the formation of filament channels both within and external to an AR, via the recent theoretical model of helicity condensation, discussed in Section 2.3 .

\subsubsection{Flux cancellation observations}

The convergence of opposite magnetic polarities towards PILs that leads to flux cancellation is driven by the dispersion of decaying AR magnetic fields, through convection and emergence of AR bipoles into pre-existing magnetic field, and also by the collision of bipoles during the emergence of multipolar ARs. The process of flux cancellation is often observed leading up to the formation of filament channels, filaments and CMEs (Martin et al, 1985, 2012, Martin, 1998, Gaizauskas et al, 2001, Gaizauskas, 2002, Wang and Muglach, 2007, Mackay et al, 2008, 2014, Chintzoglou et al, 2019), suggesting that flux cancellation plays an important role in the construction of pre-eruptive magnetic-field configurations. Small-scale brightenings and jets have been observed in the corona in connection with cancellation sites (e.g., Wang and Muglach, 2013). However, such brightenings are 
most commonly observed in $\mathrm{H} \alpha$ and $\mathrm{He}$ II, with fainter or no signatures in coronal emissions.

There are currently three proposed scenarios that describe the physical processes that lead to flux cancellation (Zwaan, 1987): U-loop emergence (van DrielGesztelyi et al, 2000, Bernasconi et al, 2002), $\Omega$-loop submergence (Harvey et al, 1999, Chae et al, 2004 Yang et al, 2009, Takizawa et al, 2012), and magnetic reconnection taking place low in the solar atmosphere followed by $\Omega$-loop submergence (van Ballegooijen and Martens, 1989). Current observations cannot easily distinguish between these possibilities.

In the case that flux cancellation is associated with magnetic reconnection, as in the van Ballegooijen and Martens (1989) model, then sheared magnetic fields reconnect in the PIL leading to the formation of twisted field lines pertinent to a MFR. More specifically, the sheared field evolves from an SMA to two sets of loops that form a "double-J" shape with a low-emission channel between them. The low emission channel is expected to contain cooler plasma (about 1MK), but this has not yet been detected. From this configuration, the inner end points of the two J's merge and a continuous S-shape forms, but this may also be a projection effect. This continuous S-shape corresponds to a SXR or extreme ultraviolet (EUV) sigmoid. Green and Kliem (2014) show this evolutionary scenario for four ARs, with the transition from SMA to double-J to continuous S-shape typically taking a couple of days. The continuous S structure is highly supportive of the presence of an MFR with helical field lines with around one turn, where heated plasma is confined. However, the MFR may also be present at the time of the two J's. The double-Js then represent remnant SMA field that is wrapping around the forming MFR but which has not yet undergone reconnection and been built into the MFR.

During flux cancellation an amount of flux equal to the amount removed from the photosphere is available to be built into an MFR. Therefore, flux cancellation observations present a way to investigate how much magnetic flux has been built into an MFR before its eruption and we hereby discuss such estimates. Previous observational studies of flux cancellation monitoring the magnetic flux evolution in the photosphere, suggest that around $30-50 \%$ of the AR flux cancels on average 2-4 days prior to the occurrence of a CME (Green et al, 2011; Baker et al, 2012, Yardley et al, 2016, 2018a). However, these values represent an upper limit as the amount of flux that is built into an MFR is dependent on the AR properties, such as the shear of the magnetic field and the length of the PIL along which flux cancellation is taking place (Green et al, 2011).

In addition, static or time-dependent (non-linear force-free field; NLFFF) models provide an alternative method to probe how much flux is contained in an MFR before its eruption. The models include the flux rope insertion method (van Ballegooijen, 2004) to produce a NLFFF extrapolation or the NLFFF evolutionary model of Mackay et al (2011), to model pre-eruptive configuration of the magnetic field. Using these models it is possible to investigate the ratio of axial and poloidal (i.e., at planes perpendicular to the axis ) flux in the MFR to that of the overlying field for stable and unstable MFRs. Previously, the limit for the axial flux in an MFR that can be held in stable force-free equilibrium by the overlying field of the $\mathrm{AR}$ was found to be up to 10-14\% (Bobra et al, 2008, Su et al, 2009, Savcheva and van Ballegooijen, 2009). On the other hand, more recent studies that combine both observations and models have suggested that $20-50 \%$ of the AR flux could be contained in a stable MFR (Savcheva et al, 2012, Gibb et al, 2014). There may be 
also poloidal magnetic flux added to the MFR via reconnection in the transition region or corona, but lack of routine magnetic field observations at these layers prevents any direct assessment of it.

For sigmoids that form along the internal PIL of decaying ARs, the coronal field appears to follow a systematic evolution when viewed in SXR observations. These regions match closely the mechanism of van Ballegooijen and Martens (1989) in that they exhibit flux cancellation along the PIL and an increasingly sheared field in the corona. A recent study by (Savcheva et al, 2014) has shown that the eruptive activity of 72 sigmoidal ARs is more strongly correlated with flux cancellation than with emergence. The study found that $57 \%$ of the sigmoids were associated with flux cancellation compared with only $35 \%$ occurring as a result of flux emergence. The rest of the regions showed a fairly constant magnetic flux evolution.

A secondary effect of flux cancellation is that it reduces the magnetic flux that contributes to the overlying, stabilizing field of the MFR. If enough flux is cancelled from the overlying field and incorporated into an MFR, a force imbalance occurs, which leads to a catastrophic loss of equilibrium and a CME (e.g., Lin and Forbes, 2000, Bobra et al, 2008). Or, if the overlying field of the AR decays rapidly enough with height, the flux rope can become torus-unstable (Kliem and Török, 2006: Török and Kliem, 2007, Démoulin and Aulanier, 2010, Kliem et al, 2014a). Previous observational studies of flux cancellation have found a ratio of flux contained in the MFR compared to the overlying arcade of 1:1.5 (Green et al, 2011) and 1:0.9 (Yardley et al, 2016). Yardley et al (2018a) recently conducted a comprehensive study of the CME productivity in a sample of 20 bipolar ARs in order to probe the role of flux cancellation as a CME trigger. The magnetic flux evolution was analyzed during the full AR evolution spanning from emergence to decay. This study found that the ratio of flux cancelled available to be built into an MFR before eruption compared to the remaining, overlying field was found in the range 1:0.03 to 1:1.57 for ARs that produced low-altitude CMEs originating from the internal PIL. The small ratios imply that the assumption that the amount of flux cancelled is equal to the amount of flux injected into the flux rope does not necessarily apply here. This needs further investigation. They suggest that a combination of the convergence of the polarities, magnetic shear and flux cancellation are required to build a pre-eruptive configuration and that a successful eruption depends upon the removal of a sufficient amount of the overlying field that stabilizes the configuration. The study also showed that the type of CME produced depends upon the evolutionary stage of the AR. CMEs originating above external PILs (between the periphery of the AR and the QS) occurred during the emergence phase of the AR, whereas, CMEs originating above an internal PIL of an AR occurred during the region's decay phase (see Figure 7).

Note that the majority of the observational studies of flux cancellation discussed above refer to bipolar and decaying ARs. Flux cancellation can also occur in multipolar and emerging ARs (Chintzoglou et al (2019) and discussion of Section 2.1.3). We note that measuring flux cancellation in periods of flux emergence is challenging (in comparison to measuring cancellation in decaying ARs) because the magnetic flux is increasing during the emergence phase. By tracking opposite polarity footpoints and the flux balance of multiple emerging bipoles within the same AR undergoing collisional shearing (see Section 2.1.3) it was found by Chintzoglou et al $(2019)$ that amounts of magnetic flux of up to $\approx 40 \%$ of the net magnetic flux of the smaller emerging bipole may be cancelled. The reported 
collisional shearing and magnetic cancellation preceded major eruptive activity in the analyzed ARs.

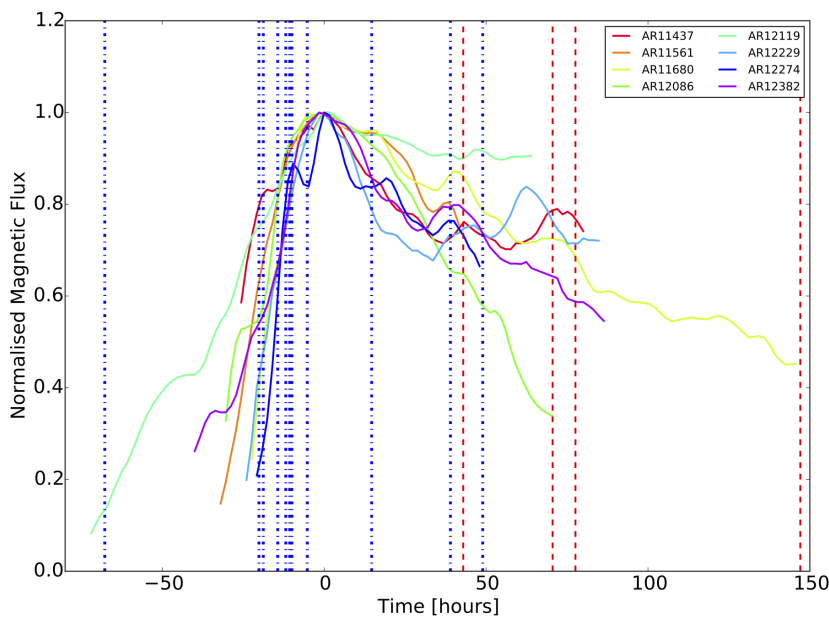

Fig. 7 The temporal evolution of normalized magnetic flux in CME-producing ARs studied by Yardley et al (2018a). The vertical lines indicate the timing of CME eruptions from PILs that are internal to the AR (red dashed lines) and PILs that are external to the AR (blue dot dashed lines). CMEs from external PILs tend to happen during the flux emergence phase $(\mathrm{t}$ $<0 \mathrm{hr}$ ) while CMEs from ARs' internal PILs happen well after the emergence phase ( $\mathrm{t}>0$ hr). (C)AAS. Reproduced with permission.

\subsubsection{Outstanding Issues}

We conclude this section with a list of pending issues with regards to flux cancellation modeling and observations:

- there are multiple physical processes that could be associated with flux cancellation and it is not clear which dominates (if any);

- the exact amount of flux that builds into the pre-eruptive magnetic configuration associated with flux cancellation is not fully constrained;

- the exact atmospheric height at which the magnetic reconnection that could be associated with flux cancellation occurs is still unknown;

-it is difficult to follow the full magnetic field evolution of ARs from emergence to decay, particularly for large or complex ARs, due to current observational limitations;

-flux cancellation during episodes of flux emergence needs to be taken into account.

A detailed roadmap towards addressing these issues is given in Section 5 . 


\subsection{Helicity Condensation}

\subsubsection{Introduction}

In recent years, a third mechanism has been proposed for the formation of filament channels, the so-called condensation of magnetic helicity at PILs (Antiochos, 2013). Magnetic helicity is the topological measure of twist, writhe, and the linkage of magnetic field lines (e.g., the review of Pevtsov et al, 2014). An attractive property of magnetic helicity is that it is conserved even under magnetic reconnection (e.g., Berger, 1984). Furthermore, for a turbulent system, helicity has been shown to undergo an inverse cascading toward larger spatial scales. The helicity condensation model implies that magnetic shear accumulates due to this helicity cascading in a given coronal flux system. The primary assumption is that a net helicity is injected into every coronal flux region by the continuous large and small-scale photospheric motions and associated flux emergence and submergence through the photosphere. It has been long observed that large-scale magnetic structures in the corona, such as sunspot whirls, exhibit a pronounced hemispheric helicity preference that was first postulated by Seehafer (1990) and assesed in photospheric magnetic field observations by Pevtsov et al (1995) (see Pevtsov et al 2014 for more recent references). This preference is especially strong (>90\%) for erupting filaments (Ouyang et al, 2017). A secondary requirement for the helicity-condensation model is that the coronal magnetic field is constantly undergoing turbulent-like reconnection, as in the nanoflare model (Parker, 1983, Klimchuk, 2006) for coronal heating. Given the observed constant small-scale convective flows and flux emergence/cancellation at the photosphere, it seems inevitable that the corona must be in a state of turbulent reconnection, irrespective of whether this process is important for coronal heating. Such resistive MHD turbulence is expected to produce an inverse cascade in the helicity due to helicity conservation even under non-ideal processes.

Inverse cascading is a well-known phenomenon in laboratory plasmas, and generally leads to the "self-organization" of a turbulent system (Biskamp, 1993). The helicity condensation model argues that for the corona self-organization manifests itself as the formation of sheared filament channels about PILs. Simulations by Knizhnik et al (2017) demonstrate the basic mechanism of the model. The left panel of Figure 8 shows the initial coronal system consisting of a potential magnetic field due to a bipolar flux distribution at the bottom plane (the photosphere), with a circular positive polarity region surrounded by a negative polarity. The blue circle in Figure 8 indicates the PIL. Three sets of field lines are shown: yellow lines that are open so that any stress by the photosphere simply propagates outward, black corresponding to typical coronal loops, and low-lying white lines very near the PIL. This initially zero helicity system was driven by small-scale free energy and helicity injection due to a simple photospheric flow pattern consisting of multiple rotations. The rotations filled the positive polarity region, thereby driving all the flux in the system. To reproduce the stochastic nature of photospheric convective flows, the rotations were randomly shifted about the bottom plane after every half-turn or so.

The right Panel of Figure 8 shows the end state of the system after approximately 90 rotations and demonstrates a striking example of self-organization. As expected, the yellow open field lines are relatively unchanged, but the black lines in the central portions of the flux system also show negligible shear or twist even 


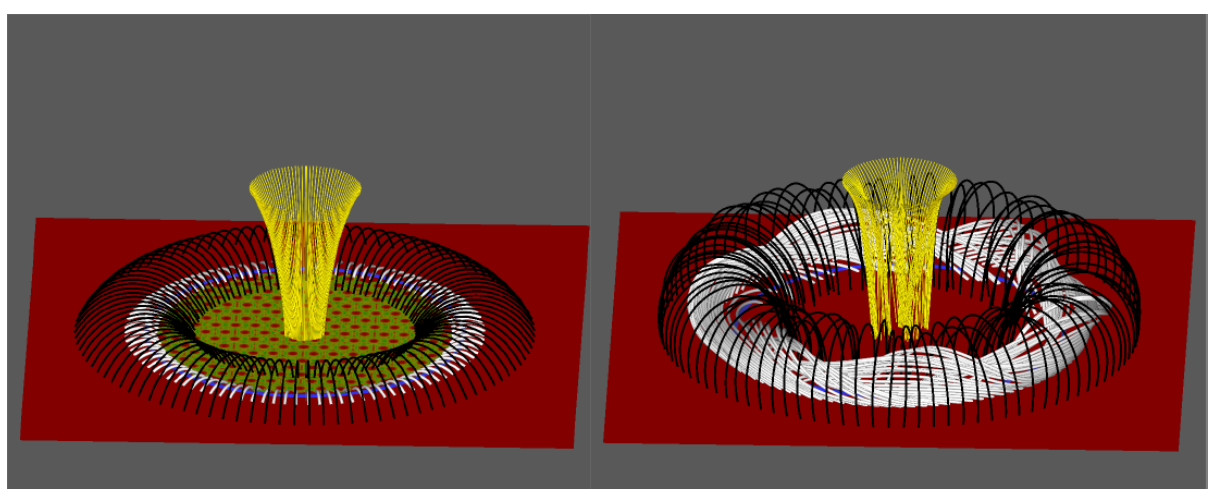

Fig. 8 Initial (left) and final (right) states of a coronal system driven by random small-scale helicity and free energy injection. The blue circle indicates the PIL and the contours inside this PIL in the left panel show the flow pattern at the photosphere. The pattern is rotated randomly after a half rotation (From Knizhnik et al 2017). (C)AAS. Reproduced with permission.

though most of the helicity and free energy has been injected into this region. The white field lines near the PIL, on the other hand, are all highly sheared along the PIL. Note that the shear is highly localized about the PIL, as in observations (e.g. Schmieder et al, 1996) and exactly as required for a filament channel. Furthermore the black lines, although pushed upward by the increased magnetic pressure of the filament channel, are smooth and laminar, as observed for coronal loops (Schrijver et al, 1999).

Previous studies discuss the buildup of shear flows due to a significant Lorentz tension force. This is due to the non-neutralized currents developed exclusively along strong PILs (Georgoulis et al, 2012a), verified independently by Török et al (2014b), although the latter study does not necessarily attribute net currents to the Lorentz force. The entire process leading to Lorentz force-driven shear is ultimately fueled by flux emergence (Manchester et al, 2004). Shear flows largely cease when flux emergence is complete, even at established PILs.

A key point evident from Figure 8 is that the FC field lines show no evidence for a coherent global twist. The structure is clearly that of a SMA rather than a MFR. For a SMA, the helicity in the system is primarily due to the mutual linkages between the strongly sheared FC field and the overlying quasi-potential flux. Figure 8 is a dramatic demonstration of how reconnection can convert one form of geometrical linkage to another. The helicity injected into the system was all due to small rotations, in other words, the internal twist of many small flux tubes. Reconnection, however, converts this helicity into a global shear. But we caution the reader that inverse cascading of helicity does not necessarily lead to shear as shown amply, for example, by studies of the kink instability. In addition, a strongly stressed PIL is required to obtain substantial shear (see also the theoretical work by Alexakis et al, 2006). We also note that the final system in Figure 8 is not in a minimum energy linear force-free state. The reason is that the line-tying introduces additional constraints on the system evolution beyond only global helicity conservation (Antiochos, 2013).

A simple schematic as to why coronal helicity condenses onto the PIL is presented in the Figure 9. Since the photospheric driving speeds, $\sim 1 \mathrm{~km} / \mathrm{s}$, are much 
smaller than the coronal Alfvén speed, and the corona is low $\beta, \sim 10^{-2}$, the coronal evolution is approximately given by a quasi-static sequence of force-free states. In that case the twist field i.e., the azimuthal field component is constant along a twisted flux tube, neglecting the effect of flux-tube expansion, and consequently, the evolution can be captured by considering only the twist field projected onto the 2D photospheric surface. Figure 9 shows that reconnection between any two neighboring twisted flux tubes results in the spreading of the twist field so that it encompasses the flux of both tubes. Further reconnection of these larger tubes spreads the twist flux further until it "condenses" against the PIL, because this boundary defines the extent of the flux system. This shows that inverse cascading within the PIL flux system is at the heart of the helicity condensation model with reconnection being a key process here. Rigorous numerical calculations, such as the one shown in Figure 8 above (e.g., Knizhnik et al, 2015, 2017; Zhao et al, 2015), have confirmed both the basic picture of Figure 9 and the estimates for the rate of FC formation given in Antiochos (2013).

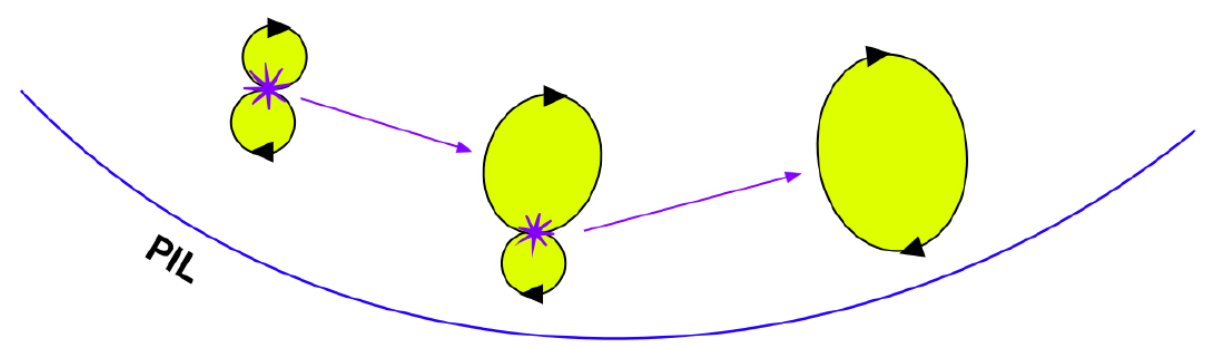

Fig. 9 Schematic drawing of the evolution giving rise to the results of Figure 8 The yellow circles indicate the photospheric footpoints of twisted coronal flux tubes. Reconnection of the twist component of the two elemental flux tubes on the left, which were formed by photospheric motions, causes them to merge and form the larger twisted flux tube in the center. Reconnection of this flux tube with another elemental flux tube produces the large flux tube on the right. Note that this process successively moves magnetic stress toward the PIL where it appears as a large-scale coherent shear.

\subsubsection{Theoretical Issues}

At least two important theoretical issues remain to be addressed by the helicitycondensation model. The first is the effect of a multi-scale photospheric driving. The calculations to date used a single scale for both the helicity injection and the small-scale driving that powers the turbulent reconnection, but in the corona these are likely to occur at different scales.

The second critical theoretical issue is the effect of flux cancellation on helicity condensation. In the Knizhnik et al (2017) simulation, the PIL itself was not driven in order to avoid the effects of flux cancellation, but there is no doubt that flux does cancel at PILs. As originally described by van Ballegooijen and Martens (1989), and subsequently modeled by many authors (e.g., Amari et al, 2000, Mackay et al, 2010) flux cancellation occurred systematically along a PIL in a quasi-2D evolution. In this classic scenario, flux cancellation at the photosphere is physically similar to flare reconnection along a quasi-2D X-line. Both simulations and observations 
have shown that such quasi-2D reconnection inevitably produces a near potential arcade below the reconnection line (the flare loops) and a large-scale MFR with global systematic twist above the line (the CME "plasmoid").

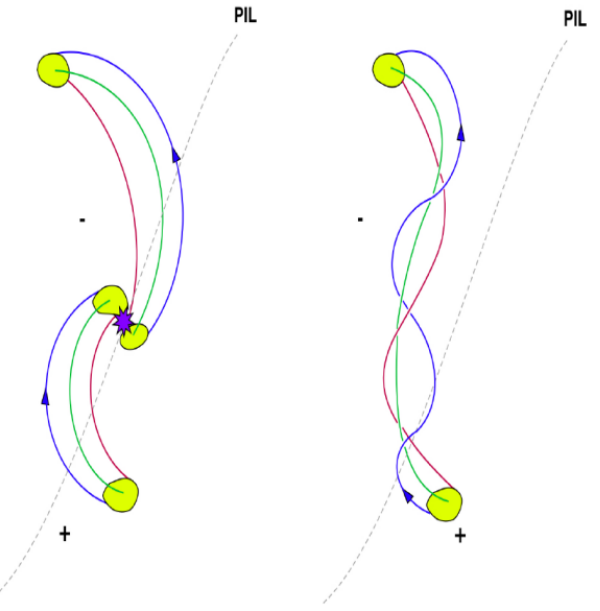

Fig. 10 Schematic drawing of realistic 3D flux cancellation at a large-scale photospheric PIL. Shown on the left are four photospheric flux concentrations (yellow patches) that are the footpoints of two coronal flux tubes. Originally all four concentrations were far from the PIL, but photospheric motions bring two opposite polarity concentrations to the PIL where they cancel by reconnection and submergence. This cancellation results in the single coronal flux tube shown on the right, which is twisted and has both footpoints far from the PIL.

Observations show, however, that except possibly at the centers of young ARs where flux has just emerged, flux cancellation is far from systematic, and is instead a quasi-random fully 3D process. High-resolution observations of the photospheric magnetic field show that cancellation consists of two strong concentrations of opposite polarity flux ("magnetic elements"), seemingly randomly colliding and disappearing (Berger and Title, 1996). Figure 10 illustrates the nature of flux cancellation given the observed photospheric flux evolution. Two concentrations of opposite polarity flux are very near a large-scale PIL, where they collide and cancel by photospheric motions. The other ends of the two flux tubes are not equally near to the PIL, as in the systematic cancellation models, but instead are much further away. Even this picture is a gross simplification. In the actual corona, the concentrations near the PIL would connect to several concentrations away from the PIL, and there is always weak flux between the concentrations (e.g., Wiegelmann et al, 2014, Cheng and Ding, 2016).

The implication of Figure 10 above is that flux cancellation is simply another source of small-scale twist injection into coronal loops, just like convective flows and flux emergence. This small-scale twist, along with the twist from the other sources, then undergoes the helicity cascade process and ends up as a large-scale PIL shear. This small-scale twist, along with the twist from the other sources, then undergoes the helicity cascade process and ends up as a large-scale PIL shear. Rigorous MHD simulations are now required to test this hypothesis in tandem with 
observational studies aiming to fully characterize the properties of flux cancellation on both small and large spatial scales.

\subsubsection{Observations of magnetic helicity}

Helicity exhibits inverse cascade to shorter wavenumbers, a fact well-established from theory and simulations (Alexakis et al, 2006). However, most pieces of evidence about its consequences in the solar atmosphere are qualitative. Apart from the concentration of shear almost exclusively around flux-massive PIL areas (e.g., Hagyard et al, 1984; Moore et al, 1987, Zirin and Liggett, 1987, Tanaka, 1991, Schrijver, 2007), indirect evidence for the inverse cascade of helicity is provided by the conversion of some of the twist of MFRs to writhe (a measure of the deformation of the MFR axis; see, e.g., Török et al, 2010) in the course of the helical kink instability, which has a threshold that is reached at around a twist of $N=1.25$ or more (e.g., Hood and Priest, 1981, Török et al, 2004). We note that in the line-tied corona the kink by itself could not move helicity across field lines, but it would enhance the possibility of reconnection, which then transports helicity. Furthermore the amount of writhe produced depends primarily on the evolution of the instability, not just on the initial twist (Török et al, 2014a). A typical observed example of a helical kink instability can be found in Rust and LaBonte (2005). Moreover, the inverse cascade of helicity can be invoked to interpret the gradual mutual-toself helicity conversion pattern reported by Tziotziou et al (2013) (Figure 11) in an emerging eruptive AR (see also Li et al, 2014 , for another example). Inverse helicity cascading has been quantified in phase space by Zhang et al $(2014,2016)$, who found that the unsigned current helicity spectrum, $H c(k, t)$, of two ARs show a $k^{-5 / 3}$ power law. They also found that the current helicity spectrum is related to the magnetic helicity spectrum, $H m(k, t)$, via $\left.H c(k, t) \simeq k^{2} H m(k, t)\right)$. This result is consistent with simulations of hydromagnetic turbulence (e.g., Brandenburg and Subramanian, 2005) and implies that turbulence becomes gradually less helical towards smaller scales. The same authors studied the solar cycle evolution of helicity spectra and found that helicity spectra steepen as we progress to solar maximum, emphasizing the large-scale magnetic field.

Indirect observational evidence in support of the helicity-condensation model comes from the studies of filament chirality (e.g., Mackay and van Ballegooijen, 2005, Yeates and Mackay, 2012). These authors found that the standard processes of differential rotation, AR emergence, flux diffusion and cancellation, etc. could not reproduce the observed global distribution of filament chirality and eruption rate, especially during the declining phase of a solar cycle. The implication from these studies is that an additional mechanism is needed for injecting helicity into the corona and concentrating it along PILs. The helicity condensation model provides such a mechanism (Mackay et al, 2018).

Several observations support the important role of helicity in the initiation of eruptions (see Pevtsov et al 2014 for details). Nindos and Andrews (2004) found that, statistically, the pre-flare absolute value of the linear force-free field parameter and the resulting coronal helicity are larger in ARs that produce eruptive major flares than in those that produce major but confined flares. A similar conclusion was reached by LaBonte et al (2007) who calculated the helicity flux in large samples of X-flaring and non-X-flaring ARs and found that a necessary condition 


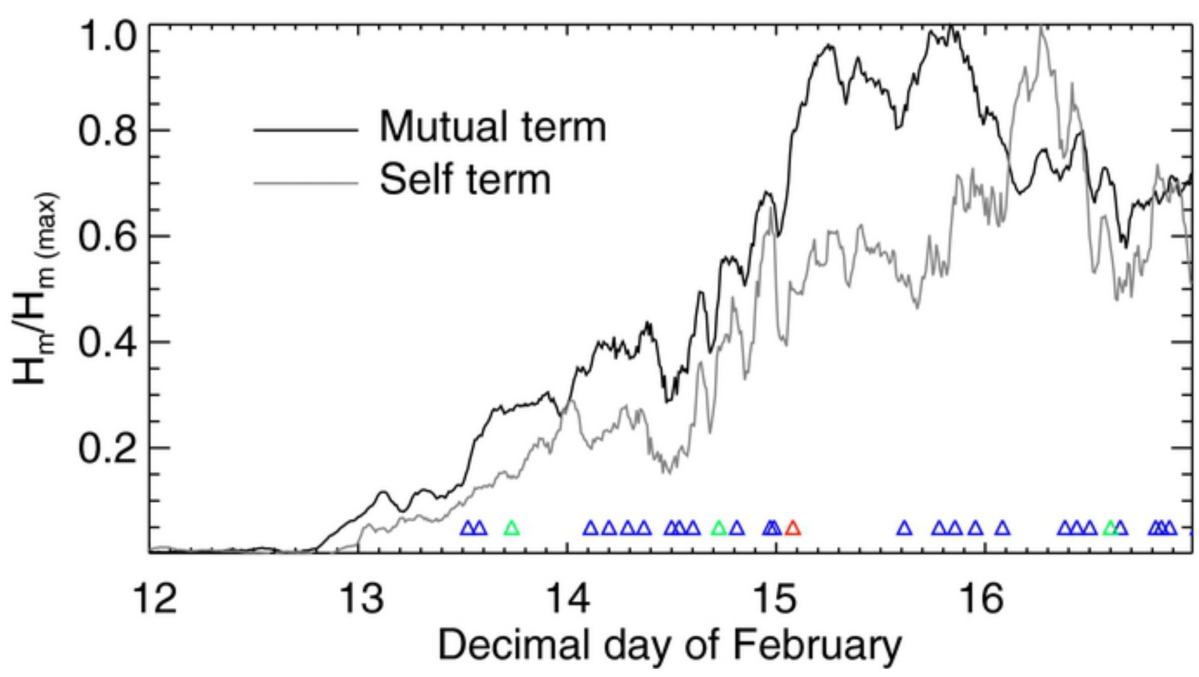

Fig. 11 An example of mutual-to-self relative magnetic helicity transformation during the emerging-flux phase in observed NOAA AR 11158, primarily along the AR's strong PIL. The self-helicity term (gray curve) follows closely the mutual-helicity one (black curve), lagging from it by a few to $\sim 24$ hours. The triangles adjacent to the time axis correspond to onset times of flaring activity in the AR. Modified from Tziotziou et al., (2013). C)AAS. Reproduced with permission.

for the occurrence of X-class flares is that the peak helicity flux has a magnitude $>6 \times 10^{36} \mathrm{Mx}^{2} \mathrm{~s}^{-1}$. Park et al $(2008,2010)$ studied about a dozen X-class flares and found that they were preceded by a significant helicity accumulation, $(1.8-32) \times 10^{42} \mathrm{Mx}^{2}$, over periods of half a day to a few days.

Using the method developed by Georgoulis et al (2012b), Tziotziou et al (2012) calculated the instantaneous relative helicity and free magnetic energy of several ARs and found a significant monotonic correlation between the free energy and the relative helicity. This correlation reinforces the notion that, in addition to free energy, helicity may play a central role in solar eruptions. They also found that the eruptive ARs were well segregated from the non-eruptive ones, in both free energy and helicity, with free energy and helicity thresholds for the occurrence of major (GOES M-class and above) flares of $4 \times 10^{31} \mathrm{erg}$ and $2 \times 10^{42} \mathrm{Mx}^{2}$, respectively. Nindos et al (2012) found that the initiation of eruptions in a large emerging AR depended critically on the accumulation of both free energy and helicity in the corona and not on the temporal evolution of the variation of the background field with height.

Pariat et al (2017) performed eruptive and non-eruptive 3D MHD simulations in which they decomposed the relative helicity, $H$, into a term $H_{j}$ associated with the current-carrying magnetic field and a term $H_{p j}$ associated with the intrahelicity between potential and current-carrying fields. They found that the ratio $H_{j} / H$ yields a reliable eruptivity proxy. It will be interesting to check the performance of $H_{j} / H$ against observations, as well, although one will ultimately need to rely on a model or extrapolation for the unknown coronal magnetic field. The above results highlight the importance of helicity buildup in the initiation of eruptions. Along with other observational and modeling results, reviewed by Georgoulis et al 
(2019), such findings place magnetic helicity on equal footing with free magnetic energy in terms of their roles in the initiation of solar eruptive events.

\section{The Magnetic Configuration of Filament Channels}

We now discuss the theoretical expectations and observational signatures used in interpreting the FC magnetic configurations as SMAs or MFRs. Although we describe the two types as separate subsections, we contrast the SMA and MFR interpretations throughout. To make the discussion easier to follow, we organize the text according to the various signatures (e.g. prominence dips, sigmoids, cavities, etc.), given in italics at the beginning of the relevant paragraph.

\subsection{Sheared Magnetic Arcades (SMAs)}

\subsubsection{Introduction}

Early attempts of modeling the magnetic structure of filaments or prominences were based on $2 \mathrm{D}$ or $2.5 \mathrm{D}$ (translationally invariant) arcade configurations. None could account for the existence of plasma-supporting magnetic dips. On the one hand, bipolar 2.5D force-free arcades were unable to generate magnetic dips (see Klimchuk, 1990, Amari et al, 1991), which were considered necessary to support filament material. On the other hand, MHD models had difficulty in forming low- $\beta$ and stable weight-induced dips (see e.g. Wu et al, 1990; Choe and Lee, 1992). So SMAs with dips, if they ever existed, had to be intrinsically 3D, unlike MFRs that already have dips in $2.5 \mathrm{D}$. This was not only a geometrical challenge, but also a computational one, given the limitations in the early 90's. A second challenge was on the observational side. Magnetic field measurements within prominences were showing a vast majority of so-called inverse-polarity (IP) configurations (Leroy et al, 1984, Bommier and Leroy, 1998). In these configurations, the horizontal field viewed from above points toward the opposite direction than the regular normal-polarity (NP), i.e., it goes from the negative toward the positive polarity. While this peculiar behavior is readily satisfied in the windings of MFRs, it was not obvious how this could be achieved in mere bipolar arcades, which are naturally NP (Klimchuk et al, 1988).

All these challenges were simultaneously solved in Antiochos et al (1994) in terms of a fully 3D model leading to formation of force-free and inverse-polarity dips in the SMA models. The computational limitations were overcome by using the original magneto-frictional approach from Yang et al (1986) instead of a full-MHD model. The results were later confirmed by MHD modeling (DeVore and Antiochos 2000; Aulanier et al 2002, Figure 12). The geometrical issues were simplified to the extreme by concentrating surface-motion-induced magnetic shear close to and around the PIL, also satisfying observational constraints of strong shear being confined to PILs (Schmieder et al, 1996). It was found that when the extension across the PIL of the bipolar field is smaller than the length of sheared field lines along the PIL, then IP-dips are naturally produced (see Figure 13).

The first key result of this investigation was that the stretching of the sheared field lines away from these strong-field regions leads to their horizontal expansion 

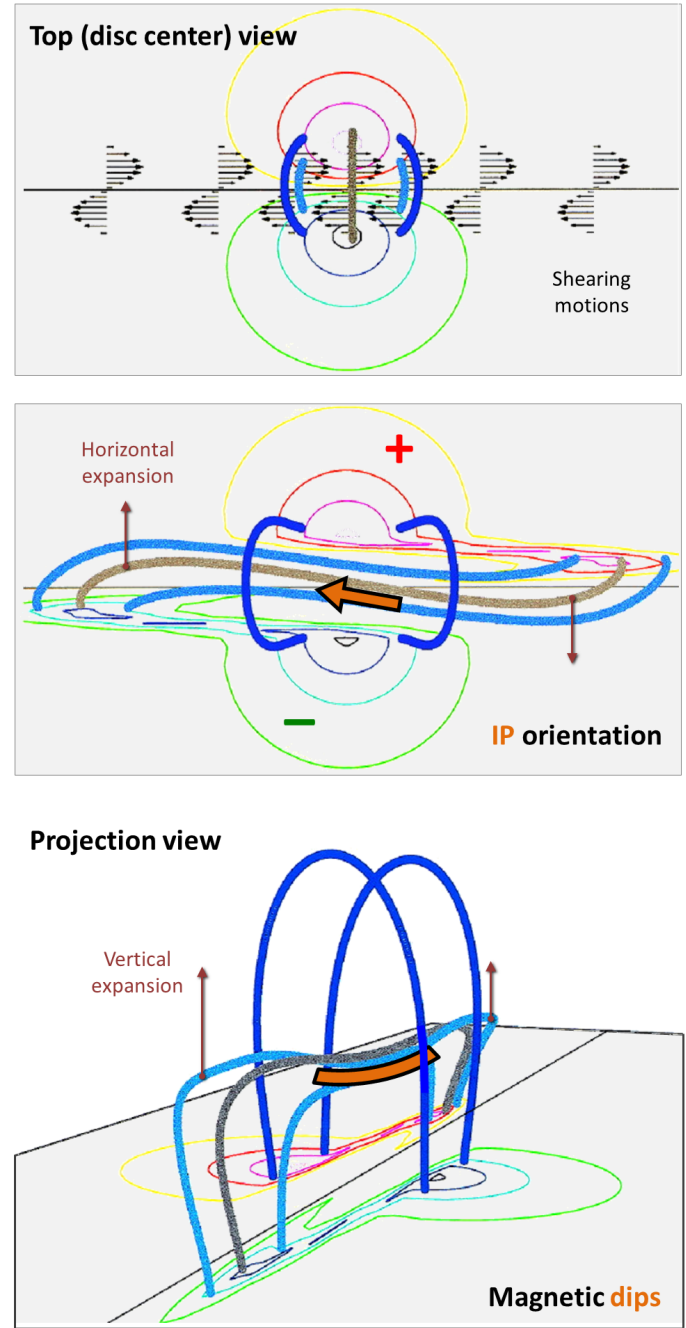

Fig. 12 Overhead views of the initial condition (upper panel) and of the final state (middle panel) of a SMA simulation. Perspective view of the final state of the same simulation (lower panel). The colored contours on the bottom of each panel correspond to photospheric Bzcontours while the thick colored lines correspond to magnetic field lines. Modified from DeVore and Antiochos (2000). (C)AAS. Reproduced with permission.

towards weaker fields, i.e., towards the opposite side of the PIL. This produces S-shaped (sigmoidal) field lines with an IP orientation at their center. The second key result was that the vertical expansion of these sheared arcades is larger at large distances along the PIL than it is within the core of the strong bipolar fields, owing to stronger magnetic tension in the bipole center. This produces a dipped geometry at the center of the field lines, right where an IP orientation is present.

The SMA model was also extended to interacting bipoles aligned along the PIL (DeVore et al, 2005, Aulanier et al, 2006a). When the appropriate conditions are met, as far as observational constraints on magnetic helicity and prominence merg- 
ing are concerned (see Aulanier and Schmieder, 2008), then the sheared bipoles reconnect with each other. We note that this reconnection takes place in the socalled finite- $B$ slip-running reconnection regime, i.e., occurring at regions of drastic changes in the magnetic field gradient, and does not abruptly change field-line connectivity (Aulanier et al, 2006b). Reconnection between sheared bipoles merely produces a longer SMA, with an inhomogeneous spatial distribution of dips that can support prominence plasma, as observed outside of ARs where the surface magnetic field is not concentrated in a single bipole. Therefore, the SMA model can in principle account also for large quiescent prominences, either between or within decaying ARs, or in the polar crowns.

The SMA model may be criticized because of its somewhat artificial distribution of flux at the Sun's surface, which results from the shear-driven elongation of the bipole along the PIL. While this issue remains to be addressed in single-bipole AR SMA models, solutions have been proposed in the framework of multi-bipole quiescent-prominence SMA models (see below) and of the helicity-condensation model (see Section 2.3). Nevertheless, the SMA models naturally provide a viable and straightforward model for the magnetic structure of FCs, i.e., for pre-eruptive magnetic configurations.

\subsubsection{Pre-eruption signatures of SMAs}

Magnetic Field Dips: In the related MHD calculations, SMAs dominate the core of the PIL. Also, the modeled field-line dips are always dominated by IP-dips, irrespective of the shear magnitude. In addition, a key property is that all SMA models discussed here possess narrow regions of NP dips at their top, within, and at the edge of the strong surface fields (bottom left panel of Figure 13). When it was identified, this property was proposed to be a clear-cut discriminator between SMA and MFR. This was disputed, however, when it was found that MFRs can also produce NP dips at their center, when they were perturbed by small-scale surface flux concentrations in the vicinity of a fragmented PIL (Aulanier and Démoulin, 2003). Such small polarities hardly exist in the core of young solar ARs with $\delta$-spots e.g., Fig. 17 in van Driel-Gesztelyi and Green (2015), from which the most energetic eruptions originate (e.g., the review of Toriumi and Wang, 2019) . Therefore, identifying NP dips in compact AR sigmoids and filaments may provide a method for discriminating between the SMA and the MFR model.

SMA models tend to produce rather shallow dips. This comes naturally from differential shearing, which makes the SMAs expand differentially in the horizontal and the vertical direction in the corona. Calculations of the formation and evolution of prominence condensations were achieved in such shallow dips, within the thermal non-equilibrium model (Antiochos and Klimchuk, 1991). The calculations were done for generic shallow dips as well as nearly-flat field lines (Antiochos et al, 1999, 2000, Karpen et al, 2001, 2006), and also using field line geometries that directly result from MHD simulations of single and double-dipole SMA models (Karpen et al, 2006, Luna et al, 2012). A key property of SMA shallow dips (as well as their surrounding nearly-flat field lines) is that they permanently host very dynamic prominence condensations. This behavior is observed in many filaments (Schmieder et al, 1991, 2017; Lin et al, 2003, 2012). This does not hold for highly twisted MFRs, where deep dips are produced by the large-scale winding of coronal field lines. In the SMA models, only the lower dips are deep enough to prevent a 

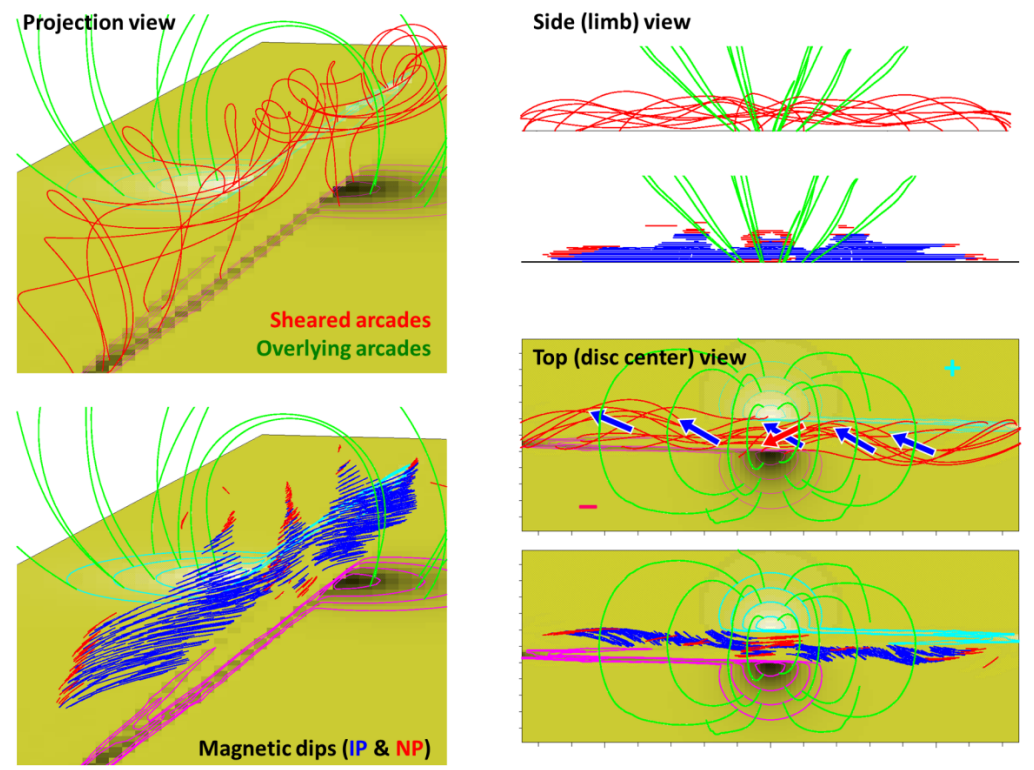

Fig. 13 Projection view of a SMA from a fully 3D MHD simulation applying large shears in the photosphere; SMA (red) and overlying arcade (green) field lines (upper left panel); distribution of IP (blue) and NP (red) dips (lower left panel). Side (limb) and top (disk center) views with the same same format as the projections views. Modified from Aulanier et al (2002). (C)AAS. Reproduced with permission.

dynamic behavior. It has therefore been argued that this property is another possible discriminator between the SMA and the MFR models. In addition, shallow dips are also implied by the observation of linear threads in high spatial resolution images of prominences and filaments. (e.g., Okamoto et al, 2007; Vourlidas et al, 2010.

Note that the dips lose their discriminating characteristic for weakly to moderately twisted MFRs i.e., up to two turns or so. In that case, the dips in the upper part of the MFR are shallow too (see, e.g., Figure 2e in Gibson and Fan, 2006a), leading to dynamic plasma condensations there (Xia et al | 2014, Xia and Keppens, 2016), and stable condensations in the lower part of an MFR, just as for an SMA. The above discussion suggest that the dynamic behavior of prominence plasmas rules out highly twisted MFRs as viable models for quiescent prominences and hence, more generally, as CME progenitors.

One has to take into account, though, that prominence models based on the distribution of field-line dips (e.g., Aulanier et al, 1999, 2002, Lionello et al, 2002, van Ballegooijen, 2004, Dudík et al, 2008, Zuccarello et al, 2016), as well as MHD simulations of prominence formation in MFR configurations (Xia et al, 2014; Kaneko and Yokoyama, 2018, Fan, 2018) suggest that prominence plasma is often organized in a vertical, sheet-like structure, as the observations suggest for quiescent prominences. In such cases, prominence threads would occupy only relatively short sections of twisted field lines (since such field lines cross the sheet rather than running along it; see, e.g., Fig. 3 in Xia and Keppens, 2016), rendering the display of twist in quiescent prominences improbable. 
Moreover, some FCs may consist of so-called 'hollow-core' MFRs, in which twisted field lines exist only at the periphery of the configuration, while the core field lines run more or less parallel (e.g., Bobra et al, 2008, Titov et al, 2014, see also Fig. 14). Thus, the fact prominence threads very rarely show indications of twist does not exclude that the underlying structure is an MFR.

Magnetic Field Topology: Topology is a fundamental difference between SMAs and MFRs. MFRs are separated from the surrounding coronal arcades. In 3D, this requires either a coronal separatrix surface, which emanates from a bald patch (BP; Titov et al, 1993) along the photospheric PIL or by quasi-separatrix layer (QSL), whose core is a hyperbolic flux tube (HFT Titov et al, 2002) that displays an $\mathrm{X}$-shape when viewed along the PIL (Section 3.2). An example of an HFT is given in Panel (a) of Figure 14. This Figure contains maps of the force-free parameter $\alpha$ ( $\propto$ the parallel electric current), and as shown by Aulanier et al (2005), coronal currents maps trace QSLs. Pure SMAs possess neither of these topological features.

Instead, they exhibit a gradual continuous transition from the sheared core to the current-free overlying arcades. This "coronal magnetic shear gradient" is a direct consequence of the prescribed smooth shearing profile at the solar surface (see Figure 12. We note that, under non-ideal MHD conditions, transient, low coronal dips could potentially appear in SMAs, during SMA merging. These dips occur along short PIL sections and do not reach all the way to the photosphere, so they will not appear as "traditional" BPs (Aulanier et al, 2006a). They are expected to be rare. However, with the increased availability of chromospheric vector magnetic field measurements, it is possible they could be observed as 'chromospheric' BPs. We believe that the investigation of photospheric versus chromospheric BPs in high resolution magnetograms in multiple atmospheric layers could be a fruitful discriminator between MFRs and SMAs in the near future.
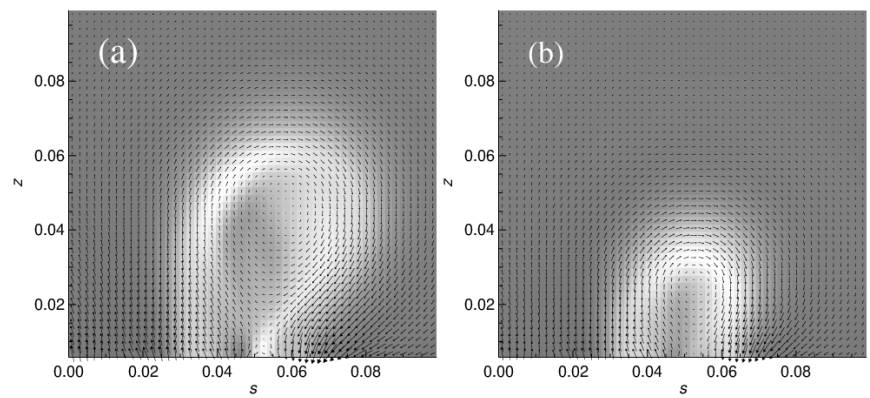

Fig. 14 Force-free parameter $\alpha$ (gray scale) and in-plane magnetic field vectors (arrows) in a vertical plane perpendicular to the magnetic axis of two numerically relaxed NLFFF models of an AR-FC, constructed using the flux rope insertion method. In (a), a higher amount of axial (i.e., highly sheared) flux was inserted, resulting in a so-called hollow-core MFR (Section 3.2.1, which features an HFT at its underside and is slightly unstable. In (b), the lower amount of axial flux results in a stable hybrid configuration, close to a hollow-core MFR, which neither contains an HFT nor a BP. Modified from Kliem et al (2013). C)AAS. Reproduced with permission. 


\subsubsection{Observational Signatures of Eruptions from SMAs}

We now turn to observational interpretations in favor of SMAs. Two models are mainly employed to explain how an SMA can erupt. The tether-cutting model (e.g. Moore and Roumeliotis, 1992, Moore et al, 2001, Priest et al, 2016) is based on the assumption that magnetic reconnection between two loop-like sheared arcades within the AR core field creates an MFR and drives the eruption. The second approach, known as the "breakout model" (Antiochos et al, 1999), postulates that shearing motions drive the expansion of a core SMA, which may erupt only after it breaks through the overlying restraining fields via reconnection at a magnetic null point.

'Tether-cutting' vs. Breakout: There are important differences between the two models: (i) tether-cutting can occur in either a bipolar or multipolar configuration, whereas the breakout model requires a multipolar magnetic configuration, and (ii) both models predict pre-eruption brightenings but at different locations. For tether-cutting the brightenings occur at the AR core while for the breakout model they occur at remote locations (and may be fainter). These two major differences are often used to postulate the pre-eruption magnetic configuration. We note that breakout could also trigger the eruption of a pre-existing MFR but in such a case the timing between flare and breakout reconnection may much tighter than in the case of "standard" breakout.

Crinkles: In a series of studies, Sterling et al. investigated the formation and distribution of transient small-scale brightenings observed in UV spectral lines away from the core of the AR, which they called crinkles. Sterling and Moore (2001a), Sterling et al (2001), and Sterling and Moore (2004) found that the crinkles occurred before the core brightenings. This timing is consistent with the breakout model, which advocates the eruption of an SMA. It can be argued, though, that a pre-existing MFR in a quadrupolar configuration may become unstable even without involving breakout reconnection (e.g., Amari et al, 2007), and that such an eruption may produce crinkles in a similar way. Also, the eruption of a pre-existing MFR may be triggered by breakout.

Chen et al (2016) presented a rare case, where AIA $94 \AA$ loops are seen to form a nearly ideal quadrupolar breakout configuration, followed by a CME and an X-class long-duration flare. While the external reconnection events (crinkles) do suggest that initiation of an eruption may take place without involving internal reconnection, they, however, do not allow to unambiguously discriminate between eruption of an MFR and that of an SMA. Therefore, we suggest that the timing and location of EUV brightenings may say something about the trigger mechanism, but possibly nothing about the nature of the pre-eruptive configuration.

Reconnection between erupting and surrounding fields may complicate the interpretation. Sterling and Moore (2001b) reported an event that obviously started from internal reconnection followed by the ejection of the core field, which later reconnected with external overlying fields, leading to the appearance of crinkles. Joshi et al (2017) analyzed a "three-ribbon" flare and concluded that first an MFR formed and erupted following the tether-cutting scenario, and then the erupting fields reconnected with large-scale enveloping fields, which led to signatures similar to those expected from the breakout mechanism. If this scenario is common it will make it difficult to say whether breakout reconnection was the cause or just a re- 
sult of an eruption, unless there is a clear time difference between the appearance of crinkles and flare loops.

\subsection{Magnetic Flux Ropes (MFRs)}

In Section 2, we discussed three mechanisms that can generate MFRs; namely, photospheric flux cancellation, reconnection in the lower solar atmosphere between pre-existing and emerging flux or between pre-existing flux systems, and bodily emergence of an MFR. We now review the theoretical expectations (Sec 3.2.1) and observational signatures (Sec. 3.2.2) suggested so far in the literature.

\subsubsection{Theoretical Expectations}

Since magnetic twist is a major element of MFRs, it is important to consider the expected number of turns in pre-eruptive MFRs. This can be derived from a number of theoretical and modeling considerations. First, since an MFR arches upward from its photospheric footprints, it will contain dipped field lines only if its twist reaches a (weakly geometry-dependent) minimum value, unless the MFR has a straight horizontal section. The schematic in Figure 15 illustrates that dips tend to form first at the bottom edge of the rope under the apex and require about one field-line turn if the rope arches upward (see Priest et al 1989 for the detailed geometric dependence).

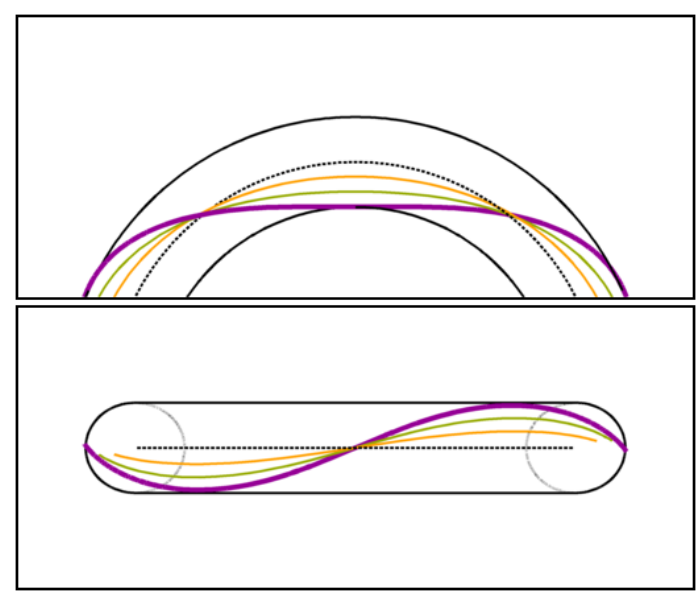

Fig. 15 Side and top view of an MFR of the minimum twist number that just yields a dip of a field line in the MFR. This tends to be the field line at the bottom edge under the apex (shown in dark violet) for a symmetric MFR whose twist profile is not strongly peaked at the axis. The minimum twist number is close to unity, $N \approx 1$, and only weakly dependent upon the geometry of the MFR (Priest et al 1989).

Second, a twist slightly exceeding one turn typically results when the inner part of an SMA is transformed into an MFR by reconnection under the rope axis. Since the reconnected flux passes over both legs of the arched magnetic axis 
and under the axis at or near its apex, it makes exactly one turn between the points where it passes over the axis; the remaining sections of the reconnected flux, down to its footprints, add a relatively small fraction of one turn to the total twist (see the schematic in Figure 15). The formation or enhancement of MFRs by flux cancellation (van Ballegooijen and Martens, 1989, Aulanier et al, 2010, Green et al, 2011; Zuccarello et al, 2015), during eruptions (Lin et al, 2004: Patsourakos et al 2013), and even in many flux-emergence simulations (Manchester et al, 2004 Archontis and Hood, 2012, Leake et al, 2013, Syntelis et al, 2017) involves such reconnection, so that MFRs associated with FCs and eruptions are likely to possess a twist slightly above one turn.

Third, arched MFRs without field-line dips, i.e., of less than one field-line turn, visually resemble an SMA. Such ropes are unlikely to be inferred in images of coronal structures that outline field lines, i.e., from prominence threads or EUV/SXR sigmoids.

Fourth, NLFFF models for FCs and their environment in the corona can be obtained through extrapolation of a vector magnetogram (e.g., Wiegelmann, 2008), magneto-frictional relaxation (e.g., van Ballegooijen et al, 2000), through the flux rope insertion method (van Ballegooijen, 2004), as well as through evolutionary methods (Yeates and Mackay, 2009, Mackay et al, 2011; Yardley et al, 2018b; Chintzoglou et al, 2019|| Price et al, 2019). The cross-section average of the inferred twist ranges from below unity to $N \approx 2$. Using extrapolation, an MFR was found in a number of cases (e.g., Canou et al, 2009, Yelles Chaouche et al, 2012, Guo et al, 2013: Cheng et al, 2014b; Chintzoglou et al, 2015; Wang et al, 2015: Liu et al, 2016, Zhao et al, 2016, Green et al, 2017; James et al, 2018, Mitra et al, 2018, Duan et al, 2019, Woods et al, 2020), while an SMA resulted in others (e.g., Sun et al, 2012).

For some ARs, one extrapolation technique found an MFR and others found an SMA (Schrijver et al, 2008, Inoue et al, 2016, Jiang et al, 2016, Amari et al, 2018). The experience with this technique over many years indicates that it often struggles to find an MFR (e.g., Schrijver et al, 2008) and that success in finding one is more reliable than failure. Success in finding an MFR is corroborated by the presence of bald patches in the filament channel in most cases.

Fifth, the excitation of the helical kink instability, a possible mechanism of eruption onset, requires a twist of at least $N>1.25$ in the current-carrying cross section of the MFR (Hood and Priest, 1981), but more likely $N \gtrsim 1.5-1.75$ in realistic configurations with a guide field component (Török et al, 2004, Fan and Gibson, 2004). Therefore, pre-eruptive MFRs with high twist $(N>2)$, if they exist, should not be stable for long intervals, except under certain conditions such as a strong guide field or a very thin MFR (see the discussion in Török et al, 2014a). This suggests that most pre-eruptive, stable MFRs should be weakly to moderately twisted, within the range of $N \approx 1-2$ turns.

Another important aspect is the "visibility" of the twist and, related to this, the radial twist profile. An MFR can have any radial twist profile, $N(r)$. Cases with $N(r)$ peaking at the axis $(r \rightarrow 0)$ have been obtained in simulations of flux emergence (e.g., Fig. 3b in Török et al, 2014b). So-called hollow-core MFRs (see also Section 3.1.2), whose twist profile peaks at the surface $(r \rightarrow a)$, have been found to yield good models of FCs in decaying ARs which are dominated by flux cancellation (e.g., Bobra et al 2008; Savcheva and van Ballegooijen 2009, Su et al 2011. Figure 14. Especially the latter models have received widespread attention 
in the modeling of FCs. They consist of highly sheared but (possibly very) weakly twisted flux in the core, surrounded by a layer of clearly (but not necessarily highly) twisted flux at the surface of the structure. As a whole, the structure is an MFR, although the non-potentiality of the core flux is strongly dominated by shear. The field lines in the core flux obviously display very small twist (e.g., Savcheva and van Ballegooijen, 2009), and the same can be expected of field line tracers in solar observations. However, the latter is a difficulty for a much wider range of MFRs, except those particularly highly twisted around the axis. Any twist near the MFR axis is much less visible than twist of the same magnitude near the surface. See illustrations of this phenomenon for a uniformly twisted MFR, e.g., in Priest (2014, Figure 3.5) and Török et al (2010, Figure 2).

\subsubsection{Observational Signatures}

Given that several of the proposed MFR tracers apply uniquely to either QS or AR filament channels, we discuss them separately below. We attempt to draw parallels between them, where applicable.

\section{MFR Signatures in QS Filament Channels}

The model for a filament channel hosting a quiescent prominence is given by an MFR in many studies (Kuperus and Raadu, 1974, van Ballegooijen and Martens, 1989, Priest et al, 1989, Low and Hundhausen, 1995: Aulanier and Démoulin, 1998 , see Section 3.1 for SMA models). This is based, first of all, on the observation that most quiescent prominences exhibit inverse polarity (Leroy et al, 1983, 1984, Bommier et al, 1994, Hanaoka and Sakurai, 2017). Inverse polarity results naturally if the prominence material is trapped in the magnetic dips in the lower half of an MFR, but requires special conditions if the prominence material is trapped in an SMA (see below).

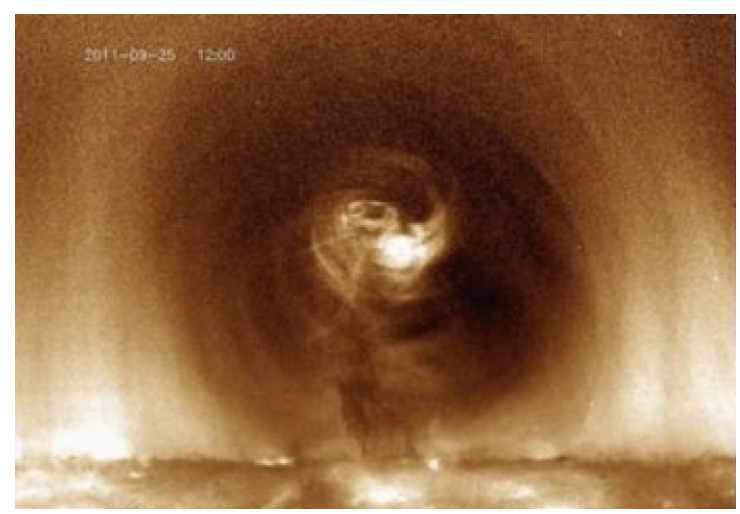

Fig. 16 Quiescent prominence and its coronal cavity observed by AIA in the $193 \AA$ channel (from Li et al 2012). CAAS. Reproduced with permission.

Magnetic Field Dips: Inverse polarity dips are possible in an SMA if its end sections lean over the PIL and bulge upward as discussed in Section 3.1.2. This happens if the main flux sources on either side of the PIL are considerably displaced along the PIL, which is possible for a strongly sheared region. However, for the 
effect to be significant, the displacement must be such that the middle section of symmetric and strong confinement must be relatively short compared to the length of the SMA. Otherwise, the SMA field lines will be closely aligned with the PIL in the middle section, so that the inverse field component will be much weaker than the axial component, i.e., it will not reach the observed angle of 10-30 degrees from the PIL (e.g., Hanaoka and Sakurai, 2017). While the displacement of the main flux sources may satisfy this requirement for some ARs and intermediate filament channels, it seems unlikely to be realized for the filament channels of long quiescent prominences, which can reach a solar radius in length or more. This obviously contrasts the arguments in Sec. 3.1.2 regarding the ability of SMA models to contain IP dips. Detailed, quantitative observations of shear patterns in the photosphere are required to test these assertions.

Thread Orientation: The typical orientation of the threads relative to the axial field of quiescent filaments, combined with the assumptions of force-free equilibrium and the presence of a net current along the filament, is consistent with an MFR or the MFR component in a hybrid structure only. This results from the following reasoning. The field lines in a right-handed SMA and in the top half of a right-handed MFR point to the right relative to the direction of the axial field of the prominence in vertical projection, but point to the left in the bottom half of the MFR. The force-free equilibrium of the current-carrying field of the filament requires that any net current must be directed such that its Lorentz force with the so-called strapping field (i.e., the field due to external sources passing over the filament), $J \times B_{\text {ext }}$, points downward. Therefore, the current points into the direction of the axial field in so-called sinistral filaments (as defined in Martin, 1998), which are, therefore, right-handed. The threads in sinistral filaments point to the left, which is also the prevailing direction of deviation of the filament barbs from the axial field and spine of the filament (see, e.g., Figures 10 and 11 in Martin 1998). Therefore, the direction of the threads (and barbs) in quiescent filaments demonstrates that their structure is that of an MFR. The mirror image, with the opposite handedness and opposite direction of threads and barbs, is observed for so-called dextral quiescent filaments, which, of course, equally clearly indicate an MFR field. Finally, since FCs embody high magnetic shear at the PIL, the flux complex they are embedded in generally carries a net current (Török and Kliem, 2003 Török et al, 2014b, Dalmasse et al, 2015), which is also observed (Liu et al, 2017, Kontogiannis et al, 2017).

Cavities: The low-density cavities of quiescent prominences (for a review on quiescent cavities, see, Gibson, 2015) provide strong evidence for the evolution from SMA to MFR structure via resistive processes as discussed in Section 2.2. Case studies of high-lying (i.e., old) prominences revealed elliptical cavity shapes fully detached from the limb, suggestive of an MFR viewed along the axis and incompatible with an SMA. The prominence material is located in the bottom half of the ellipse and sometimes shows concave-upward fine structure (Gibson et al, 2010, Karna et al, 2015b). A particularly clear example is shown in Figure 16. Karna et al (2015a) studied over 400 cases, focusing on the properties in the middle of the cavity's life time. The overall 3D topology of cavities could be characterized as a long tube with an elliptical cross section. Most cavities then were partial ellipses, whose midpoint was elevated above the limb, with the prominence material reaching up to about the midpoint, i.e., being situated in the lower half of an apparent full ellipse centered on the observed midpoint and covering the 
prominence material, except for its feet. This indicates the SMA-to-MFR transformation theoretically expected from the flux cancellation process, with the area of the cavity outside the virtual ellipse still being an SMA. The geometry also indicates magnetic dips at the position of the prominence material. Forland et al (2013) showed that partially elliptical cavity shapes typically evolve into full ellipses at gradually increasing height, which eventually fully detach from the limb and then develop an inverse teardrop shape. They also found that prominences in cavities at the latter stage are about seven times more likely to erupt than those in elliptical and semi-circularly shaped cavities and suggested a combination of current sheet formation at the bottom tip of the cavity and MFR instability as the cause for the increased propensity for eruption. Slow reconnection in the CS would supply the cavity/MFR with flux, such that its center rises, eventually reaching the critical height for onset of the torus instability (Kliem and Török, 2006). These findings convincingly suggest that the SMA-to-MFR evolution continues throughout the lifetime of quiescent prominences and directly leads to their eruption. We conclude that the magnetic fields carrying quiescent prominences are in a transitional SMA-to-MFR stage throughout their lifetime. Therefore, the notion that FCs carrying quiescent prominences are either SMAs or MFRs appears to be overly restrictive. Finally note, that sometimes eruptive filaments/prominences show evidence of helical structure (e.g., Vrsnak, 1990).

Spinning Motions: Using high-resolution, high-cadence coronal images obtained from STEREO/EUVI, Wang and Stenborg (2010) found that some coronal cavities appear as continuously spinning structures, with sky-plane projected flow speeds in the range of $5-10 \mathrm{~km} \mathrm{~s}^{-1}$ that often persist for several days. They argued that such persistent swirling motions provide strong evidence that the cavities are MFRs viewed along their axis. SMA hybrids with helical field lines (Fig. 13) could exhibit swirling motions in their IP/NP interface(see dips discussion in 3.1.2). In this case, however, the center of the swirling motions will be located below the prominence apex. In the MFR case, the center of the swirling motions lies above the prominence top, consistent with the existing observations. Moreover, helical field lines exist only in a shell of relatively small flux content, whereas the main central part of the flux in this hybrid structure is only sheared, not helical.

However, we should be conscious of the impact of projection effects on observational studies. Ba̧k-Stȩślicka et al (2013) using COMP observations, found concentric rings of the line-of-sight velocity within cavities; the velocity rings provide strong evidence of an MFR structure, as they suggest flows along flux surfaces of an MFR. On the other hand, Schmieder et al (2017) reconstructed a seemingly helical prominence observed by IRIS with slit-jaw images and spectra and deduced that the spiral-like structure of the prominence observed in the plane of the sky is mainly due to the projection effect of long arches of threads. In other words, the actual 3D morphology of the cavities needs to be taken into account for the proper interpretation of prominence fine structure as discussed in Gunár et al (e.g., 2018).

Polarization: Synthetic observations of the linear polarization in forbidden IR lines in the corona show little differences between cylindrical MFRs and sheared arcades (Rachmeler et al, 2013), unless observations at altitudes below the inner field of view (FOV) of COMP are available. On the other hand, Rachmeler et al (2013) showed that observations of the circular polarization can distinguish between SMAs and MFRs, since in the former case the strong 
signal is close to the limb whereas in the latter case a circular ring of enhanced signal is anticipated (see Figures 5 and 7 in Rachmeler et al, 2013).

\section{MFR Signatures in AR Filament Channels}

Thread Orientation: Measurements of the magnetic field in AR prominences are not yet possible, because these form at low heights, where scattered light from the limb is too strong. For AR filaments well within the solar disk, vector magnetograms have revealed inverse field direction across the photospheric PIL, i.e. BPs, for some FCs (Jiang et al, 2014, Yardley et al, 2016, Chandra et al, 2017; Kliem et al, 2020). Measuring the direction of threads in AR filaments requires high resolution and is still in its early stages. The data available so far tend to point to a direction opposite to that of quiescent filaments (NP instead of IP). A clear example is shown in Wang et al (2015), where the threads of a filament contained in left-handed field point to the left, a result consistent in principle with both an MFR and SMA. On the other hand, a few AR filaments have been found to show winding threads directly indicating an MFR; see an example suggestive of one field-line turn in Xue et al (2016).

Cavities: Cavities around AR filaments are often seen to form during eruptions as an element of the three-part structure of white-light CMEs (Illing and Hundhausen, 1985), but, with one exception, have not yet been observed as equilibrium structures prior to eruptions (Patsourakos et al, 2010). This is because AR filaments are very low-lying, so that their cavities are likely obscured by the AR emission structures. An AR cavity has been observed, in one case, for about one hour during the slow-rise phase prior to the onset of a fast CME. The helical fine structure of the embedded filament was suggestive of an MFR (Chen et al, 2014).

Dips: From the above observations, in particular from the structure of AR prominences, it is clear that prominence material in ARs is far less associated with magnetic dips than in the QS. This is not yet fully understood but is consistent with the following considerations. Since the nearly horizontal fields in AR filament channels have much lower altitudes, they can be supplied with cool material from below (e.g. via chromospheric jets) frequently and thus the material of each individual thread does not need to be trapped for a long time for a continuous presence of the prominence as a whole. This is in line with numerical simulations of prominence support by long field lines without dips (Karpen et al, 2001; Luna et al, 2012). Magnetic dips are much more difficult to create by the prominence material, because the Lorentz force in ARs exceeds the gravitational force much more than in the QS. Indeed, AR prominences are much more variable and shortlived than their quiescent analogs, with up and downflows being omnipresent. Consequently, their threads are more likely to populate both concave-upward and concave-downward field lines, so that an MFR structure is more difficult to infer observationally. However, ARs provide further opportunities to infer MFRs, which we discuss below.

Sigmoids: The coronal signature of many AR filament channels is a SXR sigmoid. As discussed in Section 2.2.3 single-S sigmoids are considered to be a strong observational indication of an MFR, especially when their middle section crosses the PIL in the inverse direction (Green and Kliem, 2009, Green et al, 2011) and when their ends turn around strongly to point toward the center of the sigmoid (Kliem et al, 2020). Diffuse sigmoids, on the other hand, are composed of many loops of a regular arc or $\mathrm{J}$ shape, which only collectively form an $\mathrm{S}$ shape. There- 


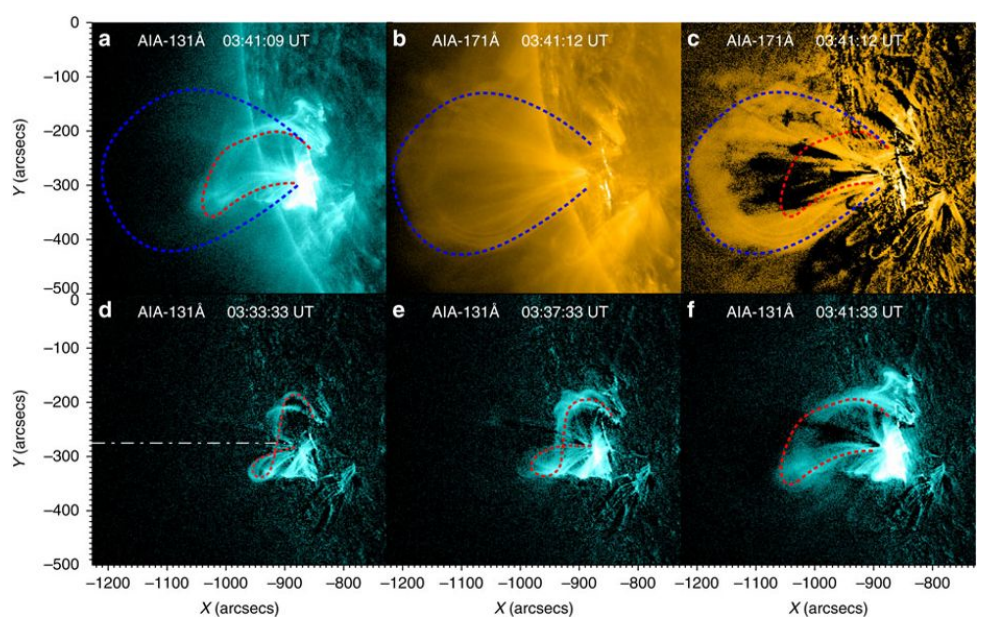

Fig. 17 Evolution of a hot channel (presumably an MFR) during the March 8, 2011 eruption. The channel (marked by the red dotted line) is visible in the hot AIA $131 \AA$ passband (a), but absent in the cool $171 \AA$ passband (b). (c) Difference image (171 $\AA$; base image at 03:20:41 UT), showing the compression front of the eruption (marked by the blue dotted line). (d)-(f) Sequence of difference images (131 ^; base image at 03:20:09 UT), showing the evolution of the hot channel (red dotted lines). The channel apparently transformed from a writhed sigmoidal shape into a semi-circular shape (adopted from Zhang et al, 2012).

fore, they are very suggestive of an SMA structure. It is very likely that the transition from the diffuse form via double Js to single $\mathrm{S}$ sigmoids, which occurs during extended periods of photospheric flux cancellation (Green and Kliem, 2009. Green et al, 2011, Savcheva et al, 2012), outlines the SMA-to-MFR transformation conjectured by van Ballegooijen and Martens (1989), Moore et al (2001). The transformation relies on persistent, ubiquitous flux cancellation events (one of which is sketched in Figure 10, where reconnection progressively increases the highly sheared and typically only moderately twisted flux of the FC. However, the twist may or may not increase in this process; compare the schematics in van Ballegooijen and Martens (1989) and Green et al (2011).

Hot channels: A new line of observational evidence for MFRs emerged with the so-called EUV "hot-channel" structures revealed by SDO/AIA observations (Zhang et al, 2012, Cheng et al, 2013, Patsourakos et al, 2013, Nindos et al, 2015. Zhou et al, 2017, Veronig et al, 2018). These structures exhibit a sigmoidal shape when seen against the disk (Liu et al, 2010) and resemble a hot blob or arc when seen above the limb (Cheng et al, 2011, 2014a).

The hot channel can be clearly seen in the AIA $131 \AA$ passband, which is sensitive to high plasma temperatures $\sim 10 \mathrm{MK}$, while it is completely absent in the AIA $193 \AA$ passband, which is sensitive to AR coronal temperatures $(\sim 2$ $\mathrm{MK}$ ), and in the AIA $171 \AA$ passband that is sensitive to quiet corona/transition region temperatures of $\sim 0.6 \mathrm{MK}$ (Figure 17).

Since these hot temperatures are higher than those of SXR sigmoids, the channels are more closely associated in time with flaring activity, which provides plasma heating through reconnection. The statistical study of Nindos et al (2015) showed that $49 \%$ of major eruptive flares (above GOES M-class) involve hot channels. 
These hot channels frequently exhibit hook-shaped ribbons during flares, an indirect indication for an MFR (Démoulin et al, 1996, Cheng and Ding, 2016, Zhao et al, 2016). Note that the hooks would extend into spirals for highly twisted MFRs (Démoulin et al, 1996). In the case of a pre-existing MFR, hook-shaped ribbons are formed right from the flare onset. MFRs possess topological features, such as HFT/QSLs and BP/BPSS (see also section 3.1.2). Current sheets are prone to form along these topological features, typically on Alfvénic timescales, when the magnetic field is evolving. As a result, flare-reconnection can start early during MFR eruptions and illuminate ribbons, both under the flare CS and the hookshaped ribbon extensions (e.g., Aulanier et al, 2010, Zuccarello et al, 2015). In the case of an SMA though, there could be some delay between flare onset and their appearance. This is because quasi-linear ribbons will appear first under the flare CS, and then their hook-shaped extensions will develop once the MFR starts to form during the eruption. Therefore, observations of hook-shaped ribbons have a limited potential to distinguish between SMAs and MFRs, unless possibly when observing them during confined flares preceding an eruption.

Unlike filaments, which often become fragmented during the eruption, the EUV hot-channel structures maintain their coherence throughout the eruption process. Unlike SXR sigmoids, which quickly fade away into the background (thus cannot be traced for long), the channels can often be continuously traced until they leave the AIA FOV.

Most of the identifications and studies of hot channels have focused on occurrences in the course of eruptions. Here, we are interested in evidence for preexisting MFRs, meaning MFRs that formed clearly before the eruptions, so we focus on such evidence below. In events that feature a hot channel already in the slow-rise phase, the channel displays a morphology similar to its appearance after the main eruption onset (Zhang et al, 2012, Cheng et al, 2013). Patsourakos et al (2013) described a case where an MFR was formed long before an eruption. They identified a hot structure that formed during a confined flare and erupted seven hours later. This hot structure had an oval (blob-like) shape and was formed above a cusp straddling the corresponding post-flare loop arcade. The cusp then corresponded to the CS associated with the ascent of the magnetic flux which gave rise to the corresponding confined flare. MFR formation (or enhancement) during eruptive flares, is a common element of MHD models, whether they do (e.g., van Ballegooijen and Martens, 1989) or do not include (e.g., Karpen et al, 2012) a preeruption flux rope. Another important take-away from Patsourakos et al (2013) was that, by limiting the observational window only around the eruptive flare, one would have missed the confined flare event and hence would have been led to different conclusions about the formation time of the erupting MFR. Chintzoglou et al (2015), Kumar et al (2017), James et al (2018), Liu et al (2018), and Kliem et al (2020) essentially reached the same conclusion for other events: MFRs were formed during confined flares. NLFFF extrapolations revealed twisted field lines in the places where hot structures were formed during some of the reported confined flares.

Our review of the SMA and MFR signatures can be summarized as follows. MFRs in FCs are expected to form through a transition from an SMA, often driven by flux cancellation. Since flux cancellation operates gradually and intermittently due to the fragmented structure of the photospheric magnetic flux, FCs should be in a transition state for a long time. Moreover, as long as flux cancellation 


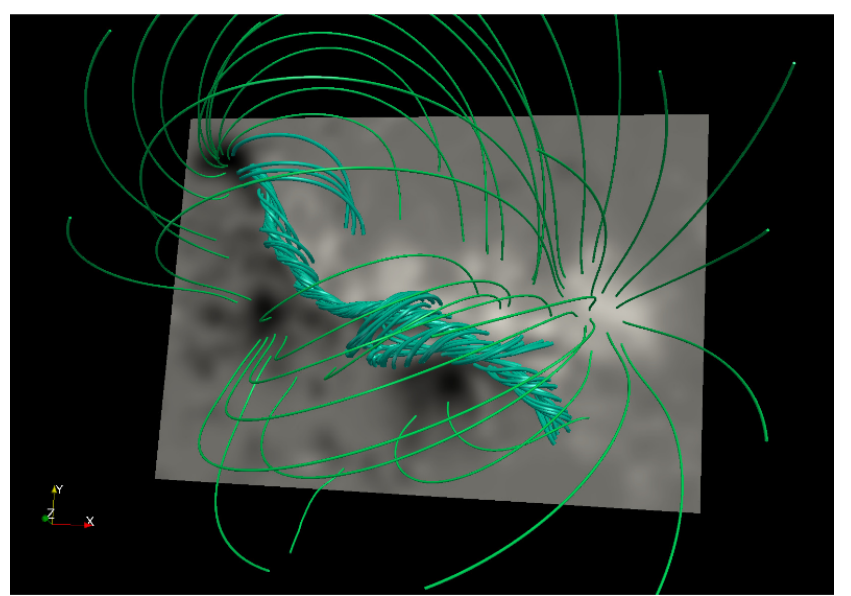

Fig. 18 NLFFF extrapolation of the magnetic field of a filament channel, showing an incoherent MFR structure (two MFRs separated by a short SMA section) shortly before its two relatively coherent MFR sections successively erupted to cause two major CMEs (from Chintzoglou et al 2015). (C)AAS. Reproduced with permission.

episodes still occur, an already existing MFR will be enhanced by additional flux. The additional flux does not necessarily extend along the whole FC, especially not for long ones, and will branch off from the main body of the MFR in an arcadelike manner toward its photospheric roots, which may be seen as barbs. Therefore, although the observations cited in Section 3.2 .2 clearly indicate that (at least) quiescent prominences form when the transition to an MFR structure has strongly advanced, the MFR may still be completing its transition or enhancement. One should not expect an MFR that is perfectly coherent from end to end. During the transition, one may find a mix of arcade-like and rope-like sections(both (typically in a small number) with a growing degree of coherence. These may take the form of flux branching off from an otherwise fully developed MFR (Guo et al, 2013) or of a section of SMA structure adjacent to an MFR (Guo et al, 2010) or between two MFRs (Chintzoglou et al, 2015, see Figure 18) or coexisting sheared and twisted field lines of different extension (Figure 3(e)). It is an open question how coherent an MFR has to be (in terms of flux connecting its two main footpoints) to allow the initiation of a CME by a loss of equilibrium or, equivalently, an ideal MHD instability. In addition to this gradual process of MFR formation, the observations of EUV hot channels indicate that MFRs can be formed or extended by reconnection on a much shorter time scale during periods of low-level flaring activity which precede a subsequent major eruption into a CME. These can be a confined flare or the slow-rise phase leading to the major eruption (e.g., Liu et al, 2010, Zhang et al, 2012; Patsourakos et al, 2013).

\subsection{Differences in eruption signatures between SMA and MFR}

Although our focus is on the pre-eruptive magnetic field configuration, we assess briefly below some potential SMA/MFR differences during the eruption phase as these are often used in the literature. 
Flare Timing: A sufficiently sheared SMA will collapse to form a vertical CS extending upward from the PIL. Reconnection in the CS tends to set in immediately, which marks the onset of a flare, and forms an MFR at the tip of the CS (e.g. Mikic and Linker, 1994; Karpen et al, 2012). Depending on parameters, the MFR can start to rise immediately or could be delayed. Hence, in an erupting SMA, a flare will start prior to or simultaneously with the onset of an eruption. In comparison, the timing between flare and CME onset is reversed in an MFR if the MFR is of BP topology. In this case, a vertical CS forms only when the unstable upper part of the MFR has risen sufficiently (Gibson and Fan, 2006b). However, if an X-type HFT exists at the underside of an unstable MFR, then the HFT collapses into a vertical CS, and reconnection commences, simultaneously with the onset of the rise, i.e., flare and CME commence simultaneously. However, since flare onset and CME onset are rather well synchronized in the majority of eruptions (Zhang and Dere, 2006, Temmer et al, 2010, Cheng et al, 2020), with a trend though that CME rise starts before the impulsive flare energy release (in terms of the flare SXR and HXR emission, respectively) as shown in Bein et al (2012); Berkebile-Stoiser et al (2012), the time difference between them can only rarely be used to discriminate between SMA and MFR as the pre-eruptive structure.

Partial Eruptions: It is frequently observed that the top part of a filament channel rises and erupts explosively, while the bottom part remains on the Sun, apparently undisturbed (e.g., Pevtsov, 2002). Partial eruptions from an SMA configuration seem straightforward to understand as flare reconnection and CS formation can occur anywhere along such configurations transforming only part of the SMA into the escaping MFR (e.g., Cheng et al, 2014b). H $\alpha$ observations sometimes show flare ribbons appear and spread far from the observed dark filament, implying that the eruption and reconnection involve only the FC magnetic flux above the flux carrying the cool material. However, MHD simulations have shown that a single MFR can split via internal reconnection in the early phase of its eruption (e.g., Gibson and Fan, 2006b; Kliem et al, 2014b), as previously suggested by Gilbert et al (2001). Such a split will occur when the main part of the MFR, including its axis, satisfies the criterion for an instability while the bottom part of the MFR is tied to the photosphere in a BP. Other works have invoked the existence of a so-called "double-decker" configuration of two MFRs lying on top of each other prior to an eruption and assuming that only the upper one, which may not carry filament material, erupts (e.g., Liu et al, 2012, Cheng et al, 2014b; Kliem et al, 2014b, Dhakal et al, 2018). It seems, therefore, that while the vertical splitting of the FC flux follows naturally for SMA topology, it requires special conditions to explain with an MFR topology.

Ideal Instabilities: The internal current distributions differ strongly in SMAs and MFRs, which is relevant for the development of ideal instabilities that may initiate an eruption. SMAs and MFRs are both force-free, so they contain fieldaligned currents whose paths follow exactly the associated stressed field lines. Just like the field-line shapes and connectivity, current paths are very different in SMAs and MFRs. In particular, the currents in an MFR go all the way from one end of the rope to the other. This is shown, e.g, in Figures 15 and 19. In an SMA, on the other hand, magnetic shear decreases with distance from the PIL. Therefore, electric currents can connect areas of very large and very small shear, as can be seen by looking at the field lines in, e.g., Fig.13. 
Such a coronal distribution of electric currents is unfavorable for the occurrence of ideal loss-of-equilibrium scenarios and/or the torus instability. This is because, neutralized currents shield the main currents from which Laplace forces are calculated, so there can be no repelling between distant-components and/or subphotospheric-images of the modeled currents "wires". Along the same lines, the differential shear by itself cannot produce local twists of more than about half a turn in an SMA. Therefore, SMAs are not prone to the helical kink instability, either. It follows that an isolated bipolar SMA is an ideally stable configuration, so that it cannot erupt. This was first demonstrated by Roumeliotis et al (1994), Mikic and Linker (1994), and Amari et al (1996). The only way to make an SMA erupt is to provide an external perturbation, such as, e.g., in the magnetic breakout model, and to produce a CS in which flare reconnection can occur. In other words, the detection and quantification of an ideal instability as the driver of an eruption appears to be the clearest discriminator between an SMA and an MFR as the eruption origin. Unfortunately, the potential of ideal instabilities is (yet) to be fully investigated, mostly due to observational constraints. For example, it is difficult to quantify the role of the torus instability because incomplete coverage of the photospheric magnetic field and lack of high-cadence multi-viewpoint EUV imaging complicates the inference of the height of the eruption onset and the magnetic field decay index at that height (see detail, e.g., in Cheng et al, 2020).

Additionally, in highly energetic cases, an SMA could transform into an MFR too rapidly for our observational cadence to provide evidence for an instability prior to the inevitable onset of reconnection.

\section{Summary}

The goal of our ISSI team was to investigate and to resolve, if possible, the debate on whether the pre-eruptive magnetic configurations of CMEs are either SMAs or MFRs. Our objectives were to: (1) examine the nature of pre-eruptive configurations of CMEs as revealed by current observations, numerical modeling, and theoretical inferences, (2) identify the origins (theoretical and observational) of the debate and then (3) attempt to reconcile the differences and sketch a path forward on this issue.

To this end, we reviewed the current state of knowledge on the formation mechanisms and magnetic configurations of the pre-eruptive structure of CMEs (Sec. 2 ). We then reviewed the theoretical expectations and observational signatures that are commonly used to interpret observed features as SMAs or MFRs (Sec. 3). In Table 1, we summarize those signatures and their association with either an SMA or an MFR. It is obvious that several signatures can be attributed to both types of pre-eruptive configurations. The main reasons for the dearth of unique signatures are, on one hand, the inherent ambiguities in imaging and photospheric magnetic field observations, which form the bulk of the observables, (e.g., line-of-sight integration, signal-to-noise ratios, spatial resolution, lack of multi-height magnetic field measurements, non-force free photospheric magnetic field, etc.) and, on the other hand the fact that SMAs and MFRs in FCs often exist in states with partly similar properties (e.g., nearly straight field lines in the center of both highly sheared SMAs and highly sheared but weakly twisted MFRs); namely, the co-exist in a hybrid state. 
Table 1 Observational signatures of pre-eruptive SMAs and MFRs. The term SMA refers primarily to the 3D strongly sheared S-shaped SMAs discussed in Sec. 3.1 IP and NP refer to inverse and normal prominence polarity (Sec. 3.1 and 3.2. BP and HFT correspond to bald patches and hyperbolic flux tubes (Sec. 3.1.2). In the case of SMA-MFR hybrids the listed signatures refer to the flux that embodies the SMA and MFR components of the hybrid structure.

\begin{tabular}{|c|c|c|c|}
\hline Observable & SMA & MFR & Remarks \\
\hline $\begin{array}{l}\text { single-S sigmoid } \\
\begin{array}{l}2.2 .3 \\
2.2 .2\end{array}\end{array}$ & $\mathrm{~N}$ & $\mathrm{Y}$ & \\
\hline $\begin{array}{l}\text { diffuse } \text { sigmoid } \\
\begin{array}{ll}2.2 .2 & 3.2 .2\end{array}\end{array}$ & $\mathrm{Y}$ & $\mathrm{N}$ & \\
\hline prominence dips & $\mathrm{Y}$ & $\mathrm{Y}$ & $\begin{array}{l}\text { SMA: IP and NP dips in prominence main body } \\
\text { and top/periphery respectively for strong shear and } \\
\text { large departures from 2.5D (no dips for arched- } \\
\text { shaped SMAs); MFR: IP dips (small-isolated } \\
\text { patches of NP dips possible when PIL surrounded } \\
\text { with parasitic polarities) }\end{array}$ \\
\hline $\begin{array}{l}\text { linear threads } \\
\text { in prominences } \\
\text { (3.1.2 }\end{array}$ & $\mathrm{Y}$ & $\mathrm{Y}$ & \\
\hline $\begin{array}{l}\text { elliptic } \quad \text { or } \\
\text { teardrop-shaped } \\
\text { EUV } \quad \text { cavities } \\
(3.2 .2\end{array}$ & $\mathrm{N}$ & $\mathrm{Y}$ & \\
\hline $\begin{array}{l}\text { spinning motions } \\
\text { in EUV cavities }\end{array}$ & $\mathrm{N}$ & $\mathrm{Y}$ & $\begin{array}{l}\text { SMA: possible but only below prominence top (not } \\
\text { observed) }\end{array}$ \\
\hline $\begin{array}{l}\text { circular polar- } \\
\text { ization in the } \\
\text { corona }(3.2 .2\end{array}$ & $\mathrm{Y}$ & $\mathrm{Y}$ & $\begin{array}{l}\text { SMA: stronger near limb; MFR: circular ring above } \\
\text { limb }\end{array}$ \\
\hline $\mathrm{BP} \sqrt{3.1 .2}$ & $\mathrm{~N}$ & $\mathrm{Y}$ & \\
\hline $\mathrm{HFT}^{3.1 .2}$ & $\mathrm{~N}$ & $\mathrm{Y}$ & \\
\hline $\begin{array}{l}\text { flare-related } \\
\text { EUV hot chan- } \\
\text { nel }(3.2 .2\end{array}$ & $\mathrm{N}$ & $\mathrm{Y}$ & $\begin{array}{l}\text { pertinent observations during confined flares prior } \\
\text { to the eruption are better suited to probe the pre- } \\
\text { eruptive configuration }\end{array}$ \\
\hline $\begin{array}{l}\text { hook-shaped rib- } \\
\text { bons } 3.2 .2\end{array}$ & $\mathrm{Y}$ & $\mathrm{Y}$ & $\begin{array}{l}\text { observed from flare onset for an MFR and possibly } \\
\text { delayed for an SMA; pertinent observations during } \\
\text { confined flares before the eruption are better suited } \\
\text { to probe the pre-eruptive configuration }\end{array}$ \\
\hline $\begin{array}{l}\text { coronal currents } \\
\text { (mapping only } \\
\text { differential shear } \\
\text { across the PIL) } \\
\\
\text { (3.3 }\end{array}$ & $\mathrm{Y}$ & $\mathrm{N}$ & \\
\hline $\begin{array}{l}\text { coronal currents } \\
\text { (end-to-end } \\
\text { along the PIL) } \\
\text { (3.3 }\end{array}$ & $\mathrm{N}$ & $\mathrm{Y}$ & \\
\hline $\begin{array}{l}\text { CME accelera- } \\
\text { tion at Alfvénic } \\
\text { speeds } 3.3\end{array}$ & $\mathrm{Y}$ & $\mathrm{Y}$ & $\begin{array}{l}\text { SMA: only at flare impulsive phase onset; MFR: } \\
\text { possible before flare impulsive phase onset. }\end{array}$ \\
\hline
\end{tabular}

We emphasize that the proper assessment of the pre-eruptive configuration of CMEs requires that the observables of Table 1 are applied to "truly" pre-eruptive states that exist up to the onset point defined in Section 1, i.e., before the speed of the ejected structure exceeds $100 \mathrm{~km} / \mathrm{s}$. It is prudent to search for these signatures 
from a few hours prior to the eruption up to the slow rise phase that often precedes CMEs (e.g., Zhang et al, 2004, Zhang and Dere, 2006). A given signature could be visible only during particular times/conditions (e.g., the 'hot channels' observed in the AIA $131 \AA$ passband discussed in Section 3.2 , and therefore, it is recommended to search for potential signatures at multiple instances during the pre-eruptive phase.

Finally, we were able to identify and reach consensus on several key issues that have fuelled the debate in the past:

- Observations and data-constrained modeling indicate that highly twisted preeruptive MFRs $(N>2)$ are exceedingly rare.

- The observational evidence for bodily emerging MFRs is scarce, which is in agreement with almost all flux-emergence simulations.

- Different physical mechanisms (flux emergence/cancellation, helicity condensation, tether-cutting reconnection) can be involved in the formation of SMAs and MFRs, and their respective contributions depend on the evolutionary stage and location (AR, between ARs, QS) of the pre-eruptive configuration.

- The amount of magnetic flux associated with SMAs and MFRs is hard to pin down either with modeling or observations. For example, magnetic cancellation studies suggest that a significant amount of the total AR flux can be available for inclusion into an MFR, while NLFFF modeling often suggests that MFRs contain only a small fraction of the AR flux. This is an area that requires attention (see next section).

- In many cases, pre-eruptive configurations seem to undergo a slow transition from SMA to MFR during their evolution. This transition likely accelerates during the slow rise phase prior to an eruption.

- The spatial coherence of the pre-eruptive magnetic configuration could be used to determine the stage of this transition, i.e., whether the configuration is closer to an SMA or an MFR state, and whether the configuration is 'ready to erupt'. Methods to quantify the coherence, using modeling or observations, should be pursued.

The most important conclusion from our deliberations is that the debate on SMA versus MFR may be a 'red herring". Neither the observations nor the theory nor numerical modeling support a fixed interpretation of the pre-eruptive configuration as being either an SMA or an MFR. The most sensible conclusion is therefore, that the pre-eruptive state is a 'hybrid' configuration that contains both sheared and twisted field lines at varying proportions. Moreover, one should not expect that the configuration is perfectly coherent from end to end. In most cases, we are observing a magnetic system in transition from SMA to MFR. Depending on the state of this evolution, the configuration may be more "SMA-like" or more "MFR-like".

Therefore, the question now shifts to determining the degree of 'SMA-ness' or equivalently of 'MFR-ness' of the magnetic configuration of a given FC. Quantifying this degree may be performed by calculating the (dimensional) ratio $\mathcal{R}_{\Phi_{B}}$ between the sheared and twisted flux (see also discussion of Section 1.1 on hybrid structures). $\mathcal{R}_{\Phi_{B}}$ is a parameter that may be derived from magnetic field cubes resulting from either MHD simulations or from NLFF extrapolations from magnetograph data. Another pertinent metric may be based on the decomposition of magnetic helicity in self and mutual terms (see section 2.3. Clearly, new concepts 
and tools are required to address 'SMA-ness' and 'MFR-ness'. Successful investigation of this question should lead to a better understanding of CME initiation and could eventually improve our ability to predict their occurrence by inferring key eruptivity parameters pertinent to ideal MHD instabilities (e.g., twist, decay index of the overlying field) and magnetic reconnection (e.g., plasma heating and particle acceleration, reconnected flux). In the next section, we propose a path forward to make progress on the outstanding issues in the theory, observations, and modeling of pre-eruption FC magnetic structure.

\section{Path Forward}

The final task of our ISSI team was to recommend a path forward for the difficult problems described in the previous sections. We compiled a list of recommendations for further studies; one set deals with observations and data analysis issues (Section 5.1) and the other deals with modeling and simulations issues(Section 5.2 . The two sets list several of the major issues that came up in our deliberations but they are by no means complete. In addition, we present, in Appendix A. a possible approach for addressing some of these problems and discuss it in the context of upcoming and future observational capabilities.

\subsection{Suggested Path Forward for Observations and Data Analysis}

Perform systematic observations of FC formation. In our deliberations, we realized that there exist only a handful of observations of FC formation, based mostly on the orientation of chromospheric fibrils (e.g., Gaizauskas et al, 1997; Wang and Muglach, 2007). They concern relatively small FCs at the periphery of ARs, that form within 1-2 days. We could not find any study of the full timeline of formation of large quiescent FCs. The reasons may be that these FC are quite extended, form over long time-periods (weeks), and the existing high-resolution $\mathrm{H} \alpha$ capabilities (needed to see fibril orientation) cannot cover the required spatial extensions and time periods. In principle, fibrils could be observed also in the EUV, but again one would need high resolution (IRIS), large FOV, and sufficiently long observation periods. Vector data could help to observe the development of shear, but the problem there is that most large FCs form in weak-field regions, where the field strengths are below the HMI sensitivity. Synoptic magnetic field and chromospheric observations from various viewpoints, i.e, terrestrial from SOT, HMI and DKIST and off the Sun-Earth line and above the ecliptic by Solar Orbiter's PHI, SPICE and EUI may allow the tracking the evolutionary paths of FC over longer time periods.

Determine the role of cancellation in creating the pre-eruptive configuration. Until now magnetic flux cancellation has been almost exclusively observed in regions where flux emergence has largely ceased. Flux cancellation could also occur in areas exhibiting ongoing flux emergence, something that needs investigation, with the first steps towards this direction taken by Chintzoglou et al (2019). In addition, the estimation of the magnetic flux associated with flux cancellation (Section 2.2.3) and with coronal reconnection estimated during confined flaring events (e.g., Veronig and Polanec, 2015, Tschernitz et al, 2018) should clarify the 
respective role of flux cancellation, which is a continual and small-scale process, and coronal reconnection, which is a transient process with consequences for the large-scale structure in establishing a pre-eruptive magnetic configuration.

Perform statistical surveys for bodily emerging MFRs. The observational cases in support of FC formation via bodily/rigid MFR emergence are scarce. Moreover, the proposed interpretations are merely suggestive and inconclusive. To make progress, we need a comprehensive analysis of several cases. It is important to strictly consider FCs forming in isolation, as in Lites et al (2010), to strengthen the interpretations. Since flux emergence and FC formation may take more than a day, long-duration observing sequences will be necessary. Large coverage of the solar surface will make this exercise much easier, since the emergence may occur anywhere on the Sun and knowledge of the time history of the region is needed to decide whether the emergence occurs in a 'filament-free' location.

Determine the role of small-scale convective flows on the injection of helicity into the corona. It is widely believed that convective flows at the photosphere are responsible for injecting the free energy that powers coronal heating, but their role in helicity injection has yet to be determined accurately. Vortical flows in inter-granular lanes are commonly observed, and the random nature of the convective flows may well impart a net twist to the coronal field, eventually leading to the formation of FCs via helicity condensation, but this needs to be determined quantitatively. Observational studies are required that measure accurately the amount of helicity injected into the corona by the granular and supergranular motions.

Apply the SMA/MFR diagnostics matrix (Table1) to a statistically meaningful number of cases. The maximum possible number of the observables of Table 1 should be considered. Such studies should provide a more coherent picture of the SMA/MFR signatures. Attention should be paid to 'hybrid' configurations, as they may be the norm. In particular, it should be examined how these structures evolve in time, and what is their typical state at eruption onset. In all cases, the properties (twist, helicity, magnetic flux, etc.) of each component should be quantified.

Benchmark the pre-eruptive magnetic configurations resulting from different NLFFF techniques. The properties of modeled MFRs seem to depend on the particular NLFFF magnetic field extrapolations method, e.g based on the widely-used Wiegelmann et al (2014) code, or flux-rope insertion techniques (van Ballegooijen, 2004), with the former typically leading to thinner MFRs (Liu et al, 2016). These effects should be investigated in more detail, and should be compared also with results from magneto-frictional techniques (e.g., Mackay et al, 2011, Fisher et al, 2015), non-force-free field extrapolations ( $\mathrm{Hu}$ and Dasgupta, 2008, Zhu et al, 2016), and MHD simulations (e.g., Török et al, 2018a).

Perform systematic studies of magnetic field in both quiescent and AR filaments. The work on the IP and/or NP magnetic configurations stems from observational studies in the 1980s. A modern era study is needed to provide more complete and reliable observations.

Determine whether all sigmoids are signatures of MFRs. Study more cases of sigmoid morphology and evolution in combination with coronal NLFFF and magnetofrictional modeling (as in Savcheva and van Ballegooijen, 2009, Savcheva et al, 2012, Zhao et al, 2016, Yardley et al, 2018b), to clarify whether diffuse sigmoids already harbor an MFR and at what evolutionary stage. 
Determine the decay index of the coronal field at eruption onset. Estimates of the decay index at the point of eruption onset for many events can help deciding between MHD instability models, which require an MFR to exist, and reconnection models, which operate in an SMA. A significant correlation between the estimated decay index at the observed onset height of eruptions with the theoretically expected critical decay index for the onset of the torus instability found for a large sample of events supports the ideal MHD model, i.e., the pre-existence of an MFR. The reliability of present estimates (e.g., Cheng et al, 2020) will be considerably improved when the precise tracking of eruptions near the limb with SDO/AIA data can be combined with simultaneous magnetograms of the source region from the Solar Orbiter in quadrature or from an L4 or L5 mission.

\subsection{Suggested Path Forward for Modeling and Simulations}

Compare systematically the pre-eruptive configurations resulting from simulations of different FC formation mechanisms. It is currently not possible to use the same boundary condition (same magnetogram) to inter-compare flux emergence, flux cancellation and helicity condensation simulations because they inherently change the photospheric boundary conditions as the models evolve. A systematic approach for this purpose is needed, but has not yet been developed. However, even exploiting existing simulations to compare the evolution and properties of pre-eruptive configurations produced by different mechanisms will lead to useful insights, as long as it is done in a systematic fashion.

Perform comparative studies of full and partial flux-tube emergence. A comparative study between the full (bodily) and partial emergence seen in fluxemergence simulations is sorely needed, aiming specifically to identify photospheric observational signatures that would allow one to distinguish between the two cases. If such signatures exist, bodily-emerging MFRs could be identified, allowing their occurrence rate to be quantified. Such information would also provide valuable insight on the properties of flux tubes in the solar interior. Another important issue is to determine under which conditions a bodily-emerged MFR can erupt. Furthermore, the differences between the properties of bodily-emerged and postemergence MFRs, formed by reconnection, have not yet been studied.

Origins of photospheric shear. Photospheric motions, such as shearing and rotation, which are driven by the Lorentz force when e.g. emerging twisted field expands into the corona, have long been studied for their role in the formation of MFRs and SMAs. In addition, the diverging motion of the polarities, which follows the emergence of the flux tube can act as a source of shearing at the photosphere. We must systematically quantify the flows and investigate their physical origins. Improve realism of flux-emergence simulations. More realistic models of the flux-emergence process are crucial for the study of pre-eruptive structures. They are necessary in order to understand the role of flux cancellation in the formation of pre-eruptive structures and its coupling with magnetoconvection. The proper treatment of the physics of the lower solar atmosphere, where current models predict that MFRs initially form, are crucial to assess both the topology of the pre-eruptive strucures and also their mass and energy contents. In addition, the realistic treatment of the energy equation and the correct treatment of ion 
population are needed to accurately forward model the numerical simulations and compare with observations.

Determine the FC magnetic structure that results from helicity condensation. The amount of twist that can accumulate in an FC determines to a large degree its structural properties. Simulations of helicity condensation, to date, invariably resulted in an SMA configuration, but these studies were not performed at the observed scales for coronal helicity injection. It is unclear whether this result will hold in more rigorous calculations, or some type of MFR will be formed instead, and whether the helicity condensation process can account for the observed properties of FCs (e.g., their size and amount of shear/twist).

Determine degree of MFR coherence to allow onset of eruption. Perform data-constrained and data-driven numerical modeling of eruptions using MFRs with different degrees of coherence as initial condition. Such MFRs have been found in extrapolated NLFFFs and can also be constructed using the flux-rope insertion method or analytic MFR models (e.g., Titov et al, 2014, 2018). Initial conditions obtained with the novel non-force free extrapolation methods should also be considered.

Acknowledgements We acknowledge the International Space Science Institute (ISSI) in Bern Switzerland for their generous support for travel and accommodations. The authors would like to thank the two anonymous referees for their very useful comments and suggestions on the manuscript. A.V. was supported by NASA grant NNX16AH70G and the LWS program through NNX15AT42G under ROSES NNH13ZDA001N. B.K. acknowledges useful discussions with Antonia Savcheva and Nishu Karna and support by the DFG and by NASA under Grants 80NSSC17K0016, 80NSSC18K1705, 80NSSC19K0082, and 80NSSC19K0860. J.Z. is supported by NASA grant NNH17ZDA001N-HSWO2R. J.E.L. acknowledges support by the NASA Living With a Star \& Solar and Heliospheric Physics programs, and the Office of Naval Research 6.1 Program and by the NRL-Hinode analysis Program; the simulations were performed under a grant of computer time from the DoD HPC program. S.L.Y. would like to acknowledge STFC for support via the Consolidated Grant SMC1/YST025 and for a PhD Studentship. T.T. was supported by NASA's HGI and HSR programs (awards 80NSSC19K0263 and 80NSSC19K0858) and by NSF's PREEVENTS and Solar Terrestrial programs (awards ICER-1854790 and AGS-1923365). X.C. is funded by NSFC grants 11722325 , 11733003, 11790303, Jiangsu NSF grants BK20170011, and "Dengfeng B" program of Nanjing University. V.A. and P.S. acknowledge support by the ERC synergy grant "The Whole Sun". S.P. and A.V. would like to thank L. Vlahos for suggesting to establish an ISSI team.

\section{Appendices}

\section{A A Suggested Approach Towards Deciphering the Pre-Eruption Configuration}

We suggest that vector magnetic field measurements at multiple heights spanning from the photosphere to the lower corona and multi-viewpoint hot plasma observations in the corona could advance our understanding of pre-eruptive configurations and may eventually lead to predicting their eruptions. To demonstrate the potential of such observations, we create and analyze synthetic data from an MHD flux-emergence simulation, as follows.

First, we extract data-cubes of the vector magnetic field in the lower solar atmosphere from the MHD flux-emergence simulation of Syntelis et al (2017) (SAT17, hereafter). Note that the SAT17 simulation had a different objective. It was not meant to simulate a large-scale CME but it has all the necessary characteristics to demonstrate our approach. We select representative pre-eruptive configurations from this simulation at two consecutive times (Fig. 19). The configuration at the earlier time (top panels) consists predominantly of sheared, high-arching 


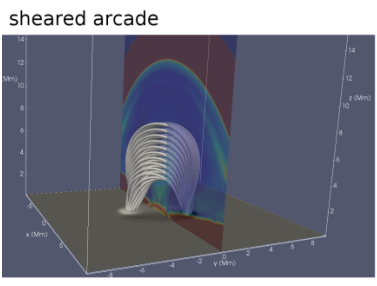

flux rope

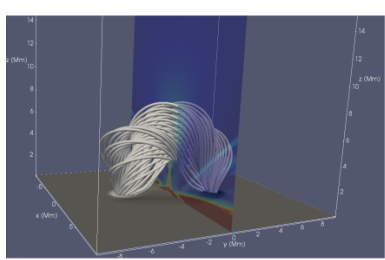

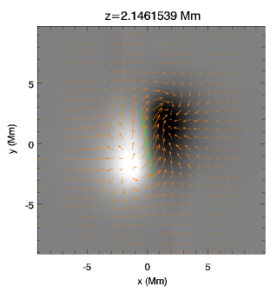

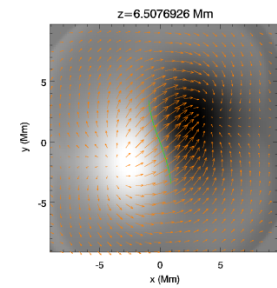

$z=2.1461539 \mathrm{Mm}$
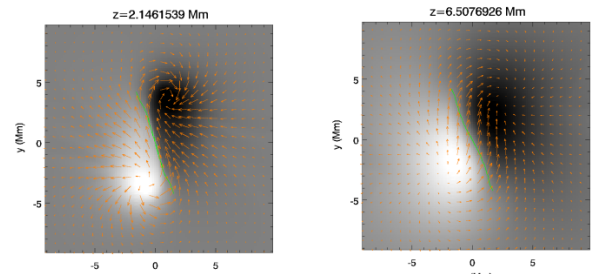

Fig. 19 Two stages of a flux-emergence simulation (Syntelis et al 2017), showing a simple SMA (top) that transitions over time into an MFR (bottom). Left: representative magnetic field lines traced from identical seed points; $B_{z}$ is shown in greyscale at the photospheric level $(z=0)$; the transparent vertical plane shows $|\mathbf{J}| /|\mathbf{B}|$. The middle and right columns show the PIL (green line) and $B_{h}$ (orange vectors) superposed on $B_{z}$ at $\mathrm{z}=2.14 \mathrm{Mm}$ and $\mathrm{z}=6.5 \mathrm{Mm}$, respectively.

field lines of similar orientation, resembling a '2.5D-like' SMA that has a much simpler structure than the flat 3D SMAs described in Section 3.1 At the later time (bottom panels), the configuration contains also twisted field lines, i.e., it has transitioned into an MFR. The central and right panels show the horizontal magnetic field, $B_{h}$, at two different heights (corresponding roughly to the lower chromosphere and middle transition region). The orientation of $B_{h}$ with respect to the PIL is significantly different for the two magnetic configurations. For the SMA, $B_{h}$ points roughly along the same direction in both atmospheric layers. For the MFR, $\mathrm{B}_{h}$ is much more sheared and flips orientation across the PIL with increasing height, which suggests a transition across the center of an MFR. This suggests that mapping the orientation of $\mathrm{B}_{h}$ as a function of height in the low solar atmosphere may be a powerful approach for assessing the evolutionary stage (SMA-to-MFR transition) of pre-eruptive configurations. The height of this transition will depend on the physical parameters of a given region on the Sun. For this reason, multiple height coverage extending to coronal heights will be needed to properly capture the evolution of the system across the range of ARs and phases of solar activity. As a side benefit, the use of constraints from multiple atmospheric layers will greatly improve the quality of NLFFF (or non-force free) extrapolations and should lead to a more accurate specification of the magnetic field in the corona.

Magnetic field observations above the photosphere should also capture field changes induced by eruptions. Such changes have been notoriously difficult to detect in photospheric magnetograms (Sudol and Harvey, 2005), leading to uncertainties of the amount of magnetic flux removed by CMEs. We demonstrate this by exploiting the recurrent eruption characteristic of the SAT17 simulation in Figure 20 We see that while the photospheric magnetic flux exhibits a smooth behavior characterized by a sharp increase followed by a plateau (black line), the magnetic flux higher up undergoes several dips, each associated with an eruption (colored lines). It is, therefore, conceivable, that we could measure the flux (and by extension, the magnetic energy) that participates in an eruption by following the magnetic flux evolution above the photosphere. Constraining the energetics of eruptions with such direct measurements would greatly advance our understanding of explosive energy release and help establish the 
geo-effective potential of CMEs at a very early stage of the eruption process (Vourlidas et al, 2019).

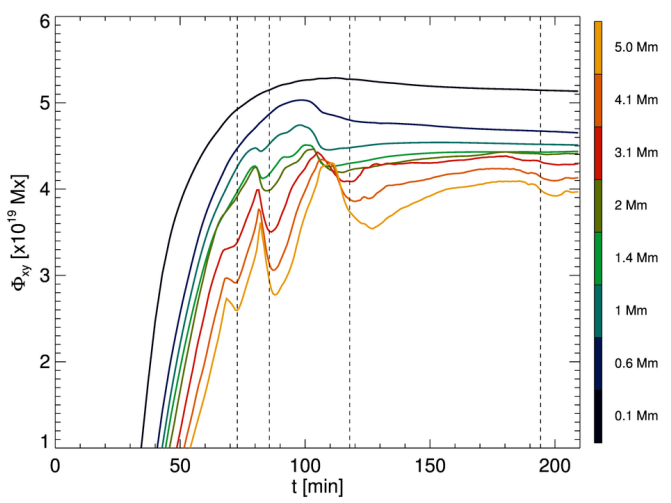

Fig. 20 Temporal evolution of magnetic flux at various heights for the Syntelis et al (2017) simulation. The snapshots employed in Figures 19 and 21 are taken before $t=50 \mathrm{~min}$. The vertical dashed lines show the kinetic energy maxima inside the numerical domain indicating the four eruptions in the simulation.

Finally, we calculate synthetic images resulting from LOS-integration of the squared electric current density, $J^{2}$, (Figure 21). These images can be considered as proxies for hightemperature plasma emissions in the EUV or SXR. We use the same simulation snapshots as in Figure 19 The top view of the SMA (Fig. 21. top left) is reminiscent of a sigmoidal structure. This is, however, easily dismissed by inspecting the side view (top right). On the other hand, both views of the MFR snapshot display a clear sigmoidal structure. We emphasize that this is just a proof-of-concept attempt. It should be expanded with more rigorous calculations by, for instance, calculating synthetic Stokes profiles from the MHD simulations and inverting them as done with real data.

These diagnostics can be exploited once vector magnetic field observations at several layers above the photosphere become available. Photospheric and chromospheric vector magnetic field observations (albeit over a limited FOV) will be available from DKIST, beginning in late 2020. In addition, the Cryogenic Near Infra-Red Spectro-Polarimeter (Cryo-NIRSP) of DKIST will obtain off-limb observations in HeI $10830 \AA$ and FeXII $10747 \AA$ for estimates of magnetic field magnitudes and orientation in prominences and coronal loops. Even though these are not vector magnetograms, the polarization information along with forward modeling can be used to decipher the nature of pre-eruptive structures within coronal cavities as shown by Rachmeler et al (2013). In late 2021, the remote sensing instruments on Solar Orbiter (Müller et al, 2020 Zouganelis et al, 2020) will start science operations, including photospheric vector magnetic field measurements by the Polarimetric and Helioseismic Imager (PHI) from non-Earth viewpoints, thus providing additional constraints on the photospheric boundary conditions for magnetic-field extrapolations and possibly allowing concurrent coronal and photospheric magnetic field estimates when combined with Cryo-NIRSP.

Multi-viewpoint observations of hot plasmas are less certain in the near future, although some research can be undertaken either with past (2007-2014) EUVI $284 \AA$ observations from STEREO-A and -B or now with comparisons between STEREO/EUVI $284 \AA$ (currently at L5 and approaching Earth) and SUVI $284 \AA$ observations. In addition, the off-ecliptic viewpoints from Solar Orbiter will supply complementary high-resolution views of prominences and hot plasmas from the EUI and SPICE instruments, which could be compared with Earth-based observations (e.g., SDO/AIA and SUVI).

Further in the future, we are looking forward to exciting mission and instrument concepts. The proposed COSMO observatory (Tomczyk et al 2016) will derive some coronal magnetic field quantities (e.g strength, azimuth) and plasma properties further from the limb than DKIST. The SOLAR-C mission, recently approved by JAXA, will carry a next-generation up- 
per atmospheric imaging spectrograph that will greatly enhance our diagnostics of the thermal structure and the dynamics of pre-eruptive configurations. Based on our earlier discussion, an EUV channel similar to the $131 \AA$ channel of SDO/AIA, at an off Sun-Earth viewpoint, along with a vector magnetograph would make great addition to a scientific payload for an L5 mission (Vourlidas 2015). An observatory at that location could play a major role in understanding CME initiation because it would increase the observational coverage of the solar surface magnetic field and the identification and tracking of magnetic regions would be possible for longer times (e.g., Mackay et al 2016). All these observations combined with a well-crafted modeling program will allow us to finally fully characterize the nature and evolution of pre-eruptive configurations towards eruption.

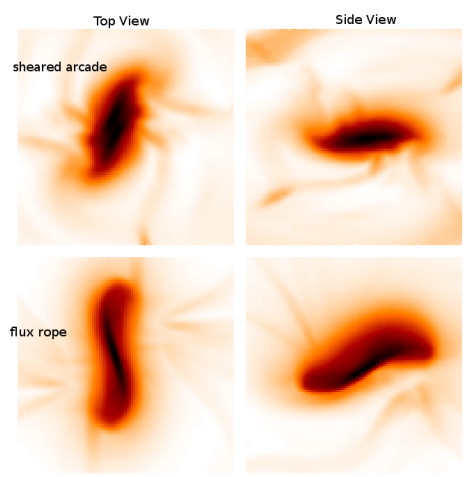

Fig. 21 LOS-integrations of $J^{2}$ for the two simulation snapshots of Figure 19 The left panels correspond to top views of the SMA and MFR in Figure 19 The right panels correspond to side views of the SMA and MFR. A reverse color-table is used.

\section{References}

Alexakis A, Mininni PD, Pouquet A (2006) On the Inverse Cascade of Magnetic Helicity. ApJ640:335-343, DOI 10.1086/500082, physics/0509069

Amari T, Démoulin P, Browning P, Hood A, Priest E (1991) The creation of the magnetic environment for prominence formation in a coronal arcade. A\&A241:604-612

Amari T, Luciani JF, Aly JJ, Tagger M (1996) Plasmoid formation in a single sheared arcade and application to coronal mass ejections. A\&A306:913

Amari T, Luciani JF, Mikic Z, Linker J (1999) Three-dimensional Solutions of Magnetohydrodynamic Equationsfor Prominence Magnetic Support: Twisted Magnetic Flux Rope. Astrophysical Journal Letters 518:L57-L60, DOI 10.1086/312053

Amari T, Luciani JF, Mikic Z, Linker J (2000) A Twisted Flux Rope Model for Coronal Mass Ejections and Two-Ribbon Flares. ApJL529:L49-L52, DOI 10.1086/312444

Amari T, Aly JJ, Mikic Z, Linker J (2007) Coronal Mass Ejection Initiation and Complex Topology Configurations in the Flux Cancellation and Breakout Models. ApJl 671:L189L192, DOI 10.1086/524942

Amari T, Canou A, Aly JJ, Delyon F, Alauzet F (2018) Magnetic cage and rope as the key for solar eruptions. Nature554:211-215, DOI 10.1038/nature24671

Antiochos SK (2013) Helicity Condensation as the Origin of Coronal and Solar Wind Structure. ApJ772:72, DOI 10.1088/0004-637X/772/1/72, 1211.4132

Antiochos SK, Klimchuk JA (1991) A model for the formation of solar prominences. ApJ378:372-377, DOI 10.1086/170437

Antiochos SK, Dahlburg RB, Klimchuk JA (1994) The magnetic field of solar prominences. ApJL420:L41-L44, DOI 10.1086/187158 
Antiochos SK, DeVore CR, Klimchuk JA (1999) A Model for Solar Coronal Mass Ejections. ApJ510:485-493, DOI 10.1086/306563, astro-ph/9807220

Antiochos SK, MacNeice PJ, Spicer DS (2000) The Thermal Nonequilibrium of Prominences. ApJ536:494-499, DOI 10.1086/308922

Archontis V (2008) Magnetic flux emergence in the Sun. Journal of Geophysical Research (Space Physics) 113(A12):3-+, DOI 10.1029/2007JA012422

Archontis V (2012) Magnetic flux emergence and associated dynamic phenomena in the Sun. Philosophical Transactions of the Royal Society of London Series A 370(1970):3088-3113, DOI $10.1098 /$ rsta.2012.0001

Archontis V, Hood AW (2009) Formation of Ellerman bombs due to 3D flux emergence. A\&A508:1469-1483, DOI 10.1051/0004-6361/200912455

Archontis V, Hood AW (2010) Flux emergence and coronal eruption. Astronomy and Astrophysics 514:A56+, DOI 10.1051/0004-6361/200913502

Archontis V, Hood AW (2012) Magnetic flux emergence: a precursor of solar plasma expulsion. A\&A537:A62, DOI 10.1051/0004-6361/201116956

Archontis V, Syntelis P (2019) The emergence of magnetic flux and its role on the onset of solar dynamic events. Philosophical Transactions of the Royal Society of London Series A 377(2148):20180,387, DOI 10.1098/rsta.2018.0387, 1904.06274

Archontis V, Török T (2008) Eruption of magnetic flux ropes during flux emergence. A\&A492:L35-L38, DOI 10.1051/0004-6361:200811131

Archontis V, Moreno-Insertis F, Galsgaard K, Hood A, O'Shea E (2004) Emergence of magnetic flux from the convection zone into the corona. A\&A426:1047-1063, DOI 10.1051/0004-6361: 20035934

Archontis V, Hood AW, Savcheva A, Golub L, Deluca E (2009) On the Structure and Evolution of Complexity in Sigmoids: A Flux Emergence Model. ApJ691:1276-1291, DOI 10.1088/ 0004-637X/691/2/1276

Archontis V, Hood AW, Tsinganos K (2013) The Emergence of Weakly Twisted Magnetic Fields in the Sun. ApJ778:42, DOI 10.1088/0004-637X/778/1/42

Archontis V, Hood AW, Tsinganos K (2014) Recurrent Explosive Eruptions and the "Sigmoidto-arcade" Transformation in the Sun Driven by Dynamical Magnetic Flux Emergence. ApJL786:L21, DOI 10.1088/2041-8205/786/2/L21

Aulanier G, Démoulin P (1998) 3-D magnetic configurations supporting prominences. I. The natural presence of lateral feet. A\&A329:1125-1137

Aulanier G, Démoulin P (2003) Amplitude and orientation of prominence magnetic fields from constant-alpha magnetohydrostatic models. A\&A402:769-780, DOI 10.1051/0004-6361: 20030227

Aulanier G, Schmieder B (2008) Magnetic helicity and solar prominence formation. In: Charbonnel C, Combes F, Samadi R (eds) SF2A-2008, p 543

Aulanier G, Démoulin P, Mein N, van Driel-Gesztelyi L, Mein P, Schmieder B (1999) 3-D magnetic configurations supporting prominences. III. Evolution of fine structures observed in a filament channel. A\&A342:867-880

Aulanier G, DeVore CR, Antiochos SK (2002) Prominence Magnetic Dips in ThreeDimensional Sheared Arcades. ApJL567:L97-L101, DOI 10.1086/339436

Aulanier G, Pariat E, Démoulin P (2005) Current sheet formation in quasi-separatrix layers and hyperbolic flux tubes. A\&A444(3):961-976, DOI 10.1051/0004-6361:20053600

Aulanier G, DeVore CR, Antiochos SK (2006a) Solar Prominence Merging. ApJ646(2):13491357, DOI 10.1086/505020

Aulanier G, Pariat E, Démoulin P, DeVore CR (2006b) Slip-Running Reconnection in QuasiSeparatrix Layers. Sol. Phys.238:347-376, DOI 10.1007/s11207-006-0230-2

Aulanier G, Török T, Démoulin P, DeLuca EE (2010) Formation of Torus-Unstable Flux Ropes and Electric Currents in Erupting Sigmoids. Astrophysics Journal 708:314-333, DOI 10.1088/0004-637X/708/1/314

Babcock HW, Babcock HD (1955) The Sun's Magnetic Field, 1952-1954. ApJ121:349, DOI 10.1086/145994

Baker D, van Driel-Gesztelyi L, Green LM (2012) Forecasting a CME by Spectroscopic Precursor? Sol. Phys.276:219-239, DOI 10.1007/s11207-011-9893-4

Ba̧k-Stȩślicka U, Gibson SE, Fan Y, Bethge C, Forland B, Rachmeler LA (2013) The Magnetic Structure of Solar Prominence Cavities: New Observational Signature Revealed by Coronal Magnetometry. ApJL770:L28, DOI 10.1088/2041-8205/770/2/L28, 1304.7388

Bein BM, Berkebile-Stoiser S, Veronig AM, Temmer M, Vršnak B (2012) Impulsive Accelera- 
tion of Coronal Mass Ejections. II. Relation to Soft X-Ray Flares and Filament Eruptions. ApJ755(1):44, DOI 10.1088/0004-637X/755/1/44, 1206.2144

Berger MA (1984) Rigorous new limits on magnetic helicity dissipation in the solar corona. Geophysical and Astrophysical Fluid Dynamics 30(1):79-104, DOI 10.1080/03091928408210078

Berger TE, Title AM (1996) On the Dynamics of Small-Scale Solar Magnetic Elements. ApJ463:365, DOI 10.1086/177250

Berkebile-Stoiser S, Veronig AM, Bein BM, Temmer M (2012) Relation between the Coronal Mass Ejection Acceleration and the Non-thermal Flare Characteristics. ApJ753(1):88, DOI 10.1088/0004-637X/753/1/88

Bernasconi PN, Rust DM, Georgoulis MK, Labonte BJ (2002) Moving Dipolar Features in an Emerging Flux Region. Sol. Phys.209:119-139, DOI 10.1023/A:1020943816174

Biskamp D (1993) Nonlinear magnetohydrodynamics. Cambridge University Press

Bobra MG, van Ballegooijen AA, DeLuca EE (2008) Modeling Nonpotential Magnetic Fields in Solar Active Regions. ApJ672:1209-1220, DOI 10.1086/523927

Bommier V, Leroy JL (1998) Global Pattern of the Magnetic Field Vectors Above Neutral Lines from 1974 to 1982: Pic-du-Midi Observations of Prominences. In: Webb DF, Schmieder B, Rust DM (eds) IAU Colloq. 167: New Perspectives on Solar Prominences, Astronomical Society of the Pacific Conference Series, vol 150, p 434

Bommier V, Landi Degl'Innocenti E, Leroy JL, Sahal-Brechot S (1994) Complete determination of the magnetic field vector and of the electron density in 14 prominences from linear polarization measurements in the HeI D3 and H-alpha lines. Sol. Phys.154:231-260, DOI 10.1007/BF00681098

Brandenburg A, Subramanian K (2005) Astrophysical magnetic fields and nonlinear dynamo theory. Physics Reports417:1-209, DOI 10.1016/j.physrep.2005.06.005, astro-ph/0405052

Burlaga L, Sittler E, Mariani F, Schwenn R (1981) Magnetic loop behind an interplanetary shock - Voyager, Helios, and IMP 8 observations. JGR86:6673-6684, DOI 10.1029/ JA086iA08p06673

Cameron R, Schüssler M, Vögler A, Zakharov V (2007) Radiative magnetohydrodynamic simulations of solar pores. A\&A474(1):261-272, DOI 10.1051/0004-6361:20078140

Canou A, Amari T, Bommier V, Schmieder B, Aulanier G, Li H (2009) Evidence for a PreEruptive Twisted Flux Rope Using the Themis Vector Magnetograph. ApJL693:L27-L30, DOI 10.1088/0004-637X/693/1/L27

Chae J, Moon YJ, Pevtsov AA (2004) Observational Evidence of Magnetic Flux Submergence in Flux Cancellation Sites. ApJL602:L65-L68, DOI 10.1086/382222

Chandra R, Mandrini CH, Schmieder B, Joshi B, Cristiani GD, Cremades H, Pariat E, Nuevo FA, Srivastava AK, Uddin W (2017) Blowout jets and impulsive eruptive flares in a baldpatch topology. A\&A598:A41, DOI 10.1051/0004-6361/201628984, 1610.01918

Chen B, Bastian TS, Gary DE (2014) Direct Evidence of an Eruptive, Filament-hosting Magnetic Flux Rope Leading to a Fast Solar Coronal Mass Ejection. ApJ794:149, DOI 10.1088/0004-637X/794/2/149, 1408.6473

Chen F, Rempel M, Fan Y (2017) Emergence of Magnetic Flux Generated in a Solar Convective Dynamo. I. The Formation of Sunspots and Active Regions, and The Origin of Their Asymmetries. ApJ846(2):149, DOI 10.3847/1538-4357/aa85a0, 1704.05999

Chen J (1989) Effects of toroidal forces in current loops embedded in a background plasma. ApJ338:453-470, DOI 10.1086/167211

Chen PF (2011) Coronal Mass Ejections: Models and Their Observational Basis. Living Reviews in Solar Physics 8:1, DOI 10.12942/lrsp-2011-1

Chen Y, Du G, Zhao D, Wu Z, Liu W, Wang B, Ruan G, Feng S, Song H (2016) Imaging a Magnetic-breakout Solar Eruption. ApJL820:L37, DOI 10.3847/2041-8205/820/2/L37

Cheng X, Ding MD (2016) The Characteristics of the Footpoints of Solar Magnetic Flux Ropes during Eruptions. ApJS225:16, DOI 10.3847/0067-0049/225/1/16, 1605.04047

Cheng X, Zhang J, Liu Y, Ding MD (2011) Observing Flux Rope Formation During the Impulsive Phase of a Solar Eruption. ApJL732:L25, DOI 10.1088/2041-8205/732/2/L25, 1103.5084

Cheng X, Zhang J, Ding MD, Liu Y, Poomvises W (2013) The Driver of Coronal Mass Ejections in the Low Corona: A Flux Rope. ApJ763:43, DOI 10.1088/0004-637X/763/1/43, 1211.6524

Cheng X, Ding MD, Guo Y, Zhang J, Vourlidas A, Liu YD, Olmedo O, Sun JQ, Li C (2014a) Tracking the Evolution of a Coherent Magnetic Flux Rope Continuously from the Inner to the Outer Corona. ApJ780:28, DOI 10.1088/0004-637X/780/1/28, 1310.6782

Cheng X, Ding MD, Zhang J, Sun XD, Guo Y, Wang YM, Kliem B, Deng YY (2014b) 
Formation of a Double-decker Magnetic Flux Rope in the Sigmoidal Solar Active Region 11520. ApJ789(2):93, DOI 10.1088/0004-637X/789/2/93, 1405.4923

Cheng X, Ding MD, Fang C (2015) Imaging and Spectroscopic Diagnostics on the Formation of Two Magnetic Flux Ropes Revealed by SDO/AIA and IRIS. ApJ804(2):82, DOI 10.1088/ 0004-637X/804/2/82, 1502.07801

Cheng X, Guo Y, Ding M (2017) Origin and Structures of Solar Eruptions I: Magnetic Flux Rope. Science in China Earth Sciences 60:1383-1407, DOI 10.1007/s11430-017-9074-6, 1705.08198

Cheng X, Zhang J, Kliem B, Török T, Xing C, Zhou ZJ, Inhester B, Ding MD (2020) Initiation and Early Kinematic Evolution of Solar Eruptions. ApJ894(2):85, DOI 10.3847/1538-4357/ ab886a, 2004.03790

Cheung MCM, Isobe H (2014) Flux Emergence (Theory). Living Reviews in Solar Physics 11(1):3, DOI 10.12942/lrsp-2014-3

Cheung MCM, Rempel M, Title AM, Schüssler M (2010) Simulation of the Formation of a Solar Active Region. ApJ720:233-244, DOI 10.1088/0004-637X/720/1/233

Cheung MCM, Rempel M, Chintzoglou G, Chen F, Testa P, Martínez-Sykora J, Sainz Dalda A, DeRosa ML, Malanushenko A, Hansteen V, De Pontieu B, Carlsson M, Gudiksen B, McIntosh SW (2019) A comprehensive three-dimensional radiative magnetohydrodynamic simulation of a solar flare. Nature Astronomy 3:160-166, DOI 10.1038/s41550-018-0629-3

Chintzoglou G, Patsourakos S, Vourlidas A (2015) Formation of Magnetic Flux Ropes during a Confined Flaring Well before the Onset of a Pair of Major Coronal Mass Ejections. ApJ809:34, DOI 10.1088/0004-637X/809/1/34, 1507.01165

Chintzoglou G, Zhang J, Cheung MCM, Kazachenko M (2019) The Origin of Major Solar Activity: Collisional Shearing between Nonconjugated Polarities of Multiple Bipoles Emerging within Active Regions. ApJ871:67, DOI 10.3847/1538-4357/aaef30, 1811.02186

Choe GS, Lee LC (1992) Formation of Solar Prominences by Photospheric Shearing Motions. Sol. Phys.138(2):291-329, DOI 10.1007/BF00151917

Dalmasse K, Aulanier G, Démoulin P, Kliem B, Török T, Pariat E (2015) The Origin of Net Electric Currents in Solar Active Regions. ApJ810:17, DOI 10.1088/0004-637X/810/1/17, 1507.05060

Danilovic S, Solanki SK, Barthol P, Gandorfer A, Gizon L, Hirzberger J, Riethmüller TL, van Noort M, Blanco Rodríguez J, Del Toro Iniesta JC, Orozco Suárez D, Schmidt W, Martínez Pillet V, Knölker M (2017) Photospheric Response to an Ellerman Bomb-like Event-An Analogy of Sunrise/IMaX Observations and MHD Simulations. The Astrophysical Journal Supplement Series 229:5, DOI 10.3847/1538-4365/229/1/5

Démoulin P, Aulanier G (2010) Criteria for Flux Rope Eruption: Non-equilibrium Versus Torus Instability. ApJ718:1388-1399, DOI 10.1088/0004-637X/718/2/1388, 1006.1785

Démoulin P, Hénoux JC, Priest ER, Mandrini CH (1996) Quasi-Separatrix layers in solar flares. I. Method. A\&A308:643-655

Dere KP, Brueckner GE, Howard RA, Koomen MJ, Korendyke CM, Kreplin RW, Michels DJ, Moses JD, Moulton NE, Socker DG, St Cyr OC, Delaboudinière JP, Artzner GE, Brunaud J, Gabriel AH, Hochedez JF, Millier F, Song XY, Chauvineau JP, Marioge JP, Defise JM, Jamar C, Rochus P, Catura RC, Lemen JR, Gurman JB, Neupert W, Clette F, Cugnon P, van Dessel EL, Lamy PL, Llebaria A, Schwenn R, Simnett GM (1997) EIT and LASCO Observations of the Initiation of a Coronal Mass Ejection. Sol. Phys.175:601-612, DOI 10.1023/A:1004907307376

DeVore CR, Antiochos SK (2000) Dynamical Formation and Stability of Helical Prominence Magnetic Fields. ApJ539:954-963, DOI 10.1086/309275

DeVore CR, Antiochos SK, Aulanier G (2005) Solar Prominence Interactions. ApJ629:11221134, DOI 10.1086/431721

Dhakal SK, Chintzoglou G, Zhang J (2018) A Study of a Compound Solar Eruption with Two Consecutive Erupting Magnetic Structures. ApJ860(1):35, DOI 10.3847/1538-4357/aac028, 1807.00206

Duan A, Jiang C, He W, Feng X, Zou P, Cui J (2019) A Study of Pre-flare Solar Coronal Magnetic Fields: Magnetic Flux Ropes. ApJ884(1):73, DOI 10.3847/1538-4357/ab3e33, 1908.08643

Dudík J, Aulanier G, Schmieder B, Bommier V, Roudier T (2008) Topological Departures from Translational Invariance along a Filament Observed by THEMIS. Sol. Phys.248:2950, DOI 10.1007/s11207-008-9155-2

Fan Y (2001) The Emergence of a Twisted $\Omega$-Tube into the Solar Atmosphere. ApJL554:L111- 
L114, DOI 10.1086/320935

Fan Y (2009a) Magnetic Fields in the Solar Convection Zone. Living Reviews in Solar Physics 6(1):4, DOI 10.12942/lrsp-2009-4

Fan Y (2009b) The Emergence of a Twisted Flux Tube into the Solar Atmosphere: Sunspot Rotations and the Formation of a Coronal Flux Rope. ApJ697:1529-1542, DOI 10.1088/ 0004-637X/697/2/1529

Fan Y (2018) MHD Simulation of Prominence Eruption. ApJ862(1):54, DOI 10.3847/ $1538-4357 /$ aaccee, 1806.06305

Fan Y, Gibson SE (2004) Numerical Simulations of Three-dimensional Coronal Magnetic Fields Resulting from the Emergence of Twisted Magnetic Flux Tubes. ApJ609:1123-1133, DOI $10.1086 / 421238$

Fang F, Fan Y (2015) $\delta$-Sunspot Formation in Simulation of Active-region-scale Flux Emergence. ApJ806(1):79, DOI 10.1088/0004-637X/806/1/79, 1504.04393

Fang F, Manchester W, Abbett WP, van der Holst B (2010) Simulation of Flux Emergence from the Convection Zone to the Corona. ApJ714:1649-1657, DOI 10.1088/0004-637X/714/ $2 / 1649$

Fang F, Manchester W IV, Abbett WP, van der Holst B (2012) Buildup of Magnetic Shear and Free Energy during Flux Emergence and Cancellation. Astrophysical Journal 754:15, DOI 10.1088/0004-637X/754/1/15, 1205.3764

Fang F, Fan Y, McIntosh SW (2014) Rotating Solar Jets in Simulations of Flux Emergence with Thermal Conduction. ApJL789:L19, DOI 10.1088/2041-8205/789/1/L19

Fisher GH, Abbett WP, Bercik DJ, Kazachenko MD, Lynch BJ, Welsch BT, Hoeksema JT, Hayashi K, Liu Y, Norton AA, Dalda AS, Sun X, DeRosa ML, Cheung MCM (2015) The Coronal Global Evolutionary Model: Using HMI Vector Magnetogram and Doppler Data to Model the Buildup of Free Magnetic Energy in the Solar Corona. Space Weather 13(6):369373, DOI 10.1002/2015SW001191, 1505.06018

Foley CR, Harra LK, Culhane JL, Mason KO (2001) Eruption of a Flux Rope on the Disk of the Sun: Evidence for the Coronal Mass Ejection Trigger? ApJL560(1):L91-L94, DOI $10.1086 / 324059$

Forland BC, Gibson SE, Dove JB, Rachmeler LA, Fan Y (2013) Coronal Cavity Survey: Morphological Clues to Eruptive Magnetic Topologies. Sol. Phys.288:603-615, DOI 10.1007/s11207-013-0361-1

Fu HS, Vaivads A, Khotyaintsev YV, André M, Cao JB, Olshevsky V, Eastwood JP, Retinò A (2017) Intermittent energy dissipation by turbulent reconnection. GRL44:37-43, DOI 10.1002/2016GL071787

Gaizauskas V (2002) Formation of a Switchback During the Rising Phase of Solar Cycle 21. Sol. Phys.211:179-188, DOI 10.1023/A:1022579419832

Gaizauskas V, Zirker JB, Sweetland C, Kovacs A (1997) Formation of a Solar Filament Channel. ApJ479:448, DOI 10.1086/512788

Gaizauskas V, Mackay DH, Harvey KL (2001) Evolution of Solar Filament Channels Observed during a Major Poleward Surge of Photospheric Magnetic Flux. ApJ558:888-902, DOI $10.1086 / 322298$

Georgoulis MK, Titov VS, Mikić Z (2012a) Non-neutralized Electric Current Patterns in Solar Active Regions: Origin of the Shear-generating Lorentz Force. ApJ761(1):61, DOI 10.1088/ 0004-637X/761/1/61, 1210.2919

Georgoulis MK, Tziotziou K, Raouafi NE (2012b) Magnetic Energy and Helicity Budgets in the Active-region Solar Corona. II. Nonlinear Force-free Approximation. ApJ759:1, DOI $10.1088 / 0004-637 \mathrm{X} / 759 / 1 / 1,1209.5606$

Georgoulis MK, Nindos A, Zhang H (2019) The source and engine of coronal mass ejections. Philosophical Transactions of the Royal Society of London Series A 377(2148):20180,094, DOI 10.1098/rsta.2018.0094

Gibb GPS, Mackay DH, Green LM, Meyer KA (2014) Simulating the Formation of a Sigmoidal Flux Rope in AR10977 from SOHO/MDI Magnetograms. ApJ782:71, DOI 10.1088/0004-637X/782/2/71

Gibson S (2015) Coronal Cavities: Observations and Implications for the Magnetic Environment of Prominences, Astrophysics and Space Science Library, vol 415, Springer, p 323. DOI 10.1007/978-3-319-10416-4_13

Gibson SE, Fan Y (2006a) Coronal prominence structure and dynamics: A magnetic flux rope interpretation. Journal of Geophysical Research (Space Physics) 111(A12):A12103, DOI 10.1029/2006JA011871 
Gibson SE, Fan Y (2006b) The Partial Expulsion of a Magnetic Flux Rope. ApJL637:L65-L68, DOI $10.1086 / 500452$

Gibson SE, Kucera TA, Rastawicki D, Dove J, de Toma G, Hao J, Hill S, Hudson HS, Marqué C, McIntosh PS, Rachmeler L, Reeves KK, Schmieder B, Schmit DJ, Seaton DB, Sterling AC, Tripathi D, Williams DR, Zhang M (2010) Three-dimensional Morphology of a Coronal Prominence Cavity. ApJ724:1133-1146, DOI 10.1088/0004-637X/724/2/1133

Gilbert HR, Holzer TE, Burkepile JT (2001) Observational Interpretation of an Active Prominence on 1999 May 1. ApJ549:1221-1230, DOI 10.1086/319444

Green LM, Kliem B (2009) Flux Rope Formation Preceding Coronal Mass Ejection Onset. ApJL700:L83-L87, DOI 10.1088/0004-637X/700/2/L83, 0906.4794

Green LM, Kliem B (2014) Observations of flux rope formation prior to coronal mass ejections. In: Schmieder B, Malherbe JM, Wu ST (eds) Nature of Prominences and their Role in Space Weather, IAU Symposium, vol 300, pp 209-214, DOI 10.1017/S1743921313010983, 1312.4388

Green LM, Kliem B, Wallace AJ (2011) Photospheric flux cancellation and associated flux rope formation and eruption. A\&A526:A2, DOI 10.1051/0004-6361/201015146, 1011.1227

Green LM, Valori G, Zuccarello FP, Zharkov S, Matthews SA, Guglielmino SL (2017) The 2013 February 17 Sunquake in the Context of the Active Region's Magnetic Field Configuration. ApJ849:40, DOI 10.3847/1538-4357/aa8db6, 1709.04874

Green LM, Török T, Vršnak B, Manchester W, Veronig A (2018) The Origin, Early Evolution and Predictability of Solar Eruptions. Space Sci. Rev.214:46, DOI 10.1007/ s11214-017-0462-5, 1801.04608

Gunár S, Dudík J, Aulanier G, Schmieder B, Heinzel P (2018) Importance of the H $\alpha$ Visibility and Projection Effects for the Interpretation of Prominence Fine-structure Observations. ApJ867:115, DOI 10.3847/1538-4357/aae4e1

Guo Y, Schmieder B, Démoulin P, Wiegelmann T, Aulanier G, Török T, Bommier V (2010) Coexisting Flux Rope and Dipped Arcade Sections Along One Solar Filament. ApJ714:343354, DOI 10.1088/0004-637X/714/1/343

Guo Y, Ding MD, Cheng X, Zhao J, Pariat E (2013) Twist Accumulation and Topology Structure of a Solar Magnetic Flux Rope. ApJ779:157, DOI 10.1088/0004-637X/779/2/157, 1311.1883

Hagyard MJ, Smith JB Jr, Teuber D, West EA (1984) A quantitative study relating observed shear in photospheric magnetic fields to repeated flaring. Sol. Phys.91:115-126, DOI 10. 1007 /BF00213618

Hanaoka Y, Sakurai T (2017) Statistical Study of the Magnetic Field Orientation in Solar Filaments. ApJ851:130, DOI 10.3847/1538-4357/aa9cf1, 1711.07735

Hansteen V, Ortiz A, Archontis V, Carlsson M, Pereira TMD, Bjørgen JP (2019) Ellerman bombs and UV bursts: transient events in chromospheric current sheets. A\&A626:A33, DOI 10.1051/0004-6361/201935376, 1904.11524

Hansteen VH, Archontis V, Pereira TMD, Carlsson M, Rouppe van der Voort L, Leenaarts J (2017) Bombs and Flares at the Surface and Lower Atmosphere of the Sun. ApJ839:22, DOI 10.3847/1538-4357/aa6844

Harra LK, Matthews S, Culhane JL, Cheung MCM, Kontar EP, Hara H (2013) The Location of Non-thermal Velocity in the Early Phases of Large Flares-Revealing Pre-eruption Flux Ropes. ApJ774(2):122, DOI 10.1088/0004-637X/774/2/122

Harvey KL, Jones HP, Schrijver CJ, Penn MJ (1999) Does Magnetic Flux Submerge at Flux Cancelation Sites? Sol. Phys.190:35-44, DOI 10.1023/A:1005237719407

Hood AW, Priest ER (1981) Critical conditions for magnetic instabilities in force-free coronal loops. Geophysical and Astrophysical Fluid Dynamics 17:297-318, DOI 10.1080/ 03091928108243687

Hood AW, Archontis V, Galsgaard K, Moreno-Insertis F (2009) The emergence of toroidal flux tubes from beneath the solar photosphere. A\&A503:999-1011, DOI 10.1051/0004-6361/ 200912189

Hood AW, Archontis V, MacTaggart D (2012) 3D MHD Flux Emergence Experiments: Idealised Models and Coronal Interactions. Sol. Phys.278(1):3-31, DOI 10.1007/ s11207-011-9745-2, 1103.3685

Hu Q, Dasgupta B (2008) An Improved Approach to Non-Force-Free Coronal Magnetic Field Extrapolation. Sol. Phys.247(1):87-101, DOI 10.1007/s11207-007-9090-7

Illing RME, Hundhausen AJ (1985) Observation of a coronal transient from 1.2 to 6 solar radii. JGR90:275-282, DOI 10.1029/JA090iA01p00275 
Inoue S, Hayashi K, Kusano K (2016) Structure and Stability of Magnetic Fields in Solar Active Region 12192 Based on the Nonlinear Force-free Field Modeling. ApJ818:168, DOI $10.3847 / 0004-637 \mathrm{X} / 818 / 2 / 168,1601.00791$

Inoue S, Shiota D, Bamba Y, Park SH (2018) Magnetohydrodynamic Modeling of a Solar Eruption Associated with an X9.3 Flare Observed in the Active Region 12673. ApJ867(1):83, DOI $10.3847 / 1538-4357 /$ aae079, 1809.02309

Isobe H, Tripathi D, Asai A, Jain R (2007) Large-Amplitude Oscillation of an Erupting Filament as Seen in EUV, H $\alpha$, and Microwave Observations. Sol. Phys.246:89-99, DOI 10.1007/s11207-007-9091-6

Isobe H, Proctor MRE, Weiss NO (2008) Convection-driven Emergence of Small-Scale Magnetic Fields and their Role in Coronal Heating and Solar Wind Acceleration. ApJL679:L57, DOI $10.1086 / 589150$

James AW, Valori G, Green LM, Liu Y, Cheung MCM, Guo Y, van Driel-Gesztelyi L (2018) An Observationally Constrained Model of a Flux Rope that Formed in the Solar Corona. ApJL855:L16, DOI 10.3847/2041-8213/aab15d, 1802.07965

Jiang C, Wu ST, Feng X, Hu Q (2014) Formation and Eruption of an Active Region Sigmoid. I. A Study by Nonlinear Force-free Field Modeling. ApJ780:55, DOI 10.1088/0004-637X/ $780 / 1 / 55,1310.8196$

Jiang C, Wu ST, Yurchyshyn V, Wang H, Feng X, Hu Q (2016) How Did a Major Confined Flare Occur in Super Solar Active Region 12192? ApJ828:62, DOI 10.3847/0004-637X/828/ $1 / 62,1606.09334$

Joshi NC, Sterling AC, Moore RL, Magara T, Moon YJ (2017) Onset of a large ejective solar eruption from a typical coronal-jet-base field configuration. ApJ845:26, DOI 10.3847/ $1538-4357 /$ aa7c1b, 1706.09176

Kaneko T, Yokoyama T (2018) Impact of Dynamic State on the Mass Condensation Rate of Solar Prominences. ApJ869(2):136, DOI 10.3847/1538-4357/aaee6f, 1811.00828

Karna N, Pesnell WD, Zhang J (2015a) Appearances and Statistics of Coronal Cavities During the Ascending Phase of Solar Cycle 24. ApJ810:123, DOI 10.1088/0004-637X/810/2/123

Karna N, Zhang J, Pesnell WD, Hess Webber SA (2015b) Study of the 3D Geometric Structure and Temperature of a Coronal Cavity Using the Limb Synoptic Map Method. ApJ810:124, DOI 10.1088/0004-637X/810/2/124

Karpen JT, Antiochos SK, Hohensee M, Klimchuk JA, MacNeice PJ (2001) Are Magnetic Dips Necessary for Prominence Formation? ApJL553:L85-L88, DOI 10.1086/320497

Karpen JT, Antiochos SK, Klimchuk JA (2006) The Origin of High-Speed Motions and Threads in Prominences. ApJ637:531-540, DOI 10.1086/498237

Karpen JT, Antiochos SK, DeVore CR (2012) The Mechanisms for the Onset and Explosive Eruption of Coronal Mass Ejections and Eruptive Flares. ApJ760:81, DOI 10.1088/ 0004-637X/760/1/81

Kliem B, Török T (2006) Torus Instability. Physical Review Letters 96(25):255,002-+, DOI 10.1103/PhysRevLett.96.255002, arXiv: physics/0605217

Kliem B, Su YN, van Ballegooijen AA, DeLuca EE (2013) Magnetohydrodynamic Modeling of the Solar Eruption on 2010 April 8. ApJ779:129, DOI 10.1088/0004-637X/779/2/129, 1304.6981

Kliem B, Lin J, Forbes TG, Priest ER, Török T (2014a) Catastrophe versus Instability for the Eruption of a Toroidal Solar Magnetic Flux Rope. ApJ789:46, DOI 10.1088/0004-637X/ $789 / 1 / 46,1404.5922$

Kliem B, Török T, Titov VS, Lionello R, Linker JA, Liu R, Liu C, Wang H (2014b) Slow Rise and Partial Eruption of a Double-decker Filament. II. A Double Flux Rope Model. ApJ792:107, DOI 10.1088/0004-637X/792/2/107, 1407.2272

Kliem B, Lee J, White SM, Liu C, Masuda S (2020) Flux Rope Formation by a Confined Solar Flare Preceding a Coronal Mass Ejection. ApJ, submitted

Klimchuk JA (1990) Shear-induced inflation of coronal magnetic fields. ApJ354:745-754, DOI $10.1086 / 168731$

Klimchuk JA (2006) On Solving the Coronal Heating Problem. Sol. Phys.234:41-77, DOI 10.1007/s11207-006-0055-z, astro-ph/0511841

Klimchuk JA, Sturrock PA, Yang WH (1988) Coronal magnetic fields produced by photospheric shear. ApJ335:456-467, DOI 10.1086/166939

Knizhnik KJ, Antiochos SK, DeVore CR (2015) Filament Channel Formation via Magnetic Helicity Condensation. ApJ809:137, DOI 10.1088/0004-637X/809/2/137, 1411.5396

Knizhnik KJ, Antiochos SK, DeVore CR, Wyper PF (2017) The Mechanism for the En- 
ergy Buildup Driving Solar Eruptive Events. ApJL851:L17, DOI 10.3847/2041-8213/aa9e0a, 1711.00166

Knizhnik KJ, Linton MG, DeVore CR (2018) The Role of Twist in Kinked Flux Rope Emergence and Delta-spot Formation. ApJ864(1):89, DOI 10.3847/1538-4357/aad68c, 1808. 05562

Kontogiannis I, Georgoulis MK, Park SH, Guerra JA (2017) Non-neutralized Electric Currents in Solar Active Regions and Flare Productivity. Sol. Phys.292:159, DOI 10.1007/ s11207-017-1185-1, 1708.07087

Kuckein C, Martínez Pillet V, Centeno R (2012) An active region filament studied simultaneously in the chromosphere and photosphere. I. Magnetic structure. A\&A539:A131, DOI 10.1051/0004-6361/201117675, 1112.1672

Kumar P, Yurchyshyn V, Cho KS, Wang H (2017) Multiwavelength observations of a flux rope formation by series of magnetic reconnection in the chromosphere. A\&A603:A36, DOI 10.1051/0004-6361/201629295, 1703.09871

Kuperus M, Raadu MA (1974) The Support of Prominences Formed in Neutral Sheets. A\&A31:189

LaBonte BJ, Georgoulis MK, Rust DM (2007) Survey of Magnetic Helicity Injection in Regions Producing X-Class Flares. ApJ671:955-963, DOI 10.1086/522682

Leake JE, Arber TD (2006) The emergence of magnetic flux through a partially ionised solar atmosphere. A\&A450:805-818, DOI 10.1051/0004-6361:20054099

Leake JE, Linton MG, Török T (2013) Simulations of Emerging Magnetic Flux. I. The Formation of Stable Coronal Flux Ropes. ApJ778:99, DOI 10.1088/0004-637X/778/2/99, 1308.6204

Leake JE, Linton MG, Antiochos SK (2014) Simulations of Emerging Magnetic Flux. II. The Formation of Unstable Coronal Flux Ropes and the Initiation of Coronal Mass Ejections. ApJ787:46, DOI 10.1088/0004-637X/787/1/46

Lee EJ, Archontis V, Hood AW (2015) Helical Blowout Jets in the Sun: Untwisting and Propagation of Waves. ApJL798:L10, DOI 10.1088/2041-8205/798/1/L10

Leenaarts J (2020) Radiation hydrodynamics in simulations of the solar atmosphere. Living Reviews in Solar Physics 17(1):3, DOI 10.1007/s41116-020-0024-x, 2002.03623

Leroy JL, Bommier V, Sahal-Brechot S (1983) The magnetic field in the prominences of the polar crown. Sol. Phys.83:135-142, DOI 10.1007/BF00148248

Leroy JL, Bommier V, Sahal-Brechot S (1984) New data on the magnetic structure of quiescent prominences. A\&A131:33-44

Li LP, Peter H, Chen F, Zhang J (2014) Conversion from mutual helicity to self-helicity observed with IRIS. A\&A570:A93, DOI 10.1051/0004-6361/201424377, 1410.5597

Li X, Morgan H, Leonard D, Jeska L (2012) A Solar Tornado Observed by AIA/SDO: Rotational Flow and Evolution of Magnetic Helicity in a Prominence and Cavity. ApJL752:L22, DOI 10.1088/2041-8205/752/2/L22, 1205.3819

Lin J, Forbes TG (2000) Effects of reconnection on the coronal mass ejection process. JGR105:2375-2392, DOI 10.1029/1999JA900477

Lin J, Raymond JC, van Ballegooijen AA (2004) The Role of Magnetic Reconnection in the Observable Features of Solar Eruptions. ApJ602:422-435, DOI 10.1086/380900

Lin Y, Engvold OR, Wiik JE (2003) Counterstreaming in a Large Polar Crown Filament. Sol. Phys.216:109-120, DOI 10.1023/A:1026150809598

Lin Y, Engvold O, Rouppe van der Voort LHM (2012) Small-scale, Dynamic Bright Blobs in Solar Filaments and Active Regions. ApJ747:129, DOI 10.1088/0004-637X/747/2/129, 1201.0406

Linker JA, Lionello R, Mikić Z, Amari T (2001) Magnetohydrodynamic modeling of prominence formation within a helmet streamer. Journal of Geophysical Research 106:25,16525,176, DOI 10.1029/2000JA004020

Lionello R, Linker JA, Mikić Z (2001) Including the Transition Region in Models of the LargeScale Solar Corona. Astrophysical Jounal 546:542-551, DOI 10.1086/318254

Lionello R, Mikić Z, Linker JA, Amari T (2002) Magnetic Field Topology in Prominences. ApJ581:718-725, DOI 10.1086/344222

Lites BW (2005) Magnetic Flux Ropes in the Solar Photosphere: The Vector Magnetic Field under Active Region Filaments. ApJ622:1275-1291, DOI 10.1086/428080

Lites BW, Low BC, Martinez Pillet V, Seagraves P, Skumanich A, Frank ZA, Shine RA, Tsuneta S (1995) The Possible Ascent of a Closed Magnetic System through the Photosphere. ApJ446:877, DOI 10.1086/175845 
Lites BW, Kubo M, Berger T, Frank Z, Shine R, Tarbell T, Title A, Okamoto TJ, Otsuji K (2010) Emergence of Helical Flux and the Formation of an Active Region Filament Channel. ApJ718:474-487, DOI 10.1088/0004-637X/718/1/474

Liu L, Cheng X, Wang Y, Zhou Z, Guo Y, Cui J (2018) Rapid Buildup of a Magnetic Flux Rope during a Confined X2.2 Class Flare in NOAA AR 12673. ApJL867(1):L5, DOI 10. $3847 / 2041-8213 /$ aae826, 1810.04424

Liu R (2020) Magnetic Flux Ropes in the Solar Corona: Structure and Evolution toward Eruption. arXiv e-prints arXiv:2007.11363, 2007.11363

Liu R, Liu C, Wang S, Deng N, Wang H (2010) Sigmoid-to-flux-rope Transition Leading to a Loop-like Coronal Mass Ejection. ApJL725:L84-L90, DOI 10.1088/2041-8205/725/1/L84, 1011.1181

Liu R, Kliem B, Török T, Liu C, Titov VS, Lionello R, Linker JA, Wang H (2012) Slow Rise and Partial Eruption of a Double-decker Filament. I. Observations and Interpretation. ApJ756:59, DOI 10.1088/0004-637X/756/1/59, 1207.1757

Liu R, Kliem B, Titov VS, Chen J, Wang Y, Wang H, Liu C, Xu Y, Wiegelmann T (2016) Structure, Stability, and Evolution of Magnetic Flux Ropes from the Perspective of Magnetic Twist. ApJ818:148, DOI 10.3847/0004-637X/818/2/148, 1512.02338

Liu Y, Sun X, Török T, Titov VS, Leake JE (2017) Electric-current Neutralization, Magnetic Shear, and Eruptive Activity in Solar Active Regions. ApJL846:L6, DOI 10.3847/2041-8213/ aa861e, 1708.04411

Livi SHB, Wang J, Martin SF (1985) The cancellation of magnetic flux. I - On the quiet sun. Australian Journal of Physics 38:855-873, DOI 10.1071/PH850855

Longcope DW, Klapper I (1997) Dynamics of a Thin Twisted Flux Tube. ApJ488:443-453, DOI $10.1086 / 304680$

Low BC, Hundhausen JR (1995) Magnetostatic structures of the solar corona. 2: The magnetic topology of quiescent prominences. ApJ443:818-836, DOI 10.1086/175572

Luna M, Karpen JT, DeVore CR (2012) Formation and Evolution of a Multi-threaded Solar Prominence. ApJ746:30, DOI 10.1088/0004-637X/746/1/30, 1201.3559

Lynch BJ, Antiochos SK, DeVore CR, Luhmann JG, Zurbuchen TH (2008) Topological Evolution of a Fast Magnetic Breakout CME in Three Dimensions. Astrophyiscal Journal 683:1192-1206, DOI 10.1086/589738

Mackay DH (2015) Formation and Large-Scale Patterns of Filament Channels and Filaments. In: Vial JC, Engvold O (eds) Solar Prominences, Astrophysics and Space Science Library, vol 415, p 355, DOI 10.1007/978-3-319-10416-4\_14

Mackay DH, van Ballegooijen AA (2005) New Results in Modeling the Hemispheric Pattern of Solar Filaments. ApJL621:L77-L80, DOI 10.1086/428904

Mackay DH, Gaizauskas V, Yeates AR (2008) Where Do Solar Filaments Form?: Consequences for Theoretical Models. Sol. Phys.248:51-65, DOI 10.1007/s11207-008-9127-6

Mackay DH, Karpen JT, Ballester JL, Schmieder B, Aulanier G (2010) Physics of Solar Prominences: II Magnetic Structure and Dynamics. Space Sci. Rev.151:333-399, DOI 10.1007/s11214-010-9628-0, 1001.1635

Mackay DH, Green LM, van Ballegooijen A (2011) Modeling the Dispersal of an Active Region: Quantifying Energy Input into the Corona. ApJ729:97, DOI 10.1088/0004-637X/729/2/97, 1102.5296

Mackay DH, Gaizauskas V, Yeates AR (2014) Where Do Solar Filaments Form? In: Schmieder B, Malherbe JM, Wu ST (eds) Nature of Prominences and their Role in Space Weather, IAU Symposium, vol 300, pp 445-446, DOI 10.1017/S1743921313011484

Mackay DH, Yeates AR, Bocquet FX (2016) Impact of an L5 Magnetograph on Nonpotential Solar Global Magnetic Field Modeling. ApJ825:131, DOI 10.3847/0004-637X/825/2/131

Mackay DH, DeVore CR, Antiochos SK, Yeates AR (2018) Magnetic Helicity Condensation and the Solar Cycle. ApJ869:62, DOI 10.3847/1538-4357/aaec7c

MacTaggart D, Hood AW (2009) On the emergence of toroidal flux tubes: general dynamics and comparisons with the cylinder model. Astronomy and Astrophysics 507:995-1004, DOI 10.1051/0004-6361/200912930, 0909.3987

Magara T (2011) A Possible Mechanism of Flux Cancellation via U-Loop Emergence on the Sun. PASJ 63:417-426, DOI 10.1093/pasj/63.2.417

Magara T, Longcope DW (2001) Sigmoid Structure of an Emerging Flux Tube. ApJL559:L55L59, DOI 10.1086/323635

Magara T, Longcope DW (2003) Injection of Magnetic Energy and Magnetic Helicity into the Solar Atmosphere by an Emerging Magnetic Flux Tube. ApJ586:630-649, DOI 10.1086/ 
367611

Manchester W, Kilpua EKJ, Liu YD, Lugaz N, Riley P, Török T, Vršnak B (2017) The Physical Processes of CME/ICME Evolution. Space Sci. Rev.212:1159-1219, DOI 10.1007/ s11214-017-0394-0

Manchester W IV (2001) The Role of Nonlinear Alfvén Waves in Shear Formation during Solar Magnetic Flux Emergence. ApJ547:503-519, DOI 10.1086/318342

Manchester W IV, Gombosi T, DeZeeuw D, Fan Y (2004) Eruption of a Buoyantly Emerging Magnetic Flux Rope. ApJ610:588-596, DOI 10.1086/421516

Martin SF (1998) Conditions for the Formation and Maintenance of Filaments (Invited Review). Sol. Phys.182:107-137, DOI 10.1023/A:1005026814076

Martin SF, Livi SHB, Wang J (1985) The cancellation of magnetic flux. II - In a decaying active region. Australian Journal of Physics 38:929-959, DOI 10.1071/PH850929

Martin SF, Panasenco O, Berger MA, Engvold O, Lin Y, Pevtsov AA, Srivastava N (2012) The Build-Up to Eruptive Solar Events Viewed as the Development of Chiral Systems. In: Rimmele TR, Tritschler A, Wöger F, Collados Vera M, Socas-Navarro H, Schlichenmaier R, Carlsson M, Berger T, Cadavid A, Gilbert PR, Goode PR, Knölker M (eds) Second ATST-EAST Meeting: Magnetic Fields from the Photosphere to the Corona., Astronomical Society of the Pacific Conference Series, vol 463, p 157, 1212.3646

Martínez-Sykora J, Hansteen V, Carlsson M (2008) Twisted Flux Tube Emergence From the Convection Zone to the Corona. ApJ679(1):871-888, DOI 10.1086/587028, 0712.3854

Mikic Z, Linker JA (1994) Disruption of coronal magnetic field arcades. ApJ430:898-912, DOI $10.1086 / 174460$

Mitra PK, Joshi B, Prasad A, Veronig AM, Bhattacharyya R (2018) Successive Flux Rope Eruptions from $\delta$-sunspots Region of NOAA 12673 and Associated X-class Eruptive Flares on 2017 September 6. ApJ869(1):69, DOI 10.3847/1538-4357/aaed26, 1810.13146

Moore RL, Roumeliotis G (1992) Triggering of Eruptive Flares - Destabilization of the Preflare Magnetic Field Configuration, vol 399, Springer, p 69. DOI 10.1007/3-540-55246-4_79

Moore RL, Hagyard MJ, Davis JM (1987) Flare research with the NASA/MSFC vector magnetograph: Observed characteristics of sheared magnetic fields that produce flares. Sol. Phys.113(1-2):347-352, DOI 10.1007/BF00147721

Moore RL, Sterling AC, Hudson HS, Lemen JR (2001) Onset of the Magnetic Explosion in Solar Flares and Coronal Mass Ejections. ApJ552:833-848, DOI 10.1086/320559

Moreno-Insertis F (2007) Three-dimensional numerical experiments of flux emergence into the corona. In: Shibata K, Nagata S, Sakurai T (eds) New Solar Physics with Solar-B Mission, Astronomical Society of the Pacific Conference Series, vol 369, p 335

Moreno-Insertis F, Galsgaard K (2013) Plasma Jets and Eruptions in Solar Coronal Holes: A Three-dimensional Flux Emergence Experiment. ApJ771:20, DOI 10.1088/0004-637X/771/ $1 / 20$

Moreno-Insertis F, Martinez-Sykora J, Hansteen VH, Muñoz D (2018) Small-scale Magnetic Flux Emergence in the Quiet Sun. ApJL859(2):L26, DOI 10.3847/2041-8213/aac648, 1806. 00489

Müller D, St Cyr OC, Zouganelis I, Gilbert HR, Marsden R, Nieves-Chinchilla T, Antonucci E, Auchère F, Berghmans D, Horbury TS, Howard RA, Krucker S, Maksimovic M, Owen CJ, Rochus P, Rodriguez-Pacheco J, Romoli M, Solanki SK, Bruno R, Carlsson M, Fludra A, Harra L, Hassler DM, Livi S, Louarn P, Peter H, Schühle U, Teriaca L, del Toro Iniesta JC, Wimmer-Schweingruber RF, Marsch E, Velli M, De Groof A, Walsh A, Williams D (2020) The Solar Orbiter mission. Science overview. A\&A642:A1, DOI 10.1051/0004-6361/ 202038467, 2009.00861

Murray MJ, Hood AW, Moreno-Insertis F, Galsgaard K, Archontis V (2006) 3D simulations identifying the effects of varying the twist and field strength of an emerging flux tube. A\&A460:909-923, DOI 10.1051/0004-6361:20065950

Nieves-Chinchilla T, Vourlidas A, Raymond JC, Linton MG, Al-haddad N, Savani NP, Szabo A, Hidalgo MA (2018) Understanding the Internal Magnetic Field Configurations of ICMEs Using More than 20 Years of Wind Observations. Sol. Phys.293:25, DOI $10.1007 / \mathrm{s} 11207-018-1247-\mathrm{z}$

Nindos A, Andrews MD (2004) The Association of Big Flares and Coronal Mass Ejections: What Is the Role of Magnetic Helicity? ApJL616:L175-L178, DOI 10.1086/426861

Nindos A, Patsourakos S, Wiegelmann T (2012) On the Role of the Background Overlying Magnetic Field in Solar Eruptions. ApJL748:L6, DOI 10.1088/2041-8205/748/1/L6

Nindos A, Patsourakos S, Vourlidas A, Tagikas C (2015) How Common Are Hot Magnetic Flux 
Ropes in the Low Solar Corona? A Statistical Study of EUV Observations. ApJ808:117, DOI $10.1088 / 0004-637 \mathrm{X} / 808 / 2 / 117,1507.03766$

Nóbrega-Siverio D, Moreno-Insertis F, Martínez-Sykora J (2018) On the Importance of the Nonequilibrium Ionization of Si IV and O IV and the Line of Sight in Solar Surges. ApJ858(1):8, DOI 10.3847/1538-4357/aab9b9, 1803.10251

Nordlund A, Stein RF, Asplund M (2009) Solar Surface Convection. Living Reviews in Solar Physics 6(1):2, DOI 10.12942/lrsp-2009-2

Okamoto TJ, Tsuneta S, Berger TE, Ichimoto K, Katsukawa Y, Lites BW, Nagata S, Shibata K, Shimizu T, Shine RA, Suematsu Y, Tarbell TD, Title AM (2007) Coronal Transverse Magnetohydrodynamic Waves in a Solar Prominence. Science 318(5856):1577, DOI 10.1126/ scien.1145447, 0801.1958

Okamoto TJ, Tsuneta S, Lites BW, Kubo M, Yokoyama T, Berger TE, Ichimoto K, Katsukawa Y, Nagata S, Shibata K, Shimizu T, Shine RA, Suematsu Y, Tarbell TD, Title AM (2008) Emergence of a Helical Flux Rope under an Active Region Prominence. ApJL673:L215, DOI 10.1086/528792, 0801.1956

Ouyang Y, Zhou YH, Chen PF, Fang C (2017) Chirality and Magnetic Configurations of Solar Filaments. ApJ835:94, DOI 10.3847/1538-4357/835/1/94, 1612.01054

Pariat E, Aulanier G, Schmieder B, Georgoulis MK, Rust DM, Bernasconi PN (2004) Resistive Emergence of Undulatory Flux Tubes. ApJ614(2):1099-1112, DOI 10.1086/423891

Pariat E, Leake JE, Valori G, Linton MG, Zuccarello FP, Dalmasse K (2017) Relative magnetic helicity as a diagnostic of solar eruptivity. A\&A601:A125, DOI 10.1051/0004-6361/ 201630043, 1703.10562

Park SH, Lee J, Choe GS, Chae J, Jeong H, Yang G, Jing J, Wang H (2008) The Variation of Relative Magnetic Helicity around Major Flares. ApJ686:1397-1403, DOI 10.1086/591117, 1004.2856

Park Sh, Chae J, Wang H (2010) Productivity of Solar Flares and Magnetic Helicity Injection in Active Regions. ApJ718:43-51, DOI 10.1088/0004-637X/718/1/43, 1005.3416

Parker EN (1983) Magnetic Neutral Sheets in Evolving Fields - Part Two - Formation of the Solar Corona. ApJ264:642, DOI 10.1086/160637

Patsourakos S, Vourlidas A, Kliem B (2010) Toward understanding the early stages of an impulsively accelerated coronal mass ejection. SECCHI observations. A\&A522:A100, DOI 10.1051/0004-6361/200913599, 1008.1171

Patsourakos S, Vourlidas A, Stenborg G (2013) Direct Evidence for a Fast Coronal Mass Ejection Driven by the Prior Formation and Subsequent Destabilization of a Magnetic Flux Rope. ApJ764:125, DOI 10.1088/0004-637X/764/2/125, 1211.7211

Pevtsov AA (2002) Active-Region Filaments and X-ray Sigmoids. Sol. Phys.207:111-123, DOI 10.1023/A:1015589802234

Pevtsov AA, Canfield RC, Metcalf TR (1995) Latitudinal Variation of Helicity of Photospheric Magnetic Fields. ApJL440:L109, DOI 10.1086/187773

Pevtsov AA, Berger MA, Nindos A, Norton AA, van Driel-Gesztelyi L (2014) Magnetic Helicity, Tilt, and Twist. Space Sci. Rev.186:285-324, DOI 10.1007/s11214-014-0082-2

Price DJ, Pomoell J, Lumme E, Kilpua EKJ (2019) Time-dependent data-driven coronal simulations of AR 12673 from emergence to eruption. A\&A628:A114, DOI 10.1051/0004-6361/ 201935535

Priest E (2014) Magnetohydrodynamics of the Sun. Cambridge University Press, DOI 10.1017/ CBO9781139020732

Priest ER, Longcope DW (2020) The Creation of Twist by Reconnection of Flux Tubes. Sol. Phys.295(3):48, DOI 10.1007/s11207-020-01608-0

Priest ER, Hood AW, Anzer U (1989) A twisted flux-tube model for solar prominences. I General properties. ApJ344:1010-1025, DOI 10.1086/167868

Priest ER, Longcope DW, Janvier M (2016) Evolution of magnetic helicity during eruptive flares and coronal mass ejections. Sol. Phys.291:2017-2036, DOI 10.1007/s11207-016-0962-6, 1607.03874

Rachmeler LA, Gibson SE, Dove JB, DeVore CR, Fan Y (2013) Polarimetric Properties of Flux Ropes and Sheared Arcades in Coronal Prominence Cavities. Sol. Phys.288:617-636, DOI 10.1007/s11207-013-0325-5, 1304.7594

Rempel M, Cheung MCM (2014) Numerical Simulations of Active Region Scale Flux Emergence: From Spot Formation to Decay. ApJ785(2):90, DOI 10.1088/0004-637X/785/2/90, 1402.4703

Roumeliotis G, Sturrock PA, Antiochos SK (1994) A Numerical Study of the Sudden Eruption 
of Sheared Magnetic Fields. ApJ423:847, DOI 10.1086/173862

Rust DM, LaBonte BJ (2005) Observational Evidence of the Kink Instability in Solar Filament Eruptions and Sigmoids. ApJL622:L69-L72, DOI 10.1086/429379

Savcheva A, van Ballegooijen A (2009) Nonlinear Force-free Modeling of a Long-lasting Coronal Sigmoid. ApJ703:1766-1777, DOI 10.1088/0004-637X/703/2/1766

Savcheva AS, Green LM, van Ballegooijen AA, DeLuca EE (2012) Photospheric Flux Cancellation and the Build-up of Sigmoidal Flux Ropes on the Sun. ApJ759:105, DOI 10.1088/0004-637X/759/2/105

Savcheva AS, McKillop SC, McCauley PI, Hanson EM, DeLuca EE (2014) A New Sigmoid Catalog from Hinode and the Solar Dynamics Observatory: Statistical Properties and Evolutionary Histories. Sol. Phys.289:3297-3311, DOI 10.1007/s11207-013-0469-3

Schmieder B, Raadu MA, Wiik JE (1991) Fine structure of solar filaments. II - Dynamics of threads and footpoints. A\&A252:353-365

Schmieder B, Démoulin P, Aulanier G, Golub L (1996) Differential Magnetic Field Shear in an Active Region. ApJ467:881, DOI 10.1086/177662

Schmieder B, Zapiór M, López Ariste A, Levens P, Labrosse N, Gravet R (2017) Reconstruction of a helical prominence in 3D from IRIS spectra and images. A\&A606:A30, DOI 10.1051/ 0004-6361/201730839, 1706.08078

Schrijver CJ (2007) A Characteristic Magnetic Field Pattern Associated with All Major Solar Flares and Its Use in Flare Forecasting. ApJL655:L117-L120, DOI 10.1086/511857

Schrijver CJ, Title AM, Berger TE, Fletcher L, Hurlburt NE, Nightingale RW, Shine RA, Tarbell TD, Wolfson J, Golub L, Bookbinder JA, Deluca EE, McMullen RA, Warren HP, Kankelborg CC, Handy BN, de Pontieu B (1999) A new view of the solar outer atmosphere by the Transition Region and Coronal Explorer. Sol. Phys.187:261-302, DOI 10.1023/A: 1005194519642

Schrijver CJ, DeRosa ML, Metcalf T, Barnes G, Lites B, Tarbell T, McTiernan J, Valori G, Wiegelmann T, Wheatland MS, Amari T, Aulanier G, Démoulin P, Fuhrmann M, Kusano K, Régnier S, Thalmann JK (2008) Nonlinear Force-free Field Modeling of a Solar Active Region around the Time of a Major Flare and Coronal Mass Ejection. ApJ675:1637-1644, DOI 10.1086/527413, 0712.0023

Seehafer N (1990) Electric Current Helicity in the Solar Atmosphere. Sol. Phys.125(2):219-232, DOI 10.1007/BF00158402

Stein RF (2012) Solar Surface Magneto-Convection. Living Reviews in Solar Physics 9(1):4, DOI 10.12942/lrsp-2012-4

Stein RF, Nordlund A (2006) Solar Small-Scale Magnetoconvection. ApJ642:1246-1255, DOI $10.1086 / 501445$

Stein RF, Nordlund $\AA$ (2012) On the Formation of Active Regions. ApJL753:L13, DOI 10. $1088 / 2041-8205 / 753 / 1 /$ L13

Stein RF, Lagerfjärd A, Nordlund Å, Georgobiani D (2011) Solar Flux Emergence Simulations. Sol. Phys.268:271-282, DOI 10.1007/s11207-010-9510-y

Sterling AC, Moore RL (2001a) Eit crinkles as evidence for the breakout model of solar eruptions. ApJ560:1045-1057, DOI 10.1086/322241

Sterling AC, Moore RL (2001b) Internal and external reconnection in a series of homologous solar flares. JGR106:25,227-25,238, DOI 10.1029/2000JA004001

Sterling AC, Moore RL (2004) Evidence for gradual external reconnection before explosive eruption of a solar filament. ApJ602:1024-1036, DOI 10.1086/379763

Sterling AC, Moore RL, Thompson BJ (2001) Eit and sxt observations of a quiet-region filament ejection: First eruption, then reconnection. ApJL561:L219-L222, DOI 10.1086/324765

Sturrock Z, Hood AW (2016) Sunspot rotation. II. Effects of varying the field strength and twist of an emerging flux tube. A\&A593:A63, DOI 10.1051/0004-6361/201628360

Sturrock Z, Hood AW, Archontis V, McNeill CM (2015) Sunspot rotation. I. A consequence of flux emergence. A\&A582:A76, DOI 10.1051/0004-6361/201526521

Su Y, van Ballegooijen A, Schmieder B, Berlicki A, Guo Y, Golub L, Huang G (2009) Flare Energy Build-up in a Decaying Active Region Near a Coronal Hole. ApJ704:341-353, DOI 10.1088/0004-637X/704/1/341

Su Y, Surges V, van Ballegooijen A, DeLuca E, Golub L (2011) Observations and Magnetic Field Modeling of the Flare/coronal Mass Ejection Event on 2010 April 8. ApJ734(1):53, DOI 10.1088/0004-637X/734/1/53

Sudol JJ, Harvey JW (2005) Longitudinal Magnetic Field Changes Accompanying Solar Flares. The Astrophysical Journal 635:647-658, DOI 10.1086/497361, URL http://adsabs. 
harvard.edu/abs/2005ApJ ..635. .647S

Sun X, Hoeksema JT, Liu Y, Wiegelmann T, Hayashi K, Chen Q, Thalmann J (2012) Evolution of Magnetic Field and Energy in a Major Eruptive Active Region Based on SDO/HMI Observation. ApJ748:77, DOI 10.1088/0004-637X/748/2/77, 1201.3404

Syntelis P, Archontis V, Gontikakis C, Tsinganos K (2015) Emergence of non-twisted magnetic fields in the Sun: Jets and atmospheric response. A\&A584:A10, DOI 10.1051/0004-6361/ 201423781

Syntelis P, Archontis V, Tsinganos K (2017) Recurrent CME-like Eruptions in Emerging Flux Regions. I. On the Mechanism of Eruptions. ApJ850:95, DOI 10.3847/1538-4357/aa9612

Syntelis P, Archontis V, Hood A (2019a) Successful and Failed Flux Tube Emergence in the Solar Interior. ApJ874:15, DOI 10.3847/1538-4357/ab0959, 1902.07969

Syntelis P, Archontis V, Tsinganos K (2019b) Recurrent CME-like Eruptions in Emerging Flux Regions. II. Scaling of Energy and Collision of Successive Eruptions. ApJ876(1):61, DOI $10.3847 / 1538-4357 / \mathrm{ab} 16 \mathrm{~d} 2,1904.03923$

Syntelis P, Lee EJ, Fairbairn CW, Archontis V, Hood AW (2019c) Eruptions and flaring activity in emerging quadrupolar regions. A\&A630:A134, DOI 10.1051/0004-6361/201936246, 1909.01446

Takasao S, Fan Y, Cheung MCM, Shibata K (2015) Numerical Study on the Emergence of Kinked Flux Tube for Understanding of Possible Origin of $\delta$-spot Regions. ApJ813(2):112, DOI 10.1088/0004-637X/813/2/112, 1511.02863

Takizawa K, Kitai R, Zhang Y (2012) Prominent Photospheric Downflows on Magnetic Neutral Line in a Delta-Type Sunspot. Sol. Phys.281:599-609, DOI 10.1007/s11207-012-0116-4

Tanaka K (1991) Studies on a very flare-active delta group - Peculiar delta SPOT evolution and inferred subsurface magnetic rope structure. Sol. Phys.136:133-149, DOI $10.1007 / \mathrm{BF} 00151700$

Temmer M, Veronig AM, Kontar EP, Krucker S, Vršnak B (2010) Combined STEREO/RHESSI Study of Coronal Mass Ejection Acceleration and Particle Acceleration in Solar Flares. ApJ712(2):1410-1420, DOI 10.1088/0004-637X/712/2/1410, 1002.3080

Titov VS, Priest ER, Démoulin P (1993) Conditions for the appearance of "bald patches" at the solar surface. A\&A276:564

Titov VS, Hornig G, Démoulin P (2002) Theory of magnetic connectivity in the solar corona. J Geophys Res 107:1164

Titov VS, Török T, Mikic Z, Linker JA (2014) A Method for Embedding Circular Force-free Flux Ropes in Potential Magnetic Fields. ApJ790(2):163, DOI 10.1088/0004-637X/790/2/ 163

Titov VS, Mikić Z, Török T, Linker JA, Panasenco O (2017) 2010 August 1-2 Sympathetic Eruptions. II. Magnetic Topology of the MHD Background Field. ApJ845:141, DOI 10. 3847/1538-4357/aa81ce, 1707.07773

Titov VS, Downs C, Mikić Z, Török T, Linker JA, Caplan RM (2018) Regularized Biot-Savart Laws for Modeling Magnetic Flux Ropes. ApJL852:L21, DOI 10.3847/2041-8213/aaa3da, 1712.06708

Tomczyk S, Landi E, Burkepile JT, Casini R, DeLuca EE, Fan Y, Gibson SE, Lin H, McIntosh SW, Solomon SC, Toma G, Wijn AG, Zhang J (2016) Scientific objectives and capabilities of the Coronal Solar Magnetism Observatory. Journal of Geophysical Research (Space Physics) 121:7470-7487, DOI 10.1002/2016JA022871

Toriumi S (2014) Observations and modeling of the solar flux emergence. PASJ66:S6, DOI $10.1093 / \mathrm{pasj} / \mathrm{psu} 100$

Toriumi S, Hotta H (2019) Spontaneous Generation of $\delta$-sunspots in Convective Magnetohydrodynamic Simulation of Magnetic Flux Emergence. ApJL886(1):L21, DOI 10.3847/ 2041-8213/ab55e7, 1911.03909

Toriumi S, Takasao S (2017) Numerical Simulations of Flare-productive Active Regions: $\delta$ sunspots, Sheared Polarity Inversion Lines, Energy Storage, and Predictions. ApJ850:39, DOI $10.3847 / 1538-4357 /$ aa95c2

Toriumi S, Wang H (2019) Flare-productive active regions. Living Reviews in Solar Physics 16(1):3, DOI 10.1007/s41116-019-0019-7, 1904.12027

Toriumi S, Yokoyama T (2010) Two-step Emergence of the Magnetic Flux Sheet from the Solar Convection Zone. ApJ714:505-516, DOI 10.1088/0004-637X/714/1/505

Toriumi S, Iida Y, Kusano K, Bamba Y, Imada S (2014) Formation of a flare-productive active region: Observation and numerical simulation of noaa ar 11158. Sol. Phys.289:3351-3369, DOI 10.1007/s11207-014-0502-1, 1403.4029 
Török T, Kliem B (2003) The evolution of twisting coronal magnetic flux tubes. A\&A406:10431059, DOI 10.1051/0004-6361:20030692

Török T, Kliem B (2005) Confined and Ejective Eruptions of Kink-unstable Flux Ropes. Astrophysical Journal Letters 630:L97-L100, DOI 10.1086/462412, arXiv:astro-ph/0507662

Török T, Kliem B (2007) Numerical simulations of fast and slow coronal mass ejections. Astronomische Nachrichten 328:743, DOI 10.1002/asna.200710795, 0705.2100

Török T, Kliem B, Titov VS (2004) Ideal kink instability of a magnetic loop equilibrium. Astronomy and Astrophysics 413:L27-L30, DOI 10.1051/0004-6361:20031691, astro-ph/ 0311198

Török T, Berger MA, Kliem B (2010) The writhe of helical structures in the solar corona. A\&A516:A49, DOI 10.1051/0004-6361/200913578, 1004.3918

Török T, Kliem B, Berger MA, Linton MG, Démoulin P, van Driel-Gesztelyi L (2014a) The evolution of writhe in kink-unstable flux ropes and erupting filaments. Plasma Physics and Controlled Fusion 56(6):064012, DOI 10.1088/0741-3335/56/6/064012, 1403.1565

Török T, Leake JE, Titov VS, Archontis V, Mikić Z, Linton MG, Dalmasse K, Aulanier G, Kliem B (2014b) Distribution of Electric Currents in Solar Active Regions. ApJL782:L10, DOI 10.1088/2041-8205/782/1/L10, 1401.2931

Török T, Downs C, Linker JA, Lionello R, Titov VS, Mikić Z, Riley P, Caplan RM, Wijaya J (2018a) Sun-to-Earth MHD Simulation of the 2000 July 14 Bastille Day Eruption. ApJ856:75, DOI 10.3847/1538-4357/aab36d, 1801.05903

Török T, Leake JE, Titov VS, Sun X, Linton M, Mikic Z, Lionello R, Moore RL, Sterling AC (2018b) Conjoined Flux Ropes: A New Mechanism for Filament-Channel Formation at External Polarity Inversion Lines. In: AGU Fall Meeting Abstracts, vol 2018, pp SH12A-04

Tschernitz J, Veronig AM, Thalmann JK, Hinterreiter J, Pötzi W (2018) Reconnection Fluxes in Eruptive and Confined Flares and Implications for Superflares on the Sun. ApJ853(1):41, DOI 10.3847/1538-4357/aaa199, 1712.04701

Tziotziou K, Georgoulis MK, Raouafi NE (2012) The Magnetic Energy-Helicity Diagram of Solar Active Regions. ApJL759:L4, DOI 10.1088/2041-8205/759/1/L4, 1209.5612

Tziotziou K, Georgoulis MK, Liu Y (2013) Interpreting Eruptive Behavior in NOAA AR 11158 via the Region's Magnetic Energy and Relative-helicity Budgets. ApJ772:115, DOI 10.1088/0004-637X/772/2/115, 1306.2135

van Ballegooijen AA (2004) Observations and Modeling of a Filament on the Sun. ApJ612:519529 , DOI $10.1086 / 422512$

van Ballegooijen AA, Martens PCH (1989) Formation and eruption of solar prominences. Astrophysical Journal 343:971-984, DOI 10.1086/167766

van Ballegooijen AA, Priest ER, Mackay DH (2000) Mean Field Model for the Formation of Filament Channels on the Sun. Astrophysical Journal 539:983-994, DOI 10.1086/309265

van Driel-Gesztelyi L, Green LM (2015) Evolution of Active Regions. Living Reviews in Solar Physics 12(1):1, DOI 10.1007/lrsp-2015-1

van Driel-Gesztelyi L, Malherbe JM, Démoulin P (2000) Emergence of a U-loop - subphotospheric link between solar active regions. A\&A364:845-852

Vargas Domínguez S, MacTaggart D, Green L, van Driel-Gesztelyi L, Hood AW (2012) On Signatures of Twisted Magnetic Flux Tube Emergence. Sol. Phys.278:33-45, DOI 10.1007/ s11207-011-9789-3, 1105.0758

Veronig AM, Polanec W (2015) Magnetic Reconnection Rates and Energy Release in a Confined X-class Flare. Sol. Phys.290:2923-2942, DOI 10.1007/s11207-015-0789-6, 1509.07089

Veronig AM, Podladchikova T, Dissauer K, Temmer M, Seaton DB, Long D, Guo J, Vršnak B, Harra L, Kliem B (2018) Genesis and Impulsive Evolution of the 2017 September 10 Coronal Mass Ejection. ApJ868:107, DOI 10.3847/1538-4357/aaeac5, 1810.09320

Vourlidas A (2014) The flux rope nature of coronal mass ejections. Plasma Phys Control Fusion 56(6):064,001, DOI 10.1088/0741-3335/56/6/064001, URL http://iopscience.iop. org/0741-3335/56/6/064001

Vourlidas A (2015) Mission to the Sun-Earth L5 Lagrangian Point: An Optimal Platform for Space Weather Research. Space Weather 13:197-201, DOI 10.1002/2015SW001173

Vourlidas A, Howard RA, Morrill JS, Munz S (2002) Analysis of Lasco Observations of Streamer Blowout Events. In: Wang H, Xu R (eds) Solar-Terrestrial Magnetic Activity and Space Environment, vol 14, p 201

Vourlidas A, Sanchez Andrade-Nuño B, Landi E, Patsourakos S, Teriaca L, Schühle U, Korendyke CM, Nestoras I (2010) The Structure and Dynamics of the Upper Chromosphere and Lower Transition Region as Revealed by the Subarcsecond VAULT Observations. Sol. 
Phys.261(1):53-75, DOI 10.1007/s11207-009-9475-x, 0912.2272

Vourlidas A, Lynch BJ, Howard RA, Li Y (2013) How Many CMEs Have Flux Ropes? Deciphering the Signatures of Shocks, Flux Ropes, and Prominences in Coronagraph Observations of CMEs. Sol. Phys.284:179-201, DOI 10.1007/s11207-012-0084-8, 1207.1599

Vourlidas A, Balmaceda LA, Stenborg G, Dal Lago A (2017) Multi-viewpoint Coronal Mass Ejection Catalog Based on STEREO COR2 Observations. ApJ838:141, DOI 10.3847/ $1538-4357 / \mathrm{aa} 67 \mathrm{f0}$

Vourlidas A, Patsourakos S, Savani NP (2019) Predicting the geoeffective properties of coronal mass ejections: current status, open issues and path forward. Philosophical Transactions of the Royal Society A: Mathematical, Physical and Engineering Sciences 377(2148):20180,096, DOI 10.1098/rsta.2018.0096, URL http://www. royalsocietypublishing.org/doi/10.1098/rsta.2018.0096

Vrsnak B (1990) Dynamics and Internal Structure of an Eruptive Prominence. Sol. Phys.127(1):129-137, DOI 10.1007/BF00158518

Wang H, Cao W, Liu C, Xu Y, Liu R, Zeng Z, Chae J, Ji H (2015) Witnessing magnetic twist with high-resolution observation from the 1.6-m New Solar Telescope. Nature Communications 6:7008, DOI 10.1038/ncomms8008

Wang YM, Muglach K (2007) On the Formation of Filament Channels. ApJ666:1284-1295, DOI 10.1086/520623

Wang YM, Muglach K (2013) Transient Brightenings Associated with Flux Cancellation along a Filament Channel. ApJ763:97, DOI 10.1088/0004-637X/763/2/97

Wang YM, Stenborg G (2010) Spinning Motions in Coronal Cavities. ApJL719:L181-L184, DOI $10.1088 / 2041-8205 / 719 / 2 / L 181$

Wiegelmann T (2008) Nonlinear force-free modeling of the solar coronal magnetic field. Journal of Geophysical Research (Space Physics) 113:A03S02, DOI 10.1029/2007JA012432, 0801. 2902

Wiegelmann T, Thalmann JK, Solanki SK (2014) The magnetic field in the solar atmosphere. A\&A Rev.22:78, DOI 10.1007/s00159-014-0078-7, 1410.4214

Woods MM, Inoue S, Harra LK, Matthews SA, Kusano K (2020) Serial Flaring in an Active Region: Exploring Why Only One Flare Is Eruptive. ApJ890(1):84, DOI 10.3847/1538-4357/ ab6bc8

Wright AN (2019) Partitioning of Magnetic Helicity in Reconnected Flux Tubes. ApJ878(2):102, DOI 10.3847/1538-4357/ab2120

Wu ST, Bao JJ, An CH, Tandberg-Hanssen E (1990) The Role of Condensation and Heat Conduction in the Formation of Prominences - a Magnetohydrodynamic Simulation. Sol. Phys.125(2):277-293, DOI 10.1007/BF00158407

Wyper PF, Pontin DI (2014a) Dynamic topology and flux rope evolution during non-linear tearing of 3D null point current sheets. Physics of Plasmas 21(10):102102, DOI 10.1063/1. 4896060, 1406.6120

Wyper PF, Pontin DI (2014b) Non-linear tearing of 3D null point current sheets. Physics of Plasmas 21(8):082114, DOI 10.1063/1.4893149, 1406.1622

Xia C, Keppens R (2016) Formation and Plasma Circulation of Solar Prominences. ApJ823:22, DOI 10.3847/0004-637X/823/1/22, 1603.05397

Xia C, Keppens R, Antolin P, Porth O (2014) Simulating the in Situ Condensation Process of Solar Prominences. ApJL792:L38, DOI 10.1088/2041-8205/792/2/L38, 1408.4249

Xu Z, Lagg A, Solanki S, Liu Y (2012) Magnetic Fields of an Active Region Filament from Full Stokes Analysis of Si I $1082.7 \mathrm{~nm}$ and He I 1083.0 nm. ApJ749:138, DOI 10.1088/ 0004-637X/749/2/138, 1202.4562

Xue Z, Yan X, Cheng X, Yang L, Su Y, Kliem B, Zhang J, Liu Z, Bi Y, Xiang Y, Yang K, Zhao L (2016) Observing the release of twist by magnetic reconnection in a solar filament eruption. Nature Communications 7:11837, DOI 10.1038/ncomms11837

Yang S, Zhang J, Borrero JM (2009) Dipolar Evolution in a Coronal Hole Region. ApJ703:1012-1020, DOI 10.1088/0004-637X/703/1/1012, 0908.0578

Yang WH, Sturrock PA, Antiochos SK (1986) Force-free magnetic fields - The magnetofrictional method. ApJ309:383-391, DOI 10.1086/164610

Yardley SL, Green LM, Williams DR, van Driel-Gesztelyi L, Valori G, Dacie S (2016) Flux Cancellation and the Evolution of the Eruptive Filament of 2011 June 7 . ApJ827:151, DOI 10.3847/0004-637X/827/2/151, 1606.08264

Yardley SL, Green LM, van Driel-Gesztelyi L, Williams DR, Mackay DH (2018a) The Role of Flux Cancellation in Eruptions from Bipolar ARs. ApJ866:8, DOI 10.3847/1538-4357/ 
aade4a, 1808.10635

Yardley SL, Mackay DH, Green LM (2018b) Simulating the Coronal Evolution of AR 11437 Using SDO/HMI Magnetograms. ApJ852(2):82, DOI 10.3847/1538-4357/aa9f20, 1712.00396

Yeates AR, Mackay DH (2009) Initiation of Coronal Mass Ejections in a Global Evolution Model. ApJ699(2):1024-1037, DOI 10.1088/0004-637X/699/2/1024, 0904.4419

Yeates AR, Mackay DH (2012) Chirality of High-latitude Filaments over Solar Cycle 23. ApJL753:L34, DOI 10.1088/2041-8205/753/2/L34, 1206.2327

Yeates AR, Mackay DH, van Ballegooijen AA (2008) Evolution and Distribution of Current Helicity in Full-Sun Simulations. Astrophysical Journal Letters 680:L165, DOI 10.1086/ 590057, 0805.1883

Yelles Chaouche L, Kuckein C, Martínez Pillet V, Moreno-Insertis F (2012) The Threedimensional Structure of an Active Region Filament as Extrapolated from Photospheric and Chromospheric Observations. ApJ748:23, DOI 10.1088/0004-637X/748/1/23, 1201.2456

Zhang H, Brandenburg A, Sokoloff DD (2014) Magnetic Helicity and Energy Spectra of a Solar Active Region. ApJL784:L45, DOI 10.1088/2041-8205/784/2/L45, 1311.2432

Zhang H, Brandenburg A, Sokoloff DD (2016) Evolution of Magnetic Helicity and Energy Spectra of Solar Active Regions. ApJ819:146, DOI 10.3847/0004-637X/819/2/146, 1503. 00846

Zhang J, Dere KP (2006) A Statistical Study of Main and Residual Accelerations of Coronal Mass Ejections. ApJ649(2):1100-1109, DOI 10.1086/506903

Zhang J, Dere KP, Howard RA, Vourlidas A (2004) A Study of the Kinematic Evolution of Coronal Mass Ejections. ApJ604(1):420-432, DOI 10.1086/381725

Zhang J, Cheng X, Ding MD (2012) Observation of an evolving magnetic flux rope before and during a solar eruption. Nature Communications 3:747, DOI 10.1038/ncomms1753, 1203.4859

Zhao J, Gilchrist SA, Aulanier G, Schmieder B, Pariat E, Li H (2016) Hooked Flare Ribbons and Flux-rope-related QSL Footprints. ApJ823:62, DOI 10.3847/0004-637X/823/1/62, 1603.07563

Zhao L, DeVore CR, Antiochos SK, Zurbuchen TH (2015) Numerical Simulations of Helicity Condensation in the Solar Corona. ApJ805:61, DOI 10.1088/0004-637X/805/1/61

Zhou YH, Xia C, Keppens R, Fang C, Chen PF (2018) Three-dimensional MHD Simulations of Solar Prominence Oscillations in a Magnetic Flux Rope. ApJ856(2):179, DOI 10.3847/ $1538-4357 /$ aab614, 1803.03385

Zhou Z, Zhang J, Wang Y, Liu R, Chintzoglou G (2017) Toward Understanding the 3D Structure and Evolution of Magnetic Flux Ropes in an Extremely Long Duration Eruptive Flare. ApJ851:133, DOI 10.3847/1538-4357/aa9bd9

Zhu C, Qiu J, Liewer P, Vourlidas A, Spiegel M, Hu Q (2020) How Does Magnetic Reconnection Drive the Early-stage Evolution of Coronal Mass Ejections? ApJ893(2):141, DOI 10.3847/ 1538-4357/ab838a, 2003.11134

Zhu X, Wang H, Du Z, He H (2016) Forced Field Extrapolation of the Magnetic Structure of the $\mathrm{H} \alpha$ fibrils in the Solar Chromosphere. ApJ826(1):51, DOI 10.3847/0004-637X/826/1/51, 1604.00455

Zirin H, Liggett MA (1987) Delta spots and great flares. Sol. Phys.113:267-281, DOI 10.1007/ BF00147707

Zouganelis I, De Groof A, Walsh AP, Williams DR, Müller D, St Cyr OC, Auchère F, Berghmans D, Fludra A, Horbury TS, Howard RA, Krucker S, Maksimovic M, Owen CJ, Rodríguez-Pacheco J, Romoli M, Solanki SK, Watson C, Sanchez L, Lefort J, Osuna P, Gilbert HR, Nieves-Chinchilla T, Abbo L, Alexandrova O, Anastasiadis A, Andretta V, Antonucci E, Appourchaux T, Aran A, Arge CN, Aulanier G, Baker D, Bale SD, Battaglia M, Bellot Rubio L, Bemporad A, Berthomier M, Bocchialini K, Bonnin X, Brun AS, Bruno R, Buchlin E, Büchner J, Bucik R, Carcaboso F, Carr R, Carrasco-Blázquez I, Cecconi B, Cernuda Cangas I, Chen CHK, Chitta LP, Chust T, Dalmasse K, D'Amicis R, Da Deppo V, De Marco R, Dolei S, Dolla L, Dudok de Wit T, van Driel-Gesztelyi L, Eastwood JP, Espinosa Lara F, Etesi L, Fedorov A, Félix-Redondo F, Fineschi S, Fleck B, Fontaine D, Fox NJ, Gandorfer A, Génot V, Georgoulis MK, Gissot S, Giunta A, Gizon L, Gómez-Herrero R, Gontikakis C, Graham G, Green L, Grundy T, Haberreiter M, Harra LK, Hassler DM, Hirzberger J, Ho GC, Hurford G, Innes D, Issautier K, James AW, Janitzek N, Janvier M, Jeffrey N, Jenkins J, Khotyaintsev Y, Klein KL, Kontar EP, Kontogiannis I, Krafft C, Krasnoselskikh V, Kretzschmar M, Labrosse N, Lagg A, Landini F, Lavraud B, Leon I, Lepri ST, Lewis GR, Liewer P, Linker J, Livi S, Long DM, Louarn P, Malandraki O, Maloney 
S, Martinez-Pillet V, Martinovic M, Masson A, Matthews S, Matteini L, Meyer-Vernet N, Moraitis K, Morton RJ, Musset S, Nicolaou G, Nindos A, O'Brien H, Orozco Suarez D, Owens M, Pancrazzi M, Papaioannou A, Parenti S, Pariat E, Patsourakos S, Perrone D, Peter H, Pinto RF, Plainaki C, Plettemeier D, Plunkett SP, Raines JM, Raouafi N, Reid H, Retino A, Rezeau L, Rochus P, Rodriguez L, Rodriguez-Garcia L, Roth M, Rouillard AP, Sahraoui F, Sasso C, Schou J, Schühle U, Sorriso-Valvo L, Soucek J, Spadaro D, Stangalini M, Stansby D, Steller M, Strugarek A, Štverák Š, Susino R, Telloni D, Terasa C, Teriaca L, Toledo-Redondo S, del Toro Iniesta JC, Tsiropoula G, Tsounis A, Tziotziou K, Valentini F, Vaivads A, Vecchio A, Velli M, Verbeeck C, Verdini A, Verscharen D, Vilmer N, Vourlidas A, Wicks R, Wimmer-Schweingruber RF, Wiegelmann T, Young PR, Zhukov AN (2020) The Solar Orbiter Science Activity Plan. Translating solar and heliospheric physics questions into action. A\&A642:A3, DOI 10.1051/0004-6361/202038445, 2009.10772

Zuccarello FP, Aulanier G, Gilchrist SA (2015) Critical Decay Index at the Onset of Solar Eruptions. ApJ814:126, DOI 10.1088/0004-637X/814/2/126, 1510.03713

Zuccarello FP, Aulanier G, Gilchrist SA (2016) The Apparent Critical Decay Index at the Onset of Solar Prominence Eruptions. ApJL821(2):L23, DOI 10.3847/2041-8205/821/2/L23

Zwaan C (1987) Elements and patterns in the solar magnetic field. ARA\&A25:83-111, DOI 10.1146/annurev.aa.25.090187.000503 